\title{
Connecting the dots : functional networks and cognition in chronic epilepsy
}

Citation for published version (APA):

Vlooswijk, M. C. G. (2011). Connecting the dots : functional networks and cognition in chronic epilepsy. [Doctoral Thesis, Maastricht University]. Datawyse / Universitaire Pers Maastricht. https://doi.org/10.26481/dis.20110930mv

Document status and date:

Published: 01/01/2011

DOI:

10.26481/dis.20110930mv

Document Version:

Publisher's PDF, also known as Version of record

\section{Please check the document version of this publication:}

- A submitted manuscript is the version of the article upon submission and before peer-review. There can be important differences between the submitted version and the official published version of record.

People interested in the research are advised to contact the author for the final version of the publication, or visit the DOI to the publisher's website.

- The final author version and the galley proof are versions of the publication after peer review.

- The final published version features the final layout of the paper including the volume, issue and page numbers.

Link to publication

\footnotetext{
General rights rights.

- You may freely distribute the URL identifying the publication in the public portal. please follow below link for the End User Agreement:

www.umlib.nl/taverne-license

Take down policy

If you believe that this document breaches copyright please contact us at:

repository@maastrichtuniversity.nl

providing details and we will investigate your claim.
}

Copyright and moral rights for the publications made accessible in the public portal are retained by the authors and/or other copyright owners and it is a condition of accessing publications that users recognise and abide by the legal requirements associated with these

- Users may download and print one copy of any publication from the public portal for the purpose of private study or research.

- You may not further distribute the material or use it for any profit-making activity or commercial gain

If the publication is distributed under the terms of Article $25 \mathrm{fa}$ of the Dutch Copyright Act, indicated by the "Taverne" license above, 
Connecting the dots

Functional networks and cognition in chronic epilepsy 
(C) Mariëlle Vlooswijk, Maastricht 2011

Layout: Tiny Wouters

Cover design: Sophie Valkenier

Production: Datawyse/Universitaire Pers Maastricht

ISBN: 9789461590794

The printing of this thesis was financially supported by the Nationaal Epilepsie Fonds, Kempenhaeghe Sector for Research and Development, School for Mental Health and Neuroscience (MHeNS), Maastricht University Medical Center, UCB Pharma B.V. Breda and GlaxoSmithKline. 


\title{
Connecting the dots
}

\section{Functional networks and cognition in chronic epilepsy}

\author{
PROEFSCHRIFT
}

Ter verkrijging van de graad van doctor

aan de Universiteit Maastricht, op gezag van de Rector Magnificus,

Prof. Mr. G.P.M.F. Mols,

volgens het besluit van het College van Decanen,

in het openbaar te verdedigen

op vrijdag 30 september 2011 om 12.00 uur

door

Maria Cornelia Gerarda Vlooswijk

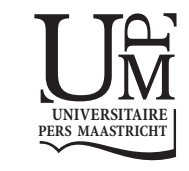




\section{Promotores}

Prof. Dr. A.P. Aldenkamp

Prof. Dr. J.E. Wildberger

\section{Co-promotores}

Dr. Ir. W.H. Backes

Dr. H.J.M. Majoie

\section{Beoordelingscommissie}

Prof. Dr. J.T. Wilmink (voorzitter)

Prof. Dr. P.A.J.M. Boon, Universiteit Gent (België)

Dr. R.J. van Oostenbrugge

Prof. Dr. J.S.H. Vles 


\section{Contents}

$\begin{array}{lll}\text { Chapter } 1 & \text { General introduction } & 7\end{array}$

Chapter 2 Functional MRI in chronic epilepsy: associations with 15 cognitive impairment

Chapter 3 Cognitive fMRI and neuropsychological assessment in patients with secondarily generalized seizures

Chapter 4 Hippocampal MRI volumetry at 3 Tesla: reliability and practical guidance

Chapter 5 Memory processes and prefrontal network dysfunction in cryptogenic epilepsy

Chapter 6 Functional connectivity and language impairment in cryptogenic localization-related epilepsy

Chapter 7 Loss of network efficiency associated with cognitive decline in chronic epilepsy

Chapter 8 General discussion

Summary

Samenvatting

Dankwoord

List of publications

Curriculum Vitae 



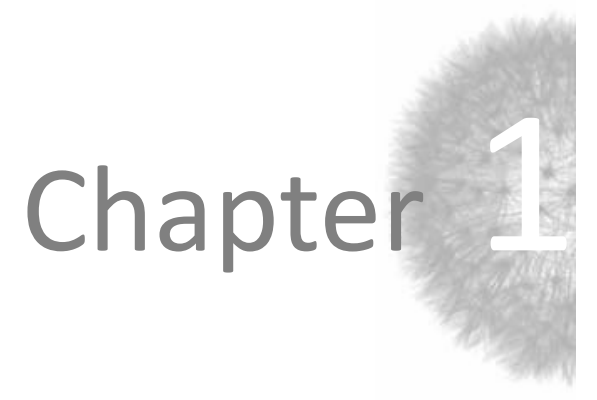

General introduction 
$\left.{ }_{8}\right|_{\text {chaserer } 1}$ 


\section{Epilepsy}

Epilepsy is a disorder characterized by the occurrence of unprovoked epileptic seizures. Epileptic seizures are the clinical manifestation of excessive and/or hypersynchronous activity of neurons in the brain. ${ }^{1}$ It is a heterogeneous disorder with a wide variety of clinical expressions and underlying etiologies. Epilepsy is therefore not a single disease entity, but encompasses a number of diseases. ${ }^{2}$ In the Netherlands, 100,000 to 120,000 people live with epilepsy. ${ }^{3}$ Around $70 \%$ of the people with epilepsy can become seizure-free with appropriate treatment, mostly antiepileptic drugs. In the remaining $30 \%$, seizures may persist despite antiepileptic drug use, epilepsy surgery and/or other treatments. Living with epilepsy can be a great burden for the patient himself and those who surround him. Since epileptic seizures mostly occur unexpectedly, they may cause physical harm, as well as psychological stress due to the unpredictability. Furthermore, patients may be confronted on a daily basis with driving and working restrictions, possibly adverse side-effects of antiepileptic drugs and psychological and cognitive complications.

The classification of epilepsy syndromes is roughly based on two characteristics: location of onset of the epileptic discharges, and underlying cause. ${ }^{4}$ The onset of epileptic discharges may be generalized - both cerebral hemispheres are rapidly involved at onset of the epileptic seizure - or localization-related with onset in a circumscribed cerebral region (with or without spread throughout the rest of the brain). The underlying causes can be generally divided into symptomatic - due to a known underlying cause, mostly a structural abnormality - , idiopathic - no known underlying cause, probably in part a genetic predisposition - and cryptogenic. This last category is based on the fact that a symptomatic lesion is suspected, but cannot be identified with current imaging techniques.

\section{Epilepsy and cognition}

\section{Historical perspective}

It has long been recognized that epilepsy is of great influence on the mental and cognitive status of the patients who suffer from it. Some have described an association of genius and epilepsy, with examples of great world leaders with epilepsy such as Alexander the Great, and Napoleon Bonaparte, and famous writers such as Charles Dickens and Fyodor Dostoevsky. On the other hand, examples of a more negative effect of epilepsy on the mental and cognitive abilities are more widespread in literature. The possibility that cognitive impairment may develop as a consequence or aftermath of epilepsy was raised as early as 1885 when Gowers described 'epileptic dementia' as one of the pathological consequences of seizures. ${ }^{5}$ In the beginning of 
the $20^{\text {th }}$ century, various reports describe the so-called 'epileptic personality' which is supposedly characterized by being "emotional, suspicious, and irritable". ${ }^{6}$ Other adjectives that have been addressed to patients with epilepsy are "less intelligent, narrow-minded, inconsistent in thought and action, and of poorer memory". ${ }^{7}$ These often rather insulting characteristics were mostly based on subjective observations of those professionals working with patients with epilepsy. Fortunately, in that same era, researchers started to explore the more measurable aspects of cognitive deficits in patients with epilepsy. It became clear that patients with epilepsy can be grossly divided into three groups: those with normal cognitive abilities, those with initially normal cognition followed by cognitive decline and those with cognitive dysfunction at onset of epilepsy. In 1929, lower baseline intelligence rates and significant deterioration were found in the majority of children with epilepsy. ${ }^{6}$ A later study with adult patients presented less alarming data, with only moderate cognitive decline in about one-third of patients. ${ }^{8}$ Furthermore, it was observed that patients with socalled 'organic' or symptomatic epilepsy exhibited more frequently cognitive deterioration than those with idiopathic epilepsy. ${ }^{9}$

The abovementioned studies are only examples of a great amount of studies on cognition in epilepsy. Yet, some of the observations of more than a century ago are still valid today, and of extreme importance to our patients in daily practice.

\section{Cognitive co-morbidity}

Traditionally, research investigating cognitive co-morbidity in patients with epilepsy has focused on patients with temporal lobe epilepsy. In 1953, the now world-famous patient H.M. was treated for his intractable epileptic seizures with a resection of both medial temporal lobes. ${ }^{10}$ Thereafter, he suffered from severe anterograde amnesia, i.e., he could not store new information into his long-term memory system. Since then, the medial temporal lobe together with the hippocampus, are linked to memory deficits. Logically, when assessing cognitive problems in patients with temporal lobe epilepsy, the focus is on memory deficits, since both processes can be linked to the temporal lobes. However, other cognitive domains can also be disturbed in temporal lobe epilepsy, and epilepsy in general. These other cognitive domains include language, attention, and processing speed among others.

In clinical practice, cognitive problems can only be detected in such an advanced stage that memory deficits or reduced mental processing speed already interfere with normal functioning at school or at work. With conventional imaging techniques no changes in cerebral structures can be identified to explain the development or progression of cognitive deficits. With newer MRI techniques, such as functional MRI, a window to the neuronal basis of these problems is offered. This may provide insights into the possibility of early detection and screening of patients at risk for developing 
cognitive problems; as well as a potential for early intervention to slow down, stop or even prevent cognitive dysfunction.

\section{Functional magnetic resonance imaging}

Functional magnetic resonance imaging (fMRI) is based on the measurement of hemodynamic responses to certain stimuli, such as specific cognitive tests. The different magnetic properties of oxyhemoglobin and deoxyhemoglobin provide the basis for the increased signal seen in activated brain regions. This is referred to as the blood-oxygen-level-dependent (BOLD) contrast, ${ }^{11}$ and believed to reflect indirectly the underlying neuronal activity. ${ }^{12} \mathrm{fMRI}$ offers the possibility to localize cognitive processes quite accurately. On the other hand, a network of regions is often activated, whereas only a subset of regions is necessary for the task used. ${ }^{13}$ 


\section{Aim and outline of this thesis}

The aim of this thesis was to explore the possibilities to study cognitive deficits in patients with chronic epilepsy with fMRI. During the study period, fMRI analyzing techniques have evolved from the focus on functional activation patterns to the role of functional networks. Analyzing techniques range from predefined networks - such as the language network - to evaluation of the organization of the entire brain and measures for its efficiency. As mentioned previously, epilepsy is a heterogeneous disorder, and a wide variety of clinical factors can be identified to be involved in the development of cognitive deficits. The main research experiment was performed with patients with cryptogenic epilepsy, that is, in whom no symptomatic lesion had been visualized with regular imaging techniques. This way, the influence of a visible symptomatic lesion on the process of developing cognitive impairment could be ruled out.

In chapter 2, the rationale to apply $\mathrm{fMRI}$ in the investigation of cognitive deficits in chronic epilepsy, and results of previous fMRI investigations addressing cognitive functioning in epilepsy are reviewed.

A pilot study investigating patients with generalized seizures of different etiologies was performed. In chapter 3 , the possibility that cognitive deficits and $\mathrm{fMRI}$ activation patterns can be linked to the severity of the seizure disorder, expressed by the number of experienced seizures, is investigated.

The results of the main research experiment with a well-selected population of patients with cryptogenic localization-related epilepsy are described in chapters 4 through 7. Chapter 4 describes the technical background of quantitative examination of hippocampal and brain volumes in order to investigate whether reduced hippocampal volumes are implicated in memory deficits in patients without visible lesions in the temporal lobes. In chapter 5 , memory functions in our study population are assessed in relation with hippocampal and brain volumes, as well as with fMRIderived functional connectivity in predefined memory networks. The role of the language networks as measured with functional connectivity and its implications in language function is described in chapter 6 . The topological organization of the entire cerebral network is studied in chapter 7 , as well as the possibility that less efficiently organized networks in patients with epilepsy are associated with measures for cognitive decline.

Chapter 8 provides a general discussion of this thesis and future implications of the current results. 


\section{References}

1. Blume WT, Luders HO, Mizrahi E, Tassinari C, van Emde Boas W, Engel J, Jr. Glossary of descriptive terminology for ictal semiology: Report of the ILAE task force on classification and terminology. Epilepsia. 2001;42:1212-8.

2. Panayiotopoulos CP. A clinical guide to epileptic syndromes and their treatment. 1st ed. Oxford: Bladon Medical Publishing; 2002.

3. Nationaal Epilepsie Fonds. www.epilepsie.nl. 2009

4. Commission on Classification and Terminology of the International League Against Epilepsy. Proposal for revised classification of epilepsies and epileptic syndromes. Epilepsia. 1989;30:389-99.

5. Gowers WR. Epilepsy and other chronic convulsive diseases: Their causes, symptoms and treatment. New York: William Wood \& Co; 1885.

6. Dawson S, Conn JCM. The intelligence of epileptic children. Arch Dis Child. 1929; 4:142-50.

7. Wiersma ED. The psychology of epilepsy. Journal of Mental Science. 1923;69:482-97.

8. Fetterman J, Barnes MR. Serial studies of the intelligence of patients with epilepsy. Arch Neurol Psych. 1933:797-800.

9. Arieff AJ, Yacorzynski GK. Deterioration of patients with organic epilepsy. J Nerv Ment Dis. 1942;96: 49-55.

10. Scoville WB, Milner B. Loss of recent memory after bilateral hippocampal lesions. J Neurol Neurosurg Psychiatry. 1957;20:11-21.

11. Ogawa S, Lee TM, Kay AR, Tank DW. Brain magnetic resonance imaging with contrast dependent on blood oxygenation. Proc Natl Acad Sci U S A. 1990;87:9868-72.

12. Logothetis NK, Pauls J, Augath M, Trinath T, Oeltermann A. Neurophysiological investigation of the basis of the fMRI signal. Nature. 2001;412:150-7.

13. Powell HW, Koepp MJ, Richardson MP, Symms MR, Thompson PJ, Duncan JS. The application of functional MRI of memory in temporal lobe epilepsy: A clinical review. Epilepsia. 2004;45:855-63. 



\section{Chapter}

Functional MRI in chronic epilepsy: associations with cognitive impairment

MCG Vlooswijk, JFA Jansen, MCFTM de Krom, HJM Majoie, PAM Hofman, WH Backes, AP Aldenkamp Lancet Neurology 2010;9:1018-1027 


\section{Summary}

Chronic epilepsy is frequently accompanied by serious cognitive side-effects. Clinical factors are important, but cannot account entirely for this cognitive comorbidity. Therefore, research is focusing on the underlying cerebral mechanisms to understand the development of cognitive dysfunction. In the past two decades, functional MRI techniques have been applied extensively to the study of cognitive impairment in chronic epilepsy. However, because of wide variation in study designs, analysis methods, and data presentation, interpretation of these studies has become increasingly difficult for clinicians. In patients with localizationrelated epilepsy, whether findings of functional MRI represent the underlying neuronal substrate for cognitive decline remains a subject of debate. 


\section{Introduction}

Although seizures are the most prominent feature of epilepsy, many patients rank cognitive impairment highest on their list of complaints (Table 2.1). ${ }^{1-3}$ The cognitive disorders reported in epilepsy cover essentially all cognitive domains. In temporal lobe epilepsy (TLE), memory deficits of all kinds have been described, especially impairment of verbal long-term consolidation and retrieval, verbal learning, shortterm or working memory, ${ }^{4}$ and spatial memory. ${ }^{5}$ However, other neocortical functions can also be affected in focal epilepsy, causing frontal lobe dysfunctions, such as problems with response inhibition, attention, planning, and psychomotor speed. ${ }^{5}$ Furthermore, language deficits, such as poor naming and fluency skills and disturbance of global intelligence, can occur. ${ }^{6-8}$ Most of this information is from studies in patients with TLE $^{9}$ because this disorder is the most frequent cause of medically refractory epilepsy, and epilepsy surgery is often considered in these patients. In preoperative screening, a large amount of data is collected, with extensive information about seizure focus, language dominance, and neuropsychological profile. $^{10}$

Table 2.1 Major disorders in patients with epilepsy.

\begin{tabular}{|c|c|}
\hline & $\begin{array}{l}\text { Patients reporting moderate-to- } \\
\text { severe disorders }\end{array}$ \\
\hline \multicolumn{2}{|l|}{ Mental health } \\
\hline Fear of recurring seizures & $32-73 \%^{*+}$ \\
\hline Change of outlook for future plans and ambitions & $28-47 \%^{\ddagger \ddagger}$ \\
\hline (Fear of) stigma & $40-51 \%^{\dagger \ddagger}$ \\
\hline \multicolumn{2}{|l|}{ General health } \\
\hline Lack of energy & $22-47 \%^{\ddagger \ddagger}$ \\
\hline Side-effects or adverse effects from an antiepileptic drug & $88 \%^{\ddagger}$ \\
\hline \multicolumn{2}{|l|}{ Cognitive functions } \\
\hline Impaired memory; as an adverse effect of an antiepileptic drug & $46 \%^{*} ; 50 \%^{\ddagger}$ \\
\hline Impaired concentration; as an adverse effect of an antiepileptic drug & $46 \%^{*} ; 48 \%^{\ddagger}$ \\
\hline Impaired thinking; as an adverse effect of an antiepileptic drug & $40 \%^{*} ; 40 \%^{\ddagger}$ \\
\hline \multicolumn{2}{|l|}{ Lifestyle } \\
\hline Impaired ability to drive a car & $36 \%{ }^{*}$ \\
\hline Adverse effect on leisure pursuit & $31 \%^{+}$ \\
\hline \multicolumn{2}{|l|}{ Relationships } \\
\hline Adverse effect on relations with family and close others & $28-41 \%{ }^{* \dagger \ddagger}$ \\
\hline Negative effect on sex life & $21 \%{ }^{*}$ \\
\hline Concerns about having children & $39 \%^{*}$ \\
\hline \multicolumn{2}{|l|}{ Social activities } \\
\hline Adverse effect on school performance & $58 \%{ }^{*}$ \\
\hline Adverse effect on job performance & $17-38 \%{ }^{*+\ddagger}$ \\
\hline
\end{tabular}


Naturally, much research has focused on the factors contributing to the development of cognitive dysfunction. Ultimately, if a clinical factor could be identified to play a major part in this development, clinicians might stop or change this process by tailoring the treatment of their patients. In this respect, research has focused on the role of seizure frequency, seizure severity, ${ }^{11}$ chronic antiepileptic drug use, ${ }^{12}$ and persistent interictal epileptic brain activity. ${ }^{13}$ Other factors that could affect cognition in epilepsy are seizure-induced head trauma, ${ }^{14}$ young age at onset of epilepsy, ${ }^{15}$ and long duration of epilepsy. ${ }^{8}$ However, since cognitive deficits can be already present in newly diagnosed, untreated patients, ${ }^{16,17}$ clinical factors, such as high frequency or severity of seizures, are unlikely to account completely for the reported cognitive disorders. The focus of research has therefore changed to the neuronal mechanisms responsible for cognitive deficits.

Since the introduction of MRI, several techniques have been applied in research focusing on the cerebral processes responsible for cognitive decline. Functional MRI (fMRI) has been used extensively to investigate cognitive deficits because it can combine data from cognitive $\mathrm{fMRI}$ studies with neuropsychological data to display the dynamics of cognitive processes. In the past two decades, many fMRI studies have been published. However, most studies focus on a fairly specific area of cognitive functioning or the brain. In this Review, we summarize and put into perspective research on $\mathrm{fMRI}$ abnormalities associated with cognitive deficits in localizationrelated epilepsy, and discuss the extent to which $f M R I$ findings represent the underlying neuronal substrate that might explain cognitive decline. Furthermore, we will discuss the limitations of $\mathrm{fMRI}$ techniques and make recommendations for future research.

\section{fMRI activation patterns}

fMRI has been used extensively in preoperative screening to predict possible postoperative cognitive deficits, so many patients with localization-related epilepsy have undergone both $\mathrm{FMRI}$ and neuropsychological assessment. ${ }^{18}$ These data can be analyzed to investigate the extent to which fMRI patterns correlate with cognitive tests. In this chapter, we will focus on fMRI studies that specifically mention the correlation between neuropsychological data and fMRI activation patterns (Table 2.2). ${ }^{19-33}$

Theoretically, four possible activation patterns can be recorded in investigation of cognitive deficits in patients with epilepsy. First, activation patterns could be similar for patients and healthy controls despite cognitive differences; these types of results are not likely to be published. 


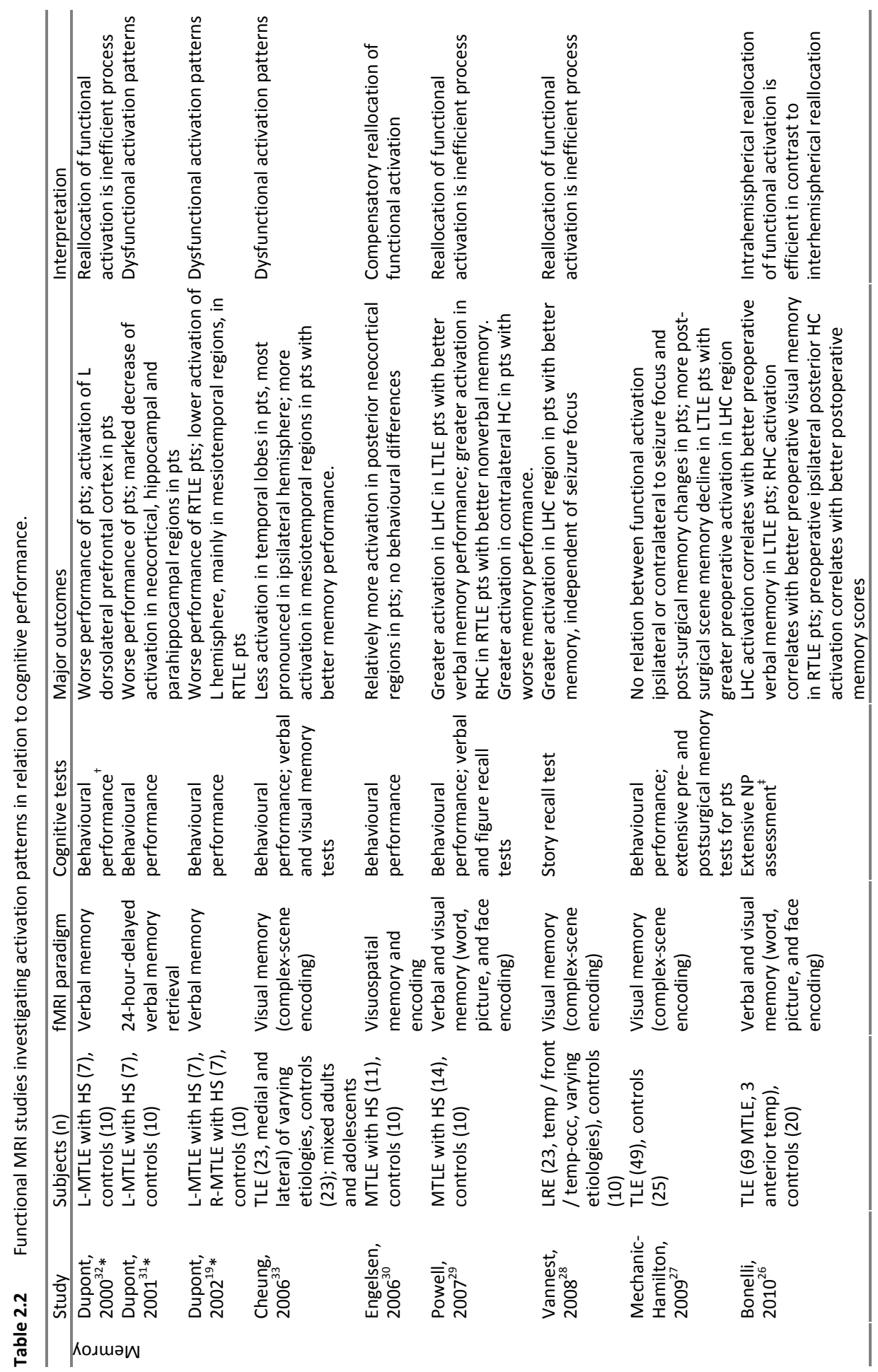




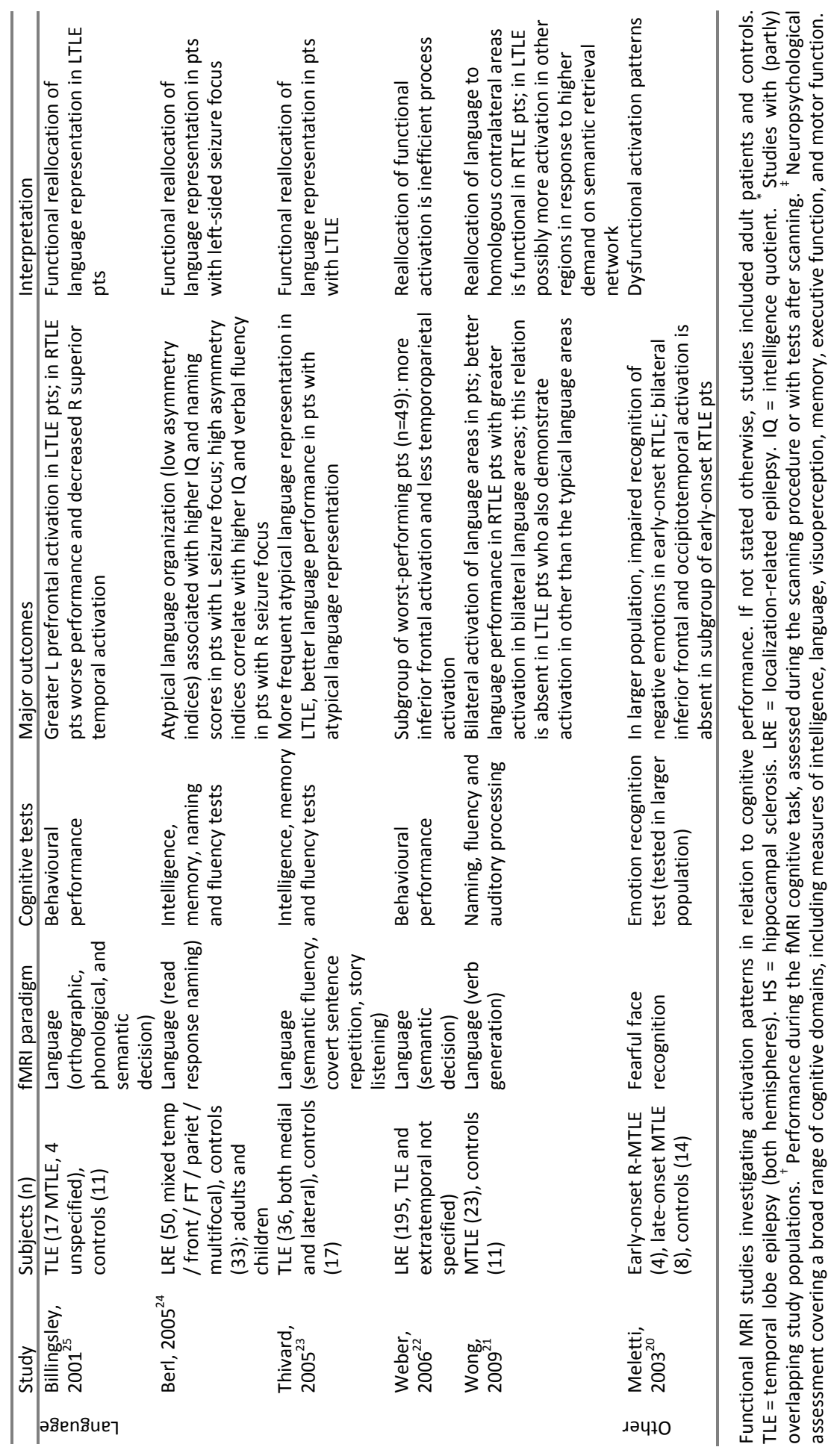


Second, an absence or decrease in activation could be recorded in areas that are activated in healthy controls. Third, more activation could be recorded in patients than in healthy controls, either in or outside the brain regions activated in controls. Fourth, the second and third patterns could be combined, resulting in less activation in one area and more activation in another, suggesting a shift of activation. The second and fourth patterns of activation predominate in studies in which fMRI abnormalities are described in relation to cognitive data. Dependent on whether cognitive deficits are present or not, the changes in activation patterns can be interpreted as either a functional or dysfunctional process. A functional process represents an effective, possibly compensatory mechanism to preserve cognitive functioning. A dysfunctional process represents a pathological or at least an insufficient compensatory mechanism.

\section{Memory}

Most studies included in this chapter were done in patients with unilateral TLE, and many of these used verbal memory tasks, ${ }^{19,31,32,34}$ visual complex-scene encoding tasks, ${ }^{27,28,30,33}$ or a combination of both. ${ }^{26,29}$ In one study, patients with left-sided TLE showed activation of the left dorsolateral prefrontal cortex in addition to the bilateral activation of the parahippocampal gyri seen in healthy controls. ${ }^{32}$ In subsequent studies with overlapping samples, patients were rescanned 24 hours later during a verbal memory retrieval task, and, unlike controls, they did not have increased activation of the right hippocampus or parietal brain areas. ${ }^{31}$ Interestingly, patients with right-sided TLE also performed more poorly on the verbal memory task than did controls, and this was associated with lower functional activation in the left mesiotemporal lobe. ${ }^{19}$ Although the sample sizes in these studies were small, these data suggest that functional activation is reallocated in patients with left TLE, and patients with right TLE have a bilateral functional impairment. However, the data should be interpreted with care, since fMRI data were only correlated with performance during the $\mathrm{FMRI}$ task, and not with results of formal neuropsychological tests. Furthermore, patients with left TLE were included in these studies only when verbal memory dysfunction was present, and $\mathrm{fMRI}$ activation patterns could have been affected by the difference in task performance between patients and controls.

Similar results were reported in a somewhat larger population of patients with left and right TLE, in which better memory was correlated with greater activation in the mesial temporal lobes. ${ }^{19}$ However, no left-right differentiation was noted for verbal and visual memory. In a subsequent study, less activation in the damaged left hippocampus was correlated with worse verbal memory in a group of patients with left TLE. ${ }^{29}$ A similar effect was recorded for non-verbal memory in patients with right TLE: the greater the activation of the right hippocampus, the better the non-verbal memory performance. ${ }^{29}$ Even though this study was small, the findings suggest that reorganization of memory functions to the undamaged mesiotemporal lobe is an 
inefficient process. This explanation would account for the fact that patients with better preoperative memory functioning, and probably a better functioning hippocampus on the side of the seizure focus, are most at risk of postoperative impairment when the epileptogenic hippocampus is removed. In a large surgical population, better preoperative verbal memory was correlated with greater left hippocampal activation for word encoding in patients with left TLE (Figure 2.1). ${ }^{26}$ In patients with right TLE, greater right hippocampal activation during face encoding was correlated with better visual memory. Furthermore, higher preoperative activation of the posterior part of the ipsilateral hippocampus in left and right TLE was correlated with better verbal and visual memory scores, respectively, after anterior temporal lobe resection (in which only the anterior part of the hippocampus is resected).
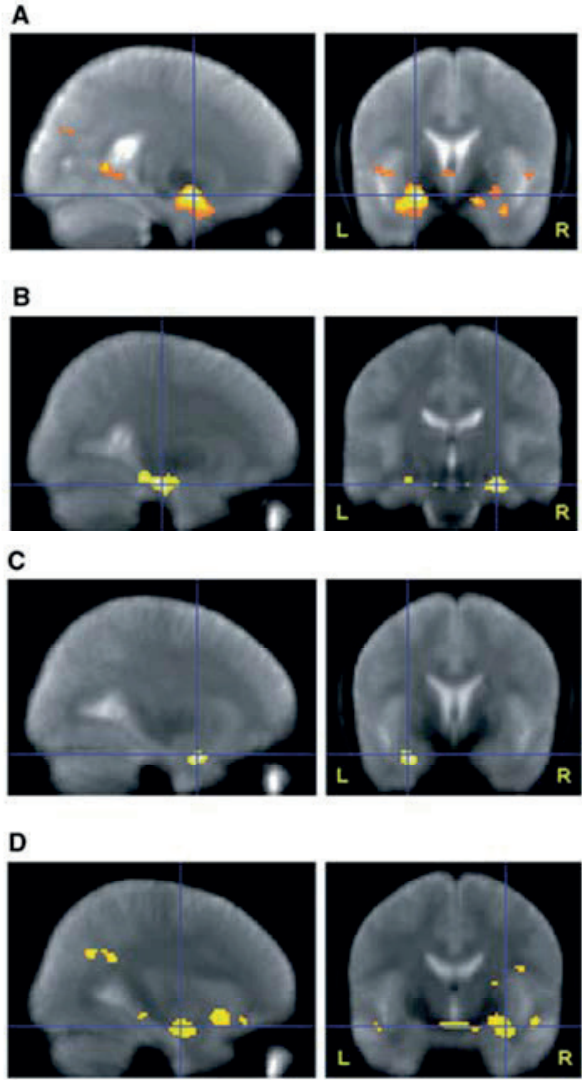

Figure 2.1 Example of functional MRI activation patterns for word encoding (A, B) and face encoding (C, D) in healthy controls and patients with left-sided or right-sided temporal lobe epilepsy.

The left side of the brain is shown on the left side of the coronal images. The main effects in controls are left hippocampal activation for word encoding (A) and right hippocampal activation for face encoding (C). Patients with left TLE show less left hippocampal activation for word encoding (B), and patients with right TLE show less right hippocampal activation for face encoding (D) than do controls. TLE = temporal lobe epilepsy. Reproduced from Bonelli and colleagues, ${ }^{27}$ with permission from Oxford University Press. 
These results support the so-called functional adequacy model, which suggests that postoperative memory function is better preserved with recruitment of ipsilateral posterior hippocampal networks than with recruitment of the contralateral hippocampus. Indirect support for this theory comes from another surgical study, in which higher preoperative activation in the left hippocampal region in patients with left TLE correlated with greater postoperative visual memory decline; ${ }^{27}$ a direct comparison between $\mathrm{fMRI}$ activation and preoperative memory performance was not done. In a mixed population of patients with localization-related epilepsies, patients with a left-lateralized seizure focus showed a shift towards right-lateralized activation. $^{28}$ To a lesser extent, a similar effect was seen for patients with a righthemisphere seizure focus, who had more left-lateralized activation. However, for both patient groups, better verbal memory was associated with greater left-lateralized mesial temporal lobe activation. Hence, the shift of activation from the left to the right hemisphere was not sufficient to maintain normal verbal memory. ${ }^{28}$

Recognition of faces with negative emotional expressions is less frequently studied than are other types of non-verbal memory. In a subgroup of patients with right-sided TLE of early onset, impaired recognition of facial expressions correlated with the absence of activation in the inferior frontal cortex and occipitotemporal cortex bilaterally. $^{20}$

By contrast with most studies describing dysfunctional changes in activation patterns-ie, not sufficient to maintain normal memory-some studies support the theory that functional activation changes are an efficient compensatory mechanism. For instance, Engelsen and colleagues ${ }^{30}$ did not record any behavioural differences between patients and controls during a visuospatial encoding and retrieval test. They did, however, show altered fMRI activation patterns, with more occipitoparietal activation in patients and more prefrontal and parietal activation in healthy controls. The investigators postulated that patients showed compensatory activation in the occipitoparietal parts of the brain, but, again, no extensive neuropsychological assessment was done to test whether memory was indeed undisturbed in the patient group. A similar study design was applied in a small population in which both patients and controls had intact verbal memory, ${ }^{34}$ and patients showed more activation in some regions, which was interpreted as a compensatory mechanism to maintain normal verbal memory.

\section{Language processing}

Language representation and its possible reorganization are of major importance in preoperative screening. In several studies, activation patterns in response to language tasks have been correlated with language performance. During two linguistic fMRI tasks, functional activation patterns substantially overlapped in patients with 
unilateral TLE. However, in patients with left-sided TLE, the left dorsolateral prefrontal cortex had greater activation than in healthy controls, as well as increased signal change in the left inferior frontal and right middle temporal gyri. ${ }^{25}$ Surprisingly, only the patients with right TLE had poorer performance on the linguistic tasks than did controls, as well as decreases in right superior temporal activation. The investigators argued that the higher left frontal activity in left TLE suggested a functional reorganization of language representation. However, the linguistic tasks might not have been sensitive enough to detect clinically relevant language deficits, and therefore the results should be interpreted with care.

In a heterogeneous preoperative population, patients with worse performance during a linguistic fMRI task-i.e., those with lower accuracy and longer reaction timesshowed greater activation in the inferior frontal region and less activation in the temporoparietal region than did patients who performed better on this task. ${ }^{22}$ Language functions, such as verbal fluency and naming scores, correlated with degree of language reorganization and side of seizure focus in a large, heterogeneous group of patients: ${ }^{24}$ good preservation of language functions was associated with normal language representation, expressed as high asymmetry indices, in patients with a right-sided seizure focus, but with atypical language representation and low asymmetry indices in those with a left-sided focus. Similarly, in a small population of patients with TLE, atypical language representation was more frequently recorded in left TLE than in right TLE. ${ }^{23}$ Patients with atypical language representation performed better on language tests than did those with normal language representation, which is indicative of an adaptive, compensatory mechanism. In Wong and colleagues' study, ${ }^{21}$ greater bilateral activation in the typical language regions was correlated with better language in patients with right TLE. The researchers suggested that a similar effect was not recorded in the left TLE group because language processing had been reallocated to regions other than those investigated.

\section{Clinical and theoretical implications and limitations}

Differences in study populations, fMRI cognitive tasks, and outcome measures make identification of a general effect of localization-related epilepsy on fMRI activation maps and cognition extremely difficult. Some studies have included patients partly on the basis of the presence of cognitive deficits, ${ }^{32}$ but altered functional activation is difficult to correlate directly with these deficits. In others, poorer neuropsychological performance and altered functional activation are reported, but direct correlations were not included. ${ }^{27}$ In several studies, the fMRI activation patterns have been correlated with the behavioural data obtained during fMRI scanning, but these data might not be as sensitive as the results of formal neuropsychological testing for detection of cognitive deficits. Furthermore, most of these studies investigated small populations with epilepsy. 
Nevertheless, most studies support one of two possible processes in patients with localization-related epilepsy: activation is decreased in the regions that are activated in healthy controls and is associated with cognitive deficits, or activation is intrahemispherically or interhemispherically shifted from the normally activated regions to other cerebral regions. Data are contradictory as to whether this shift is functional (with preserved cognitive abilities) or dysfunctional (with cognitive impairment). Most studies support a dysfunctional shift: for example, in a small heterogeneous population with epilepsy, a shift from frontotemporal to prefrontal activation had some correlation with cognitive decline. ${ }^{35}$ Conversely, associations between reported functional activation patterns and cognitive deficits do not necessarily signify a causal relationship between both factors-differences in fMRI activation patterns and cognitive abnormalities could be the result of an unknown third, etiological factor.

In daily practice, fMRI activation maps can be applied in preoperative screening to identify patients with high risk of postoperative cognitive deficits. From the studies reviewed, we conclude that patients with the most interhemispheric or intrahemispheric reorganization of cognitive processes are least likely to have poor postoperative cognitive outcome. However, these patients are more likely to have poorer preoperative cognitive function. Present $\mathrm{fMRI}$ techniques are not yet sensitive enough to be used as a diagnostic or prognostic instrument in individuals with cognitive disorders.

\section{Functional connectivity}

\section{Analysis methods}

In the past ten years, research has started to focus on the function of cerebral networks in epilepsy. Cognitive functions depend on the orchestrated action of several rather than isolated brain regions. For that reason, exploration of the integrity and organization of cerebral networks could reveal more about cognitive decline than could study of activation patterns. For TLE, abnormal function seems to extend from the epileptic zone in the temporal lobe to connected regions within and outside the temporal lobe. ${ }^{36}$ Increasing interest is being given to the effects of dysfunctional networks on cognition in epilepsy.

With $\mathrm{fMRI}$, different analysis techniques have been applied to measure functional connectivity. First, the extent to which the time course of the fMRI signal is correlated between different cerebral regions can be measured. ${ }^{37}$ High correlation coefficients are indicative of high functional connectivity between the selected regions. This technique has been used with resting-state fMRI to investigate the language- 
processing network, and showed that patients with left TLE had a disruption to their language network compared with controls. ${ }^{38}$ Another study used a visual memory task to investigate the default mode network (i.e., a group of brain regions that are more active in passive than in active tasks) and the role of the precuneus (part of the superior parietal lobule). ${ }^{39}$ Patients with left TLE had weaker functional connectivity between the precuneus and ipsilesional lateral temporal cortex and hippocampus, which the researchers interpreted as damage to the network distant from the site of seizure focus.

A second method to investigate functional connectivity within a network is independent component analysis. The brain is divided into different networks (components) of brain regions on the basis of the extent to which the signal time courses of these regions coincide, ${ }^{40}$ and the contribution of individual brain regions to this network can then be calculated. This technique was used in patients with unilateral TLE to select the component that best fitted the template of the default network. ${ }^{41}$ Patients with left-sided TLE had weaker functional connectivity in the left mesial temporal structures, whereas patients with right-sided TLE had lower connectivity in both mesial temporal lobes. In a study of the networks for auditory, sensorimotor, and visual information processing in patients with bilateral TLE, patients had lower auditory and sensorimotor functional connectivity than did controls. ${ }^{42}$ By contrast, within the visual network, patients had stronger functional connectivity in the primary visual cortex and a corresponding decrease in higher order visual cortex.

A third technique to investigate functional connectivity does not depend on previous selection of the network of interest. The brain is divided into several regions-eg, 90 regions with anatomical automatic labelling ${ }^{43}$-and graph theoretical analysis is used to investigate the topology of the network (for a review, see Stam and Reijneveld ${ }^{44}$ ) and estimate whether the brain exhibits small-world characteristics or represents a random network. Small-world characteristics refer to widespread local clustering, combined with some long-distance connections for rapid information transfer throughout the overall network. Random networks are supposed to be less efficient in global communication than are small-world characteristics, and have been associated with lower intelligence in healthy adults. ${ }^{45}$ With this technique, patients with bilateral TLE were shown to have more randomly structured networks than were healthy controls. ${ }^{46}$ Notably, combination of studies investigating small-world characteristics could be used to explore the possible relation between cognitive dysfunction and altered organization of cerebral networks in patients with chronic epilepsy.

\section{Combination of functional connectivity and cognitive assessment}

According to the abovementioned studies, network functionality is decreased in TLE, but the effect of these network changes on cognitive functioning has not been 
investigated. In most studies addressing both functional connectivity and cognitive functions, a predefined network of interest was used and the correlation coefficients of signal time courses within this network were compared with the results from cognitive tests.

In a surgical population of patients with TLE, higher preoperative functional connectivity between the hippocampus and the superior temporal gyrus was noted for patients who had decreased verbal learning postoperatively than for those who remained stable or improved postoperatively. ${ }^{47}$ The investigators suggested that greater functional connectivity is indicative of higher functional network integrity. In a small sample of patients with left TLE, functional connectivity within the temporal lobes was investigated. Compared with controls, patients had less functional connectivity within the left mesiotemporal structures. ${ }^{48}$ Furthermore, patients with greater functional connectivity within the right hippocampus had better memory than did those with less functional connectivity, which was interpreted as a compensatory mechanism. $^{48}$ By contrast, in patients with unilateral TLE, higher functional connectivity between both hippocampi was not associated with better test performance than in those with low functional connectivity between the hippocampi. $^{49}$

In the autobiographical network, the connections with the left hippocampus were weaker in patients with left TLE than in controls. ${ }^{50}$ Conversely, functional connectivity between regions outside the hippocampus was higher in these patients than in controls. This bypassing of the damaged hippocampal node was interpreted as a compensatory mechanism, especially because patients had only a mild deficit of episodic autobiographical memory. In the study described in chapter $6^{37}$ of patients with cryptogenic localization-related epilepsies, impairment of language function was associated with decreased functional connectivity within the prefrontal network. With independent component analysis, lower functional connectivity in the dorsal attention network was associated with worse attention in patients with bilateral TLE. ${ }^{51}$ Moreover, better attention was correlated with stronger connectivity in the right frontal eye field, indicating a compensatory mechanism.

\section{Structural and functional connectivity}

Although $\mathrm{fMRI}$ can be used to measure whether two brain regions are functionally connected by correlation of the signal time courses of these regions, this functional connection does not necessarily imply a structural connection. ${ }^{44}$ To investigate structural connectivity, diffusion-tensor imaging can be used. With this MRI technique, measurement of the diffusion of water molecules in brain tissue can be used to visualize cerebral white matter tracts (magnetic resonance tractography). In patients with TLE, functional language lateralization correlates with structural 
lateralization. ${ }^{52}$ Both functional and structural connections were greater in the right hemisphere in patients with left TLE than in patients with right TLE and controls, but the reverse was seen in patients with right TLE (Figure 2.2). ${ }^{52} \mathrm{~A}$ similar structuralfunctional coupling (i.e., left lateralization) was seen in the arcuate fasciculus - a white matter tract connecting the anterior and posterior language regions-of patients with right TLE, but not in patients with left TLE. ${ }^{53}$

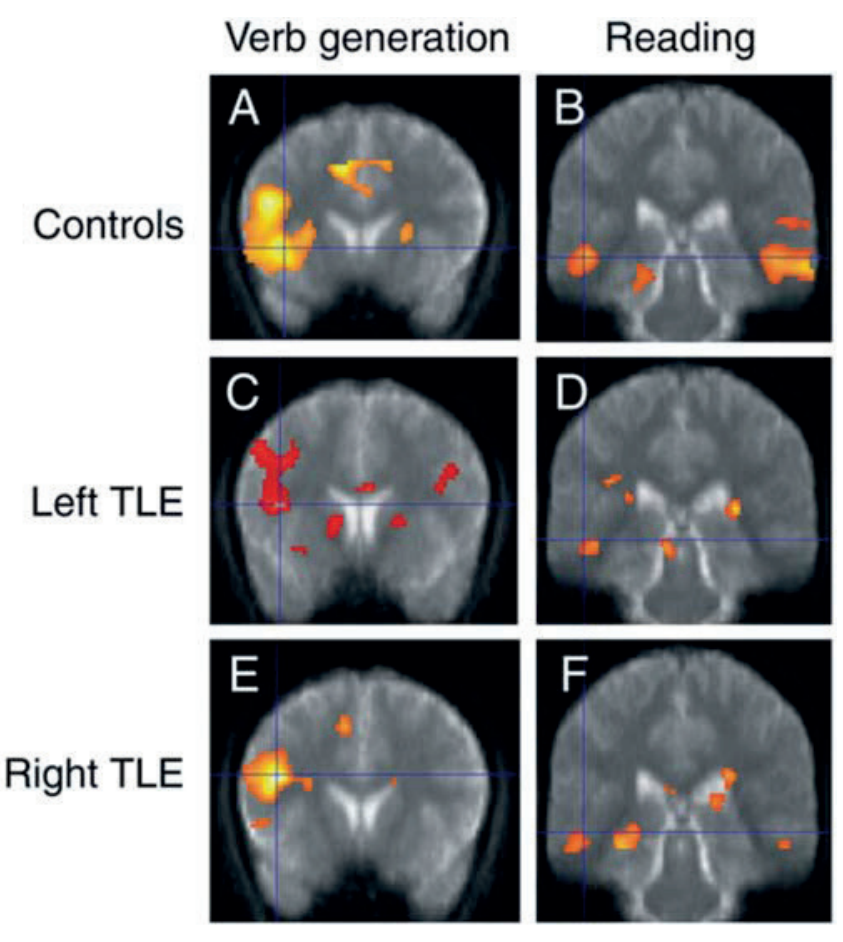

Figure 2.2 Example of functional $M R I$ activation patterns for verb generation and reading comprehension in controls (A, B) and patients with left-sided (C, D) or right-sided ( $E, F)$ temporal lobe epilepsy.

Significant regions are superimposed onto the normalized mean structural MRI scans from all controls or patients. The left side of the brain is shown on the left side of the images. For verb generation, controls (A) and patients with right TLE (E) show activation of the left inferior frontal gyrus, whereas patients with left TLE (C) show bilateral activation of the inferior frontal gyrus. For reading comprehension, controls (B) and patients with right TLE (F) show bilateral activation of the superior temporal gyrus, whereas patients with left TLE (D) show activation of the left superior temporal gyrus. TLE = temporal lobe epilepsy. Reproduced from Powell and colleagues, ${ }^{52}$ with permission from Elsevier.

Some studies have combined results of diffusion-tensor imaging with neuropsychological data. In patients with left TLE, the integrity of the uncinate fasciculus-a major white matter tract connecting anterior temporal and frontal 
lobes-was related to memory performance. ${ }^{54}$ In a small TLE sample, higher lateralization of structural connections from the inferior frontal gyrus was correlated with better language performance. ${ }^{55}$ For patients with TLE and interictal psychosis, poorer neuropsychological performance was related to disturbed frontotemporal white matter integrity, as indicated by fractional anisotropy measures derived from diffusion-tensor imaging. ${ }^{56}$ In a study comparing patients with both left and right TLE versus controls, poor verbal memory and naming were correlated with structural damage to many fiber tracts, especially in the language-dominant hemisphere. ${ }^{57}$ This effect was specific to selected white matter tracts because cognitive performance was not related to the integrity of a motor white matter tract. However, the sample size was small and heterogeneous, with inclusion of some patients after anterior temporal lobe resection.

\section{Other techniques to study functional connectivity}

fMRI is useful for study of functional connectivity because of its high spatial resolution. However, temporal resolution is low by contrast with other techniques, such as magnetoencephalography and electroencephalography (EEG). With these techniques, functional connectivity can be measured by correlation of time series of regions of neuronal activity in terms of wavelet frequencies. ${ }^{44}$ In epilepsy, EEG connectivity analyses have been used to study epileptogenic networks, ${ }^{58-60}$ indicating a localized increase in synchronization during seizures and decreased functional connectivity in the interictal state. Recent technological advances have allowed the simultaneous acquisition of EEG and $\mathrm{FMRI}$, which yields a powerful strategy combining the high spatial resolution of $\mathrm{FMRI}$ with the high temporal resolution of EEG. ${ }^{61}$ The interesting theory that these changes in synchronization and functional connectivity might participate in the development of interictal cognitive deficits in epilepsy has, however, not been tested.

\section{Clinical and theoretical implications and limitations}

Functional connectivity analysis is a fairly new, but promising, method to study cognitive dysfunction in epilepsy. Studies with this technique all point to a disruption of functional networks in epilepsy, either in a small region or in the whole brain. However, this conclusion is based on mean results for patient groups, which limits application to individual patients with cognitive disorders.

Whether functional connectivity studies are better done with task-related or restingstate $\mathrm{fMRI}$ is not yet certain. With resting-state $\mathrm{fMRI}$, measurement of spontaneous fluctuations in the blood oxygen level dependent (BOLD) signal provides an indication of spontaneous neuronal activity. Accumulating evidence suggests that spontaneous BOLD fluctuations map specifically to functionally related brain regions and relate to known anatomical networks. ${ }^{62}$ Since resting-state $\mathrm{fMRI}$ does not depend on task 
performance, it can be applied more extensively than can task-related fMRI. However, resting itself is also associated with a certain amount of attention, hence a certain cognitive state, which is indicated by activation of the typical default network. ${ }^{62}$

Different analysis methods have been used to investigate functional connectivity. Most studies have used analysis methods to explore functional connectivity within a predefined network, including regions known to participate in a specific cognitive function. Although this approach is rational for exploration of potentially disrupted networks, it cannot show network reorganization associated with brain regions excluded from the analysis. Furthermore, most studies describe disrupted functional connectivity in terms of decreased statistical interdependencies between signal time courses of different brain areas. However, analysis of $\mathrm{fMRI}$ data to construct a hierarchical map and identify which regions are most closely related is more complicated. ${ }^{62}$ Attempts to explore hierarchical relations within a network also depend on an a-priori definition of regions of interest. On the contrary, with independent component analysis, predefinition of the network of interest is not needed. However, the researcher needs to decide which one of the networks identified by mathematical algorithms is a neuroanatomical system, thereby introducing a selection bias. Also, disentangling of all physiological (respiratory, cardiac, and vasomotion) and motion confounds through preprocessing is very challenging, and remains a major difficulty. ${ }^{63}$ Graph theoretical analysis offers the opportunity to study the entire brain network in terms of effective network topology. This fairly new technique is developing quickly and its applicability in epilepsy and cognition needs further exploration in the near future.

\section{Conclusions and future directions}

Cognitive dysfunction in localization-related epilepsies is associated with changes in functional activation patterns and decreased functional connectivity within selected brain networks. fMRI is especially suited to research of cognitive consequences of epilepsy because it can display the dynamics of cognitive processes. However, direct comparison between most studies is complicated because of differences in study populations, fMRI tasks, and methods for data analysis. Research is now focusing on the effect of epileptic processes on localized and global cerebral networks.

On the basis of existing knowledge of cerebral processes underlying cognitive dysfunction in epilepsy, future studies are needed to address some unanswered questions. $\mathrm{fMRI}$ could be used to investigate chronic extratemporal epilepsies in large populations, and thereby establish whether knowledge about patients with TLE can be extended to patients with other epilepsy types or whether other cerebral processes 
are occurring. The role of altered cerebral networks in the development of cognitive deficits in epilepsy needs extensive exploration.

Ideally, longitudinal studies should be done to understand changes in network organization in relation to cognitive functioning, with retesting intervals of several years. The fact that cognitive impairment is already present at onset of epilepsy in some patients implies that unknown, pathological cerebral processes have occurred. However, we have not identified any information about the order in which cognitive dysfunction, and structural and functional MRI abnormalities, occur. Study of large healthy populations to capture patients who develop chronic epilepsy is unfeasible, but researchers should try to study patients from the onset of disease. Preferably, the effect of clinical factors, such as antiepileptic drug use, epileptiform discharges, and seizure frequency, on these networks and cognition should be assessed. The association between cognitive outcome and other factors, such as age at onset of epilepsy, underlying pathology, seizure control with antiepileptic drugs or surgery, age at seizure control, and duration of epilepsy, needs investigation.

Information about factors that affect seizure control and cognitive outcome could provide a window of opportunity for effective seizure control to avoid cognitive impairment. The cerebral reserve theory-i.e., higher intellect before morbidity can protect against cognitive decline ${ }^{64}$-raises the issue of whether neurocognitive training at an early stage might reinforce existing networks and thus slow down or prevent cognitive decline. Identification of the cerebral mechanisms underlying the development of cognitive disorders, and the patients at risk for cognitive deterioration, might eventually lead to earlier therapeutic intervention than is available at present, with better cognitive outcome.

\section{Search strategy and selection criteria}

We searched PubMed for articles published between Jan 1, 1929, and June 23, 2010, with the terms "epilepsy", "functional MRI", "cogniti*", "memory", and "language". We included articles identified from these searches and relevant references cited in the articles. Only articles published in English were included. We omitted studies in which fMRI results were presented without addressing correlations with neuropsychological data. 


\section{References}

1. Chaplin JE, Yepez Lasso R, Shorvon SD, Floyd M. National general practice study of epilepsy: the social and psychological effects of a recent diagnosis of epilepsy. BMJ. 1992;304:1416-8.

2. Fisher RS, Vickrey BG, Gibson P, Hermann B, Penovich P, Scherer A, Walker S. The impact of epilepsy from the patient's perspective I. Descriptions and subjective perceptions. Epilepsy Res. 2000;41: 39-51.

3. Baker GA, Jacoby A, Buck D, Stalgis C, Monnet D. Quality of life of people with epilepsy: a European study. Epilepsia. 1997;38:353-62.

4. Helmstaedter C. Effects of chronic epilepsy on declarative memory systems. Prog Brain Res. 2002;135:439-53.

5. Axmacher N, Schmitz DP, Wagner T, Elger CE, Fell J. Interactions between medial temporal lobe, prefrontal cortex, and inferior temporal regions during visual working memory: a combined intracranial EEG and functional magnetic resonance imaging study. J Neurosci. 2008;28:7304-12.

6. Hoppe C, Elger CE, Helmstaedter C. Long-term memory impairment in patients with focal epilepsy. Epilepsia. 2007;48 Suppl 9:26-9.

7. Oyegbile TO, Dow C, Jones J, Bell B, Rutecki P, Sheth R, Seidenberg M, Hermann BP. The nature and course of neuropsychological morbidity in chronic temporal lobe epilepsy. Neurology. 2004;62: 1736-42.

8. Jokeit H, Ebner A. Effects of chronic epilepsy on intellectual functions. Prog Brain Res. 2002;135: 455-63.

9. Elger CE, Helmstaedter C, Kurthen M. Chronic epilepsy and cognition. Lancet Neurol. 2004;3:663-72.

10. Baxendale S. The Wada test. Curr Opin Neurol. 2009;22:185-9.

11. Dodrill CB. Progressive cognitive decline in adolescents and adults with epilepsy. Prog Brain Res. 2002;135:399-407.

12. Vermeulen J, Aldenkamp AP. Cognitive side-effects of chronic antiepileptic drug treatment: a review of 25 years of research. Epilepsy Res. 1995;22:65-95.

13. Aldenkamp A, Arends J. The relative influence of epileptic EEG discharges, short nonconvulsive seizures, and type of epilepsy on cognitive function. Epilepsia. 2004;45:54-63.

14. Thompson PJ, Duncan JS. Cognitive decline in severe intractable epilepsy. Epilepsia. 2005;46:1780-7.

15. Hermann B, Seidenberg M, Bell B, Rutecki P, Sheth R, Ruggles K, Wendt G, O'Leary D, Magnotta V. The neurodevelopmental impact of childhood-onset temporal lobe epilepsy on brain structure and function. Epilepsia. 2002;43:1062-71.

16. Taylor J, Kolamunnage-Dona R, Marson AG, Smith PE, Aldenkamp AP, Baker GA. Patients with epilepsy: Cognitively compromised before the start of antiepileptic drug treatment? Epilepsia. 2010; 51:48-56.

17. Hermann B, Jones J, Sheth R, Dow C, Koehn M, Seidenberg M. Children with new-onset epilepsy: neuropsychological status and brain structure. Brain. 2006;129:2609-19.

18. Powell HW, Duncan JS. Functional magnetic resonance imaging for assessment of language and memory in clinical practice. Curr Opin Neurol. 2005;18:161-6.

19. Dupont S, Samson Y, Van de Moortele PF, Samson S, Poline JB, Hasboun D, Le Bihan D, Baulac M. Bilateral hemispheric alteration of memory processes in right medial temporal lobe epilepsy. J Neurol Neurosurg Psychiatry. 2002;73:478-85.

20. Meletti S, Benuzzi F, Nichelli P, Tassinaria CA. Damage to the right hippocampal-amygdala formation during early infancy and recognition of fearful faces: neuropsychological and $\mathrm{FMRI}$ evidence in subjects with temporal lobe epilepsy. Ann N Y Acad Sci. 2003;1000:385-8.

21. Wong SW, Jong L, Bandur D, Bihari F, Yen YF, Takahashi AM, Lee DH, Steven DA, Parrent AG, Pigott SE, Mirsattari SM. Cortical reorganization following anterior temporal lobectomy in patients with temporal lobe epilepsy. Neurology. 2009;73:518-25.

22. Weber B, Wellmer J, Schur S, Dinkelacker V, Ruhlmann J, Mormann F, Axmacher N, Elger CE, Fernandez G. Presurgical language $\mathrm{fMRI}$ in patients with drug-resistant epilepsy: effects of task performance. Epilepsia. 2006;47:880-6.

23. Thivard L, Lehericy S, Krainik A, Adam C, Dormont D, Chiras J, Baulac M, Dupont S. Diffusion tensor imaging in medial temporal lobe epilepsy with hippocampal sclerosis. Neuroimage. 2005;28:682-90. 
24. Berl MM, Balsamo LM, Xu B, Moore EN, Weinstein SL, Conry JA, Pearl PL, Sachs BC, Grandin CB, Frattali $C$, Ritter FJ, Sato S, Theodore WH, Gaillard WD. Seizure focus affects regional language networks assessed by fMRI. Neurology. 2005;65:1604-11.

25. Billingsley RL, McAndrews MP, Crawley AP, Mikulis DJ. Functional MRI of phonological and semantic processing in temporal lobe epilepsy. Brain. 2001;124:1218-27.

26. Bonelli SB, Powell RH, Yogarajah M, Samson RS, Symms MR, Thompson PJ, Koepp MJ, Duncan JS. Imaging memory in temporal lobe epilepsy: predicting the effects of temporal lobe resection. Brain. 2010;133:1186-99.

27. Mechanic-Hamilton D, Korczykowski M, Yushkevich PA, Lawler K, Pluta J, Glynn S, Tracy Jl, Wolf RL, Sperling MR, French JA, Detre JA. Hippocampal volumetry and functional MRI of memory in temporal lobe epilepsy. Epilepsy Behav. 2009;16:128-38.

28. Vannest J, Szaflarski JP, Privitera MD, Schefft BK, Holland SK. Medial temporal fMRI activation reflects memory lateralization and memory performance in patients with epilepsy. Epilepsy Behav. 2008;12:410-8.

29. Powell HW, Richardson MP, Symms MR, Boulby PA, Thompson PJ, Duncan JS, Koepp MJ. Reorganization of verbal and nonverbal memory in temporal lobe epilepsy due to unilateral hippocampal sclerosis. Epilepsia. 2007;48:1512-25.

30. Engelsen BA, Gramstad A, Thomsen T, Beneventi H, Ersland L, Smievoll Al, Lundervold A, Hugdahl K. Frontoparietal activation during delayed visuospatial recall in patients with epilepsy due to hippocampal sclerosis. Epilepsy Behav. 2006;8:565-74.

31. Dupont S, Samson Y, Van de Moortele PF, Samson S, Poline JB, Adam C, Lehericy S, Le Bihan D, Baulac M. Delayed verbal memory retrieval: a functional MRI study in epileptic patients with structural lesions of the left medial temporal lobe. Neuroimage. 2001;14:995-1003.

32. Dupont S, Van de Moortele PF, Samson S, Hasboun D, Poline JB, Adam C, Lehericy S, Le Bihan D, Samson Y, Baulac M. Episodic memory in left temporal lobe epilepsy: a functional MRI study. Brain. 2000;123:1722-32.

33. Cheung MC, Chan AS, Chan YL, Lam JM, Lam W. Effects of illness duration on memory processing of patients with temporal lobe epilepsy. Epilepsia. 2006;47:1320-8.

34. Eliassen JC, Holland SK, Szaflarski JP. Compensatory brain activation for recognition memory in patients with medication-resistant epilepsy. Epilepsy Behav. 2008;13:463-9.

35. Vlooswijk MC, Jansen JF, Reijs RP, de Krom MC, Kooi ME, Majoie HJ, Hofman PA, Backes WH, Aldenkamp AP. Cognitive $\mathrm{fMRI}$ and neuropsychological assessment in patients with secondarily generalized seizures. Clin Neurol Neurosurg. 2008;110:441-50.

36. Helmstaedter C, Kockelmann E. Cognitive outcomes in patients with chronic temporal lobe epilepsy. Epilepsia. 2006;47 Suppl 2:96-8.

37. Vlooswijk MC, Jansen JF, Majoie HJ, Hofman PA, de Krom MC, Aldenkamp AP, Backes WH. Functional connectivity and language impairment in cryptogenic localization-related epilepsy. Neurology. 2010; 75:395-402.

38. Waites AB, Briellmann RS, Saling MM, Abbott DF, Jackson GD. Functional connectivity networks are disrupted in left temporal lobe epilepsy. Ann Neurol. 2006;59:335-43.

39. Frings L, Schulze-Bonhage A, Spreer J, Wagner K. Remote effects of hippocampal damage on default network connectivity in the human brain. J Neurol. 2009;256:2021-9.

40. Calhoun VD, Liu J, Adali T. A review of group ICA for fMRI data and ICA for joint inference of imaging, genetic, and ERP data. Neuroimage. 2009;45(1 Suppl):S163-72.

41. Zhang Z, Lu G, Zhong Y, Tan Q, Liao W, Wang Z, Wang Z, Li K, Chen H, Liu Y. Altered spontaneous neuronal activity of the default-mode network in mesial temporal lobe epilepsy. Brain Res. 2010;1323:152-60.

42. Zhang Z, Lu G, Zhong Y, Tan Q, Liao W, Chen Z, Shi J, Liu Y. Impaired perceptual networks in temporal lobe epilepsy revealed by resting fMRI. J Neurol. 2009;256:1705-13.

43. Tzourio-Mazoyer N, Landeau B, Papathanassiou D, Crivello F, Etard O, Delcroix N, Mazoyer B, Joliot M. Automated anatomical labeling of activations in SPM using a macroscopic anatomical parcellation of the MNI MRI single-subject brain. Neuroimage. 2002;15:273-89.

44. Stam CJ, Reijneveld JC. Graph theoretical analysis of complex networks in the brain. Nonlinear Biomedical Physics. 2007;1:3. 
45. van den Heuvel MP, Stam CJ, Kahn RS, Hulshoff Pol HE. Efficiency of functional brain networks and intellectual performance. J Neurosci. 2009;29:7619-24.

46. Liao W, Zhang Z, Pan Z, Mantini D, Ding J, Duan X, Luo C, Lu G, Chen H. Altered functional connectivity and small-world in mesial temporal lobe epilepsy. PLoS One. 2010;5:e8525.

47. Wagner K, Frings L, Halsband U, Everts R, Buller A, Spreer J, Zentner J, Schulze-Bonhage A. Hippocampal functional connectivity reflects verbal episodic memory network integrity. Neuroreport. 2007;18:1719-23.

48. Bettus G, Guedj E, Joyeux F, Confort-Gouny S, Soulier E, Laguitton V, Cozzone PJ, Chauvel P, Ranjeva JP, Bartolomei F, Guye M. Decreased basal fMRI functional connectivity in epileptogenic networks and contralateral compensatory mechanisms. Hum Brain Mapp. 2009;30:1580-91.

49. Frings L, Schulze-Bonhage A, Spreer J, Wagner K. Reduced interhemispheric hippocampal BOLD signal coupling related to early epilepsy onset. Seizure. 2009;18:153-7.

50. Addis DR, Moscovitch M, McAndrews MP. Consequences of hippocampal damage across the autobiographical memory network in left temporal lobe epilepsy. Brain. 2007;130:2327-42.

51. Zhang Z, Lu G, Zhong Y, Tan Q, Yang Z, Liao W, Chen Z, Shi J, Liu Y. Impaired attention network in temporal lobe epilepsy: a resting fMRI study. Neurosci Lett. 2009;458:97-101.

52. Powell HW, Parker GJ, Alexander DC, Symms MR, Boulby PA, Wheeler-Kingshott CA, Barker GJ, Koepp MJ, Duncan JS. Abnormalities of language networks in temporal lobe epilepsy. Neuroimage. 2007; 36:209-21.

53. Rodrigo S, Oppenheim C, Chassoux F, Hodel J, de Vanssay A, Baudoin-Chial S, Devaux B, Meder JF. Language lateralization in temporal lobe epilepsy using functional MRI and probabilistic tractography. Epilepsia. 2008;49:1367-76.

54. Diehl B, Busch RM, Duncan JS, Piao Z, Tkach J, Luders HO. Abnormalities in diffusion tensor imaging of the uncinate fasciculus relate to reduced memory in temporal lobe epilepsy. Epilepsia. 2008;49: 1409-18.

55. Powell HW, Parker GJ, Alexander DC, Symms MR, Boulby PA, Barker GJ, Thompson PJ, Koepp MJ, Duncan JS. Imaging language pathways predicts postoperative naming deficits. J Neurol Neurosurg Psychiatry. 2008;79:327-30.

56. Flugel D, Cercignani M, Symms MR, O'Toole A, Thompson PJ, Koepp MJ, Foong J. Diffusion tensor imaging findings and their correlation with neuropsychological deficits in patients with temporal lobe epilepsy and interictal psychosis. Epilepsia. 2006;47:941-4.

57. McDonald CR, Ahmadi ME, Hagler DJ, Tecoma ES, Iragui VJ, Gharapetian L, Dale AM, Halgren E. Diffusion tensor imaging correlates of memory and language impairments in temporal lobe epilepsy. Neurology. 2008;71:1869-76.

58. van Dellen E, Douw L, Baayen JC, Heimans JJ, Ponten SC, Vandertop WP, Velis DN, Stam CJ, Reijneveld JC. Long-term effects of temporal lobe epilepsy on local neural networks: a graph theoretical analysis of corticography recordings. PloS One. 2009;4:e8081.

59. Ponten SC, Douw L, Bartolomei F, Reijneveld JC, Stam CJ. Indications for network regularization during absence seizures: weighted and unweighted graph theoretical analyses. Experimental Neurology. 2009;217:197-204.

60. Ponten SC, Bartolomei F, Stam CJ. Small-world networks and epilepsy: graph theoretical analysis of intracerebrally recorded mesial temporal lobe seizures. Clin Neurophysiol. 2007;118:918-27.

61. Rosenkranz K, Lemieux L. Present and future of simultaneous EEG-fMRI. MAGMA. 2010;23:309-16.

62. Fox MD, Raichle ME. Spontaneous fluctuations in brain activity observed with functional magnetic resonance imaging. Nat Rev Neurosci. 2007;8:700-11.

63. Auer DP. Spontaneous low-frequency blood oxygenation level-dependent fluctuations and functional connectivity analysis of the 'resting' brain. Magnetic Resonance Imaging. 2008;26:1055-64.

64. Jokeit $\mathrm{H}$, Ebner $\mathrm{A}$. Long term eff ects of refractory temporal lobe epilepsy on cognitive abilities: a cross sectional study. J Neurol Neurosurg Psychiatry. 1999;67:4-50. 


\section{Chapter}

Cognitive fMRI and neuropsychological assessment in patients with secondarily generalized seizures

MCG Vlooswijk, JFA Jansen, RP Reijs, MCTFM de Krom, ME Kooi, HJM Majoie, PAM Hofman, WH Backes, AP Aldenkamp Clinical Neurology and Neurosurgery 2008;110(5):441-450 


\section{Abstract}

\section{Objectives}

Cognitive dysfunction is a frequent comorbid disorder in epilepsy which has been associated with high seizure frequency. We examined the effect of secondarily generalized tonic-clonic seizures (SGTCS) on cognitive dysfunction using neuropsychological assessment and functional MRI.

\section{Patients and methods}

Sixteen patients with localization-related epilepsy of varying etiologies and SGTCS underwent extensive neuropsychological assessment. Functional MRI was performed probing the frontal and temporal lobes with two paradigms aimed at investigating speed of mental processing and working memory.

\section{Results}

A high number of total life-time SGTCS was associated with lower intelligence scores. Moreover, a trend towards cognitive decline related to the number of SGTCS was observed. A relatively increased prefrontal activation related to the number of SGTCS was demonstrated, plus a trend towards a decreased activation in the frontotemporal areas.

\section{Conclusion}

High numbers of SGTCS are associated with a drop in intelligence scores and altered prefrontal brain activation. A shift from frontotemporal to prefrontal activation seems to have occurred, suggesting that a functional reorganization of working memory is induced by a high number of SGTCS. It remains uncertain if this reorganization reflects a compensation mechanism, or the underlying pathological processes of cognitive dysfunction. 


\section{Introduction}

In clinical experience, patients with persistent seizures are prone to deteriorate cognitively. Cognitive impairment is in fact the most frequent comorbid disorder in epilepsy. ${ }^{1,2}$ The spectrum of cognitive problems in epilepsy ranges from the frequently reported memory deficits and mental slowing, to sometimes even global cognitive deterioration. Many factors have been proposed to contribute to cognitive impairment, such as the underlying disease causing epilepsy, cognitive side-effects of antiepileptic drugs (AED), ${ }^{3,4}$ seizure-induced head trauma, ${ }^{5}$ and ongoing interictal epileptic brain activity. ${ }^{6}$ Cognitive decline has been associated with status epilepticus as well. ${ }^{7}$ Also single seizures, especially secondarily generalized seizures, are thought to have an effect on cognition. In various studies, changes in metabolism ${ }^{8-11}$ and neuronal loss ${ }^{12}$ were observed after secondarily generalized tonic-clonic seizures (SGTCS). In patients with a high number of SGTCS, cognitive deterioration has also been described, ${ }^{5,7,13,14}$ although data are sometimes contradictory. ${ }^{15}$

Different hypotheses are proposed for the mechanisms by which such seizure-induced cognitive deterioration could be produced. In animal models, seizures cause changes in protein expression, protein modification, mossy fiber sprouting and synaptic reorganization. ${ }^{16}$ Above that, cell loss in varying regions of the limbic system due to necrosis and apoptosis induced by seizures is noticed. ${ }^{17}$ It remains unclear, however, if these observed changes occur in either a serial or a parallel fashion ${ }^{18}$ and whether they are relevant for deterioration in patients with epilepsy.

We are interested in the association between SGTCS and cognitive dysfunction. Considering the global cognitive problems observed in patients with SGTCS, which are often accompanied by mental slowing and impairment in executive function, ${ }^{19}$ it is likely to assume that the frontal lobe is involved in this cognitive dysfunction. The involvement of the frontal lobes could be explained by the secondary generalization of seizures. Therefore, the neuronal correlate for cognitive dysfunction in patients with SGTCS might be located in the frontal or prefrontal regions.

Previous studies have investigated possible structural alterations associated with seizure-induced cognitive dysfunction. There have been no studies with conventional MRI which could demonstrate a relation between cognitive dysfunction and structural alterations in epilepsy. With volumetry, a more advanced technique, associations between volume reductions of the hippocampus, ${ }^{20}$ cerebellum, ${ }^{21}$ and global cerebral volume reduction ${ }^{22}$ and memory deficits as well as poor cognitive performance have been demonstrated. Yet, another study could not establish an association between structural changes and executive function. ${ }^{23}$ However, functional changes might occur in an earlier stage than structural alterations and are possibly easier identified in epilepsy, which is a functional rather than structural disorder of the brain. Therefore, 
functional MRI could possibly provide a model for the development of cognitive dysfunction.

Functional MRI ( $\mathrm{fMRI}$ ) enables the localization of brain activation by demonstrating a blood oxygenation level-dependent (BOLD) signal during a (cognitive) paradigm. With task-related fMRI, overall differences in activation patterns between groups can be detected and localized. As the primary seizure focus and the spreading of discharges may vary strongly among patients, it is a priori unknown how seizures may alter the brain activation pattern. Ideally, one would, therefore, be able to investigate all parts of the brain, rather than only certain regions specifically related to a particular cognitive task. To overcome this problem, we propose to combine different cognitive paradigms which are known to activate different regions of the frontal and temporal lobe in healthy subjects.

Our goal was to investigate the effect of the number of SGTCS accumulated over life on the outcomes of neuropsychological testing and on the distribution of brain activation in the temporal and frontal lobes and foremost whether they are relevant for dysfunction.

\section{Methods}

\section{Participants}

The study population included 16 patients (10 women and 6 men; mean age 40.4 years; range 21-59). All patients were consecutively included from the outpatient clinics for neurology of the University Hospital Maastricht. All subjects gave informed consent and approval for the study by the local Medical Ethical Committee was obtained. Inclusion criteria for the study were: localization-related epilepsy with secondarily generalized seizures, no history of status epilepticus and no other underlying disease that could possibly cause cognitive decline. The following patient data were collected: age at onset, total number of SGTCS experienced during lifetime, partial seizure frequency per month (averaged over the last 6 months), seizure focus, etiology and drug load. Total number of SGTCS was calculated according to patient history and seizure diaries. For those patients with relatively low numbers of SGTCS, the exact number of SGTCS could be withdrawn from the patient's history. For those with relatively high numbers of SGTCS, the number was calculated according to the seizure frequency during subsequent periods, taking into account changes in seizure frequency (for example, weekly seizures during a few months followed by a period of seizure-freedom). Hence, when a higher number of SGTCS had been experienced, the total number is an approximation rather than an absolute number. Drug load was calculated by standardizing the doses of antiepileptic drugs using the 
ratio of prescribed daily dose to defined daily dose. ${ }^{24}$ No SGTCS were reported in the last 2 weeks before MRI. Patient characteristics are listed in Table 3.1.

Table 3.1 Patient characteristics.

\begin{tabular}{|c|c|c|c|c|c|c|c|c|c|}
\hline Patient & $\begin{array}{l}\text { Age } \\
\text { (y) }\end{array}$ & Sex & $\begin{array}{c}\text { Epilepsy } \\
\text { duration (y) }\end{array}$ & $\begin{array}{l}\text { Seizure } \\
\text { focus }^{*}\end{array}$ & Etiology & $\begin{array}{l}\text { Total no. } \\
\text { SGTCS }\end{array}$ & $\begin{array}{l}\text { Partial seizure } \\
\text { frequency (per } \\
\text { month) }\end{array}$ & AED & $\begin{array}{l}\text { Drug } \\
\text { load }\end{array}$ \\
\hline 1 & 23 & $\mathrm{~F}$ & 1 & LF & $C D$ & 32 & 75 & LEV & 1.0 \\
\hline 2 & 55 & $\mathrm{~F}$ & 39 & Multiple & MTS & 1 & 75 & $\mathrm{CBZ}$ & 1.2 \\
\hline 3 & 37 & $\mathrm{~F}$ & 14 & $\mathrm{LT}$ & PT & 4 & 2 & $\mathrm{CBZ}$ & 0.6 \\
\hline 4 & 24 & $\mathrm{~F}$ & 15 & RF & crypt & 21 & 0 & $\begin{array}{l}\text { CBZ, LEV, } \\
\text { LTG }\end{array}$ & 3.0 \\
\hline 5 & 59 & $M$ & 40 & $\mathrm{LF}$ & $C D$ & 14 & 0 & LEV & 1.6 \\
\hline 6 & 56 & $\mathrm{~F}$ & 9 & Multiple & crypt & 72 & 20 & $\begin{array}{l}\text { CBZ, TPM, } \\
\text { VPA, CLB }\end{array}$ & 3.7 \\
\hline 7 & 41 & $\mathrm{~F}$ & 2 & LF & APS & 4 & 0 & $\mathrm{CBZ}$ & 1.6 \\
\hline 8 & 24 & $M$ & 17 & LT & crypt & 200 & 1 & CBZ, LEV & 2.0 \\
\hline 9 & 31 & $M$ & 27 & RT & crypt & 96 & 0 & TPM, OXC & 4.6 \\
\hline 10 & 55 & $\mathrm{~F}$ & 17 & LT & $C D$ & 22 & 0 & OXC & 1.5 \\
\hline 11 & 40 & $\mathrm{~F}$ & 34 & LT & $\begin{array}{l}\text { MTS, } \\
\text { LTL }\end{array}$ & 30 & 12 & $\begin{array}{l}\text { CBZ, LEV, } \\
\text { CZP }\end{array}$ & 1.7 \\
\hline 12 & 49 & $\mathrm{~F}$ & 44 & RT & crypt & 12 & 300 & None & 0.0 \\
\hline 13 & 57 & $M$ & 14 & Unknown & crypt & 2 & 0 & CBZ & 0.8 \\
\hline 14 & 49 & $\mathrm{~F}$ & 3 & RT & crypt & 6 & 0 & None & 0.0 \\
\hline 15 & 25 & $M$ & 10 & Unknown & $\begin{array}{l}\text { crypt } \\
\text { (AC) }\end{array}$ & 21 & 0 & OXC & 2.4 \\
\hline 16 & 21 & $M$ & 9 & LT & $\begin{array}{l}\text { crypt } \\
\text { (AC) }\end{array}$ & 2 & 0 & LEV, LTG & 2.6 \\
\hline Median & 40.5 & & 14.5 & & & 17.5 & 0 & & 1.6 \\
\hline Mean & 40.4 & & 18.4 & & & 33.7 & 28.5 & & 1.8 \\
\hline
\end{tabular}

$\mathrm{Y}=$ years; SGTCS = secondarily generalized tonic-clonic seizures; $\mathrm{F}=$ female; $\mathrm{M}=$ male; $\mathrm{LF}=$ left frontal; $\mathrm{RF}=$ right frontal; $\mathrm{LT}$ = left temporal; $\mathrm{RT}=$ right temporal; $\mathrm{CD}=$ cortical dysplasia; $\mathrm{PT}$ = post-traumatic lesion; $\mathrm{MTS}$ = mesiotemporal sclerosis; crypt, cryptogenic; $A P S=$ anti-phospholipid syndrome; LTL = left temporal lobectomy; $\mathrm{AC}=$ arachnoid cyst; $\mathrm{CBZ}=$ carbamazepine; $\mathrm{CLB}=$ clobazam; $\mathrm{CZP}=$ clonazepam; $\mathrm{LEV}=$ levetiracetam; LTG = lamotrigine; OXC = oxcarbazepine; TPM = topiramate.

${ }^{*}$ Based on the electroencephalogram.

\section{Neuropsychological testing}

All subjects underwent extensive neuropsychological testing, including tests for intelligence, handedness, attentional functions and information processing and memory function. In order to test intelligence, the Wechsler Adult Intelligence Scale third edition (WAIS-III) was used. For handedness, the Annett Handedness Questionnaire was administered. Tests reflecting speed of information processing were the Computerized Visual Searching task (CVST) and the Binary Choice Reaction Test (BCRT). With the CVST, subjects are asked to compare a centered grid pattern with 24 surrounding patterns, one of which is identical to the target pattern. The test includes 24 trials and the outcome measure is the average search time in seconds. The 
BCRT is a decision task in which the subject has to react in a different way to a red square presented on the left side of the screen than to a green square on the right side. The outcome measure is the reaction time in milliseconds. Memory function was assessed by the computerized task of recognition of words and figures. Six words and four figures are presented with a presentation time of $1 \mathrm{~s}$ per item. After a delay of $2 \mathrm{~s}$, the screen shows one of these words or figures between distractors. Subjects are asked to identify the target item. The outcome measure is the number of correct answers out of 24. CVST, BCRT and the memory tests are parts of the FePsy neuropsychological test battery. ${ }^{25}$ Based on the results of these tests combined, a composite score was derived, comparing the actual level with the expected level, based on premorbid educational level. The resulting score has five categories: (1) no intellectual decline and no cognitive dysfunction, (2) no intellectual decline but signs of mental slowing, (3) no intellectual decline but signs of dysfunction in only one area of higher cognitive functioning (i.e., language or memory), (4) no intellectual decline and two impaired areas of higher cognitive function (i.e., impaired language and memory) with or without mental slowing and (5) global intellectual decline. This 'deterioration score' is a measure for the increasing levels of cognitive deterioration starting with mental slowing, followed by difficulties in higher cognitive functioning, and eventually is displayed in lower IQ measures.

\section{fMRI data acquisition}

MR imaging was performed on a 1.5 Tesla unit (Philips Intera, Philips Medical Systems, Best, the Netherlands). Functional MRI data were acquired using a whole-brain singleshot three-dimensional (3D) blood oxygen level-dependent echo-planar imaging sequence, with repetition time $2 \mathrm{~s}$, echo time $50 \mathrm{~ms}$, flip angle $90^{\circ}$, voxel size $3.5 \mathrm{~mm} \times 3.5 \mathrm{~mm} \times 3.5 \mathrm{~mm}$, matrix $64 \times 64,34$ contiguous slices per volume, and 96 volumes per acquisition. For anatomical reference, we acquired a 3D T1-weighted fast field-echo image, with repetition time $11 \mathrm{~ms}$, echo time $3.5 \mathrm{~ms}$, flip angle $90^{\circ}$, matrix $256 \times 256,150$ contiguous slices, and voxel size $3.5 \mathrm{~mm} \times 3.5 \mathrm{~mm} \times 3.5 \mathrm{~mm}$.

\section{fMRI activation paradigms}

Two experimental block paradigms were used. The Sternberg letter recognition paradigm is a choice reaction test reflecting working memory performance. ${ }^{26}$ Letters were visually presented to the patients to maintain in working memory. Subjects then responded to the presentation of single letters by pressing a button with either their right or left hand to indicate whether or not the letter was in the memorized set of letters. In the baseline condition, subjects were asked to focus on a cross-hair. The paradigm consisted of 16 blocks (memory set for $4 \mathrm{~s}$ followed by 13 response letters of $2 \mathrm{~s}$ each) alternating with 16 baseline rest condition blocks (30 s each). The memory set varied from 1 to 4 letters, presented in a random fashion with a total of four blocks for each load. Prior to the actual fMRI scanning, the task was practiced within 
the scanner with one block. Monitoring of the button pressing output during this practice indicated good performance. The contrast between activation in baseline condition and all loads was used for further analysis. In previous studies, prefrontal and temporal areas were activated by this paradigm. ${ }^{27}$

The second paradigm was a covert variation of the Stroop test. ${ }^{28}$ Words of colour names were presented displayed in a different colour than the colour it actually named or in the same colour. Subjects were instructed to think of the colour in which the word was displayed. For example, the word blue was written in red letters, so the subject had to think 'red'. In the baseline condition, subjects were asked to focus on a cross-hair. This task reflects self-regulatory control processes by asking subjects to inhibit a more automatic behaviour (reading a word) to perform a less automatic one (naming of a colour). Usually, activation of the lateral prefrontal area during the Stroop paradigm is observed in healthy subjects. The paradigm comprised of six activation blocks (15 words, $2 \mathrm{~s}$ each) alternating with six baseline rest condition blocks (30 s each). Since the performance during the Sternberg paradigm was confirmed to be proper, and as the task difficulty of both Sternberg and Stroop is similar, a normal performance during the Stroop paradigm was assumed. The contrast between baseline condition and activation blocks was used for analysis. With this paradigm, activation of prefrontal areas, especially the inferior lateral prefrontal cortex, has been demonstrated in healthy adults. ${ }^{29}$

\section{Image analysis}

Statistical analysis was performed in MATLAB (Mathworks, Natick, Massachusetts, USA) using brain activation contrasts (between task performance and baseline) according to the General Linear Model (GLM) as implemented in the statistical parametric mapping (SPM) software package (SPM2) (Wellcome Department of Cognitive Neurology, London, UK). The BOLD images were realigned intra-individually to the first image in each time-series on a voxel-by-voxel basis to correct for head movement. Realigned fMRI images were transformed into the standardized stereotactic reference system developed by the Montreal Neurological Institute based on the Talairach and Tournoux atlas ${ }^{30}$ and smoothed with a 6-mm Gaussian kernel. In the GLM, the standard discrete cosine-set was used to correct for nuisance (lowfrequency). Additionally, the time-series were convolved with the hemodynamic response function.

\section{Statistical analysis}

Areas with statistically significant changes in signal intensity were determined on a voxel-by-voxel basis using the Student's $t$-test. Activation is mostly dependent on the statistical threshold used to identify active voxels. The exact location of activated voxels during a certain paradigm may vary among different subjects. Therefore, one 
should take into account this inter-individual functional variability, especially in small subject groups. ${ }^{31}$ Analyzing brain activation using a voxel-wise method may not be suitable to investigate changes in activation patterns in the current patient group, which is characterized by a high heterogeneity of etiology. This heterogeneity may cause an increased inter-individual variability of activation of individual voxels. Although the location of activated voxels within all subjects may not match precisely, it is still possible to obtain relevant information on activation on a regional level. Therefore, we did not use one fixed statistical threshold for all subjects, but set the level of significance in each subject so that it corresponded to a fixed number of activated voxels. This method has previously been successfully applied by Knecht et al. $^{32}$ We selected for every patient the 5000 most active voxels, which is approximately $1 \%$ of the brain, in a predefined region using masks created with WFUPickatlas. ${ }^{33}$ Further analyses were performed on those 5000 most active voxels. The predefined regions consisted of the prefrontal area (medial frontal gyrus, gyrus rectus, orbital gyrus, superior frontal gyrus, middle frontal gyrus, inferior frontal gyrus, subcallosal gyrus), frontotemporal area (superior temporal gyrus, transverse temporal gyrus, insula and part of the inferior frontal gyrus adjacent to the insula and temporal lobe), temporal area (middle temporal gyrus, mesiotemporal cortex, inferior temporal gyrus), cingulate cortex, Broca's area (Brodmann areas 44 and 45 in the inferior frontal gyrus) and Wernicke's area (Brodmann areas 22, 39 and 40). Subsequently, the relative activation level (i.e., the proportion of more-significant voxels) in these regions was calculated (expressed as the fraction of the 5000 active voxels from the whole predefined region within the specific region) in order to detect increased or decreased activity reflecting hyper- or hypoactivation, respectively. Clinical and neuropsychological data analyses were performed in SPSS for Windows (Rel. 11.5.0. 2002. Chicago: SPSS Inc.). Correlations of fMRI data, epilepsy parameters, and neuropsychological parameters were calculated with parametric (Pearson correlation) and non-parametric (Spearman correlation) statistical tools. As the total number of SGTCS is rather an approximation than an absolute number, we used non-parametric Spearman correlation when analyzing neuropsychological and $\mathrm{fMRI}$ data related to the number of SGTCS. This analysis is based on the ranking of the patients with respect to the number of total SGTCS rather than on the influence of the absolute amount of seizures.

Multiple end point testing was controlled for by first investigating in what regions differences in $\mathrm{fMRI}$ activation appeared. To this end, the activation maps from the Sternberg and Stroop fMRI paradigms were combined per region according to the method of O'Brien-Läuter. ${ }^{34,35}$ In this way, a more global impression of prefrontal and frontotemporal activation was obtained. For the combined analysis, statistical significance was calculated with two-tailed Student's $t$-tests with Hochberg correction for multiple (6 regions of interest) comparisons. ${ }^{36}$ 


\section{Results}

\section{Patient characteristics}

Patient characteristics are described in Table 3.1. Structural MRI findings are summarized in Table 3.2.

Table 3.2 Neuroradiological structural MRI findings.

\begin{tabular}{ll}
\hline Patient & \\
1 & Cortical dysplasia left frontotemporal region \\
2 & Non-specific white matter lesions, right hippocampal atrophy \\
3 & Left temporal lobe atrophy and contusion extending to the hippocampus and frontal lobe \\
4 & No cerebral abnormalities \\
5 & Cortical dysplasia left temporal lobe \\
6 & Non-specific white matter lesions \\
7 & Diffuse white matter and subcortical lesions \\
8 & No cerebral abnormalities \\
9 & Left hippocampal atrophy \\
10 & Cortical dysplasia left temporal and right parietal, lacunar infarction left lentiform nucleus and \\
11 & right caudate nucleus, global cerebral atrophy \\
12 & Resection of anterior part of left hippocampus and partial resection of left amygdala \\
13 & Left temporal pole atrophy \\
14 & Non-specific white matter lesions, lacunar infarction in the left superior frontal gyrus \\
15 & Post-traumatic gliosis left temporal lobe \\
16 & Arachnoid cyst left temporal pole \\
\hline
\end{tabular}

\section{Neuropsychological assessment}

Neuropsychological assessment measures are summarized in Table 3.3.

\section{Correlation of neuropsychological findings with SGTCS}

Subjects with a higher number of total SGTCS had significantly lower IQ measures (total IQ: $p=0.01$; verbal IQ: $p=0.01$ and performance IQ: $p=0.01$ ) than those with a lower amount of SGTCS). A trend towards higher deterioration scores was noticed with a higher number of SGTCS $(p=0.07)$. No correlation was found between the remainder of neuropsychological tests and number of SGTCS experienced. 
Table 3.3 Neuropsychological assessment measures.

\begin{tabular}{|c|c|c|c|c|c|c|c|c|}
\hline Patient & $\begin{array}{c}\text { Total } \\
\text { IQ }\end{array}$ & Verbal IQ & Performance IQ & $\begin{array}{l}\text { Deterioration } \\
\text { score }\end{array}$ & $\begin{array}{l}\text { Word } \\
\text { recognition* }\end{array}$ & $\begin{array}{c}\text { Figure } \\
\text { recognition* }\end{array}$ & $\begin{array}{c}\text { BCRT- } \\
\text { mean RT } \\
(\mathrm{ms})\end{array}$ & $\begin{array}{c}\text { CVST- } \\
\text { mean RT } \\
\text { (s) }\end{array}$ \\
\hline 1 & 92 & 96 & 90 & 4 & 14 & NA & 364 & 9.3 \\
\hline 2 & 135 & 136 & 126 & 2 & 21 & NA & 495 & 12.8 \\
\hline 3 & 131 & 124 & 134 & 4 & 22 & 6 & 499 & 9.5 \\
\hline 4 & 101 & 97 & 107 & 4 & 4 & 11 & 571 & 19.7 \\
\hline 5 & 102 & 100 & 104 & 3 & 15 & 10 & 452 & 22.0 \\
\hline 6 & 86 & 85 & 90 & 5 & 12 & 7 & 746 & 26.4 \\
\hline 7 & 126 & 122 & 126 & 3 & 17 & 7 & 413 & 10.2 \\
\hline 8 & 112 & 110 & 112 & 4 & 15 & 11 & 387 & 11.0 \\
\hline 9 & 67 & 68 & 74 & 3 & 14 & 6 & 390 & 18.1 \\
\hline 10 & 65 & 66 & 70 & 2 & 18 & 9 & 1799 & 18.9 \\
\hline 11 & 120 & 124 & 112 & 1 & 20 & 13 & 444 & 12.4 \\
\hline 12 & 112 & 111 & 112 & 1 & 20 & 9 & 416 & 15.3 \\
\hline 13 & 115 & 113 & 116 & 1 & 18 & 13 & 395 & 13.4 \\
\hline 14 & 124 & 119 & 126 & 1 & 18 & 13 & 469 & 10.1 \\
\hline 15 & 111 & 113 & 107 & 1 & 21 & 13 & 419 & 11.0 \\
\hline 16 & 101 & 103 & 98 & 1 & 19 & 16 & 401 & 11.7 \\
\hline Median & 112 & 111 & 110 & 2.5 & & & & \\
\hline Mean(SD) & $106(18)$ & $105(20)$ & $107(18)$ & & $17(5)$ & $21(30)$ & $541(348)$ & $14.5(5.1)$ \\
\hline
\end{tabular}

$\mathrm{IQ}=$ intelligence quotient; $\mathrm{RT}$ = reaction time; $\mathrm{BCRT}=$ binary choice reaction test; CVST = computerized visual searching task; $\mathrm{NA}=$ not available, *absolute score (maximum score 24); $\mathrm{ms}=$ milliseconds; $\mathrm{s}=$ seconds; SD = standard deviation.

\section{fMRI}

fMRI activation maps were analyzed using predefined brain regions. Activation patterns in these regions were compared to the number of SGTCS experienced.

A global impression of frontal and temporal activation was obtained by combining the $Z$-values for both paradigms, which demonstrated a significantly higher activation in the prefrontal region $(p<0.01, r=0.65)$ (Figure 3.3) and the cingulate region $(p=0.02$, $r=0.57)$ in relation to a higher number of SGTCS. A trend towards lower activation in the frontotemporal region was observed $(p=0.05 ; r=0.49)$.

A detailed analysis of both paradigms separately is illustrated hereafter. In the prefrontal regions, a higher activation could be observed during the Stroop paradigm ( $p=0.01, r=0.60$ ) with increasing number of SGTCS experienced. For both Stroop and Sternberg paradigms, no significant correlation was found between activation patterns and number of SGTCS in the frontotemporal region, the temporal regions and Broca's area, $(p>0.05)$. In the cingulate cortex, higher activation was seen in the Stroop paradigm $(p<0.01 ; r=0.72)$ but not in the Sternberg paradigm $(p>0.05)$. Dividing the cingulate cortex in an anterior and a posterior part, no statistically significant association was found in Stroop $(p>0.05)$. 
We then compared the results of the global deterioration scale with prefrontal activation patterns. No statistically significant relation could be demonstrated ( $p=0.24$ and $p=0.19$ for Stroop and Sternberg, respectively), but data showed a trend towards higher activation in the prefrontal region for higher deterioration scores ( $r=0.46$ for Stroop and $r=0.35$ for Sternberg) (Figure 3.1). Figure 3.2 shows an example of brain activation in the Sternberg paradigm of patient 3 with 4 life-time SGTCS compared to patient 6 with 72 life-time seizures.

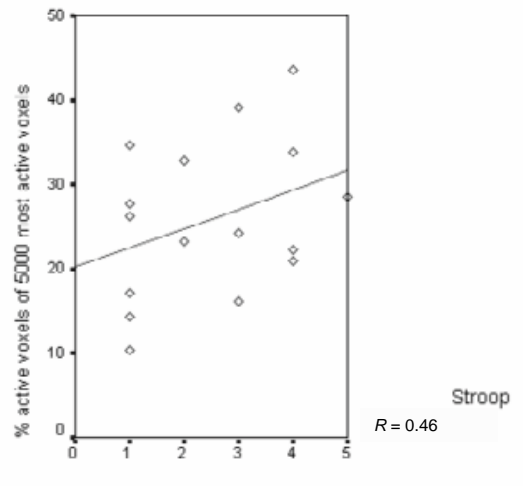

Deterinration score

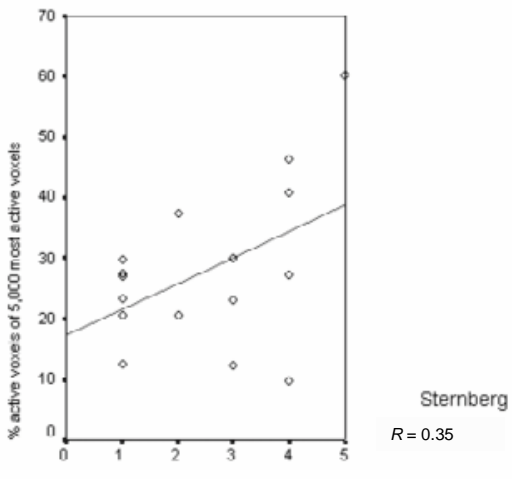

Detenoration score

Figure 3.1 Relative prefrontal activation patterns for Stroop (left) and Sternberg (right) paradigms in relation to cognitive deterioration scores.

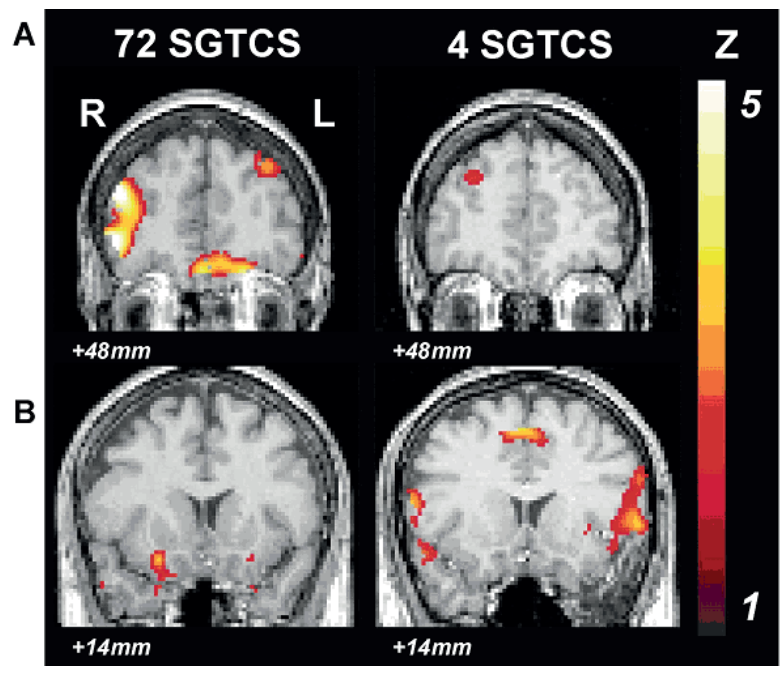

Figure 3.2 Coronal images of fMRI activation maps, obtained for the Sternberg paradigm overlaid on a normalized T1-weighted MR image, with left a typical patient with many SGTCS, and right a patient with few SGTCS. (A) Prefrontal region, (B) frontotemporal region. Slice positions are $y=+8 \mathrm{~mm}$ in stereotactic Talairach coordinates. 


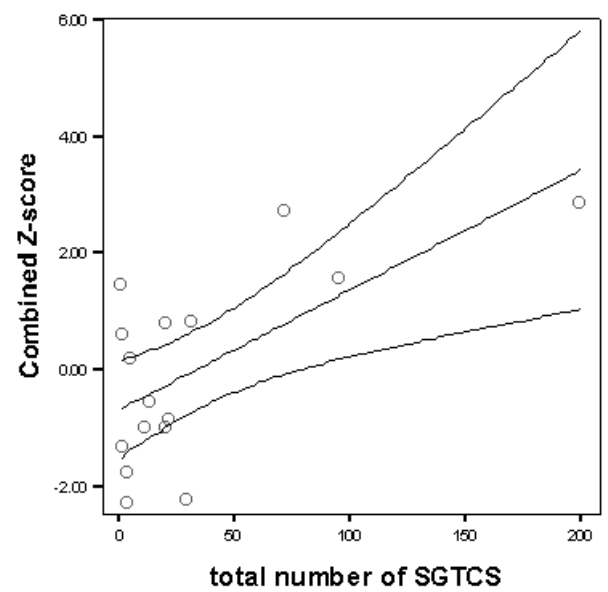

Figure 3.3 Relative prefrontal activation for Sternberg and Stroop combined in relation to total number of SGTCS.

In a post-hoc analysis, we focused on the prefrontal lobe by looking at the different Brodmann areas to specify the localization of the elevated activation related to a higher number of SGTCS. In the Stroop paradigm, a significantly elevated activation pattern was seen in the rostral part of the superior and the middle frontal gyri and the gyrus rectus (Brodmann areas 10, $p=0.04$; and 11, $p=0.03$ ) of the right hemisphere.

\section{Discussion}

In this study, a statistically significant correlation between number of SGTCS experienced during life-time, and intellectual functioning as measured by the WAIS was observed. Hence, there is an evident association between a high number of seizures and cognitive dysfunction. In order to investigate whether patients with cognitive dysfunction show a progressive decline, we assessed deterioration using the deterioration score, a more global observation of cognitive functioning. This score showed a tendency to increased deterioration with a higher number of SGTCS. Possibly, due to the limited number of patients this study lacks the power to reach a statistically significant association between number of SGTCS and deterioration score. Naturally, an ideal measure for cognitive deterioration would be to compare cognitive tests with a test-retest interval of at least some years. However, we consider the comparison of the obtained neuropsychological measures with the expected level of cognitive functioning a reasonable alternative. This is confirmed by the fact that both outcomes, intellectual functioning and the deterioration score, show a trend toward the same direction: an association between number of SGTCS and cognitive 
deterioration which is in line with previous studies. ${ }^{7,13,14,19,37,38}$ Interestingly, some patients with a small number of SGTCS demonstrated cognitive deterioration whereas others with a higher number showed no signs of cognitive deterioration. Therefore, it is difficult to predict cognitive deterioration due to frequent seizures on an individual patient level. Currently, a study is being performed to investigate this apparent difference in vulnerability to seizures in a larger patient population and to determine the clinical factors involved therein.

Moreover, we have demonstrated a relatively increased prefrontal activation related to the number of SGTCS. Furthermore, a trend towards a decreased activation in the frontotemporal areas was observed. Both paradigms used in the current study are aimed at indicating activation of the brain areas involved in verbal working memory and speed of mental processing, more specifically, the temporal areas and the prefrontal area, especially the dorsolateral prefrontal cortex. The relative increase of activation in the prefrontal region and decrease in frontotemporal areas, could be interpreted as a shift of activation from frontotemporal to prefrontal areas. This suggests that a functional reorganization of working memory has taken place in patients with a higher number of SGTCS.

We used the combination of two cognitive tasks to investigate the frontal and temporal lobe to detect differences in brain activation. Since the cortical spreading of seizures of varying primary seizure foci may differ strongly between patients, it was a priori unlikely to localize alterations in brain activation. Consequently, using only a single cognitive task would show different changes in brain activation patterns between epilepsy patients. Combining multiple cognitive paradigms facilitates this spatial variability and allows for a more sensitive detection of cerebral activation changes.

Previous studies have suggested different hypotheses for cerebral reorganization. It is commonly hypothesized that functional reorganization is an effect of cerebral plasticity, particularly in temporal lobe epilepsy. ${ }^{16,18}$ Seizures are thought to cause a cascade of molecular and genomic changes, which may be relevant for the morphologic and functional long-term changes noticed in epilepsy. ${ }^{39}$ It is thought that neurogenesis might underlie functional reorganization by developing new neuronal circuits. ${ }^{40}$ These circuits can have a two-fold effect: (i) new circuits could disrupt important existing neuronal pathways, thereby interfering with normal learning and memory; ${ }^{40}$ (ii) new neuronal pathways could bypass pathological circuits and have a beneficial effect. Especially in patients with early-onset localization-related epilepsies, reorganization of language and verbal memory has been suggested as an explanation for the observed increased recruitment of the homologous contralateral language areas. $^{41,42}$ 
There are two possible interpretations for the changes in activation pattern often noticed in patients with epilepsy. First, it could be a reflection of a compensatory mechanism aimed at the preservation of normal cognitive function. In patients with temporal lobe epilepsy, language reorganization due to early left temporal damage (before age of three), was not associated with verbal memory deficits. In older subjects, however, language reorganization concurred with verbal memory deficits. ${ }^{43}$ Some fMRI studies have shown marked alterations in activation patterns without altered cognitive functioning. In a study of patients with left temporal lobe epilepsy, higher interictal spiking frequency was associated with more pronounced right-sided frontal activity during speech $\mathrm{fMRI}$, whereas patients performed equally as controls on a word fluency task. ${ }^{44}$

Alternatively, altered activation could also reflect the pathological processes underlying cognitive deterioration. Studies supporting this theory show cognitive dysfunction associated with altered activation patterns. In patients with right temporal seizure focus and left language dominance, activation of the homologous language areas correlated inversely with verbal memory performance. ${ }^{42}$

Whether or not the functional reorganization demonstrated in the current study is efficient to maintain a normal functioning level, remains uncertain. Neuropsychological measures indicative of mental slowing and working memory were not significantly different for patients with a high or low number of SGTCS. Nevertheless, as mentioned before, the deterioration score did show an important trend towards more cognitive deficits with a high number of SGTCS. This suggests that the functional reorganization might not be sufficient to maintain normal cognitive functioning and compensate for cognitive deficits caused by SGTCS.

The prefrontal changes observed in our study are in accordance with previous studies. In patients with depression, a similar study with fMRI and a working memory paradigm showed greater activation of the lateral prefrontal cortex and the anterior cingulate compared to healthy subjects which suggested that depressed patients needed greater activation within the same neural network to maintain a similar level of performance. ${ }^{45}$ An fMRI study of phonological and semantic processing in left TLE patients showed increased activation in prefrontal areas without differences in accuracy compared to controls. ${ }^{46}$ Patients with mesial temporal lobe epilepsy (MTLE) who performed a verbal memory task, showed extensive activation of the left prefrontal region which was not found in healthy controls. Memory performance of patients was poor, suggesting that the altered activation reflected a dysfunction of cerebral memory processing. ${ }^{47}$ Contrarily, a recent study showed an effective shift of spatial and figural memory to more posterior cerebral parts in patients with MTLE explained in part by a relative frontal hypoactivation. ${ }^{48}$ 
The possibility exists that the demonstrated differences in $\mathrm{FMRI}$ activation in patients with higher number of experienced SGTCS are directly related to the lower intelligence scores. It is difficult to separate the effect of SGTCS on fMRI activation patterns on the one hand and cognitive functioning on the other hand. Moreover, cognitive dysfunction may result in the usage of different cognitive mechanisms when performing an fMRI task, which might be reflected in altered fMRI activation patterns. Frontal lobe activation may reflect a compensation mechanism for deficits in working memory rehearsal pathways involving temporal lobe and non-frontal processes. In any case, the altered $\mathrm{fMRI}$ activation patterns as demonstrated in this study are not a consequence of poor performance during both paradigms in patients with low IQ scores because monitoring of the pressed buttons showed good performance.

This study has several limitations. The patient group studied did not exhibit a homogeneously distributed range of total number of generalized seizures. Due to the limited number of patients, the data lack sufficient power to determine a threshold effect. We have re-analyzed the data of frontal lobe activation of the combined Stroop and Sternberg paradigms with exclusion of the patient with 200 SGTCS, showing no statistically significant effect $(p=0.20$, Spearman correlation). However, nonparametric Spearman correlation is applied, based on the ranking of the patients with respect to the number of total SGTCS rather than on the influence of the absolute amount of seizures which does not require normal data distribution. In other words, the exact number of 200 SGTCS is not quantitatively weighted as 200 , but qualitatively as the highest number of SGTCS. Therefore, we feel this patient should be included in the analysis.

Additionally, the limited number of patients complicates the applicability of our results. However, even in this small population of patients with varying etiologies and seizure foci, we have been able to demonstrate a statistically significant effect of number of SGTCS on prefrontal activation patterns. Moreover, SGTCS are associated with intellectual functioning and probably are an important factor in cognitive decline. However, a more homogeneous and larger epilepsy population is required to confirm our results. Furthermore, it remains to be elucidated whether there is a causal relation between SGTCS and intellectual functioning or whether a high number of SGTCS and low IQ are both a reflection of the severity of the underlying epilepsy pathology such as cortical or other cerebral abnormalities. The latter would imply that aggressive seizure treatment would not improve cognitive outcome.

The cross-sectional design of this study lacks the ability to show cognitive decline or progressive changes in activation patterns. In the future, prospective studies with test and retest intervals of a few years investigating progression of cognitive dysfunction and cerebral activation changes are needed. 
To conclude, secondarily generalized seizures in frontal and temporal lobe epilepsy are associated with cognitive dysfunction. Moreover, secondarily generalized seizures are related to a relatively increased activation in the prefrontal areas as demonstrated by $\mathrm{fMRI}$ using two paradigms aimed at activating regions involved in verbal working memory and speed of mental processing. It is hypothesized that a mechanism of functional reorganization underlies the change in activation patterns but does not seem to suffice in compensating for cognitive deficits caused by SGTCS. Although in some cases cognitive deficits may be directly related to the underlying pathology of both intractable seizures and cognitive dysfunction, it is suggested that cognitive dysfunction might be prevented by proactively preventing seizures with AED or epilepsy surgery. 


\section{References}

1. Thompson PJ, Corcoran R. Everyday memory failures in people with epilepsy. Epilepsia. 1992;33 Suppl 6:S18-20.

2. Helmstaedter C. Effects of chronic epilepsy on declarative memory systems. Prog Brain Res. 2002; 135:439-53.

3. Trimble MR. Anticonvulsant drugs and cognitive function: a review of the literature. Epilepsia. 1987;28 Suppl 3:S37-45.

4. Jokeit H, Kramer G, Ebner A. Do antiepileptic drugs accelerate forgetting? Epilepsy Behav. 2005;6: 430-2.

5. Thompson PJ, Duncan JS. Cognitive decline in severe intractable epilepsy. Epilepsia. 2005;46:1780-7.

6. Aldenkamp A, Arends J. The relative influence of epileptic EEG discharges, short nonconvulsive seizures, and type of epilepsy on cognitive function. Epilepsia. 2004;45:54-63.

7. Dodrill CB. Correlates of generalized tonic-clonic seizures with intellectual, neuropsychological, emotional, and social function in patients with epilepsy. Epilepsia. 1986;27:399-411.

8. Jokeit H, Seitz RJ, Markowitsch HJ, Neumann N, Witte OW, Ebner A. Prefrontal asymmetric interictal glucose hypometabolism and cognitive impairment in patients with temporal lobe epilepsy. Brain. 1997;120:2283-94.

9. Tasch E, Cendes F, Li LM, Dubeau F, Andermann F, Arnold DL. Neuroimaging evidence of progressive neuronal loss and dysfunction in temporal lobe epilepsy. Ann Neurol. 1999;45:568-76.

10. Miller SP, Li LM, Cendes F, Tasch E, Andermann F, Dubeau F, Arnold DL. Medial temporal lobe neuronal damage in temporal and extratemporal lesional epilepsy. Neurology. 2000;54:1465-70.

11. Bernasconi A, Tasch E, Cendes F, Li LM, Arnold DL. Proton magnetic resonance spectroscopic imaging suggests progressive neuronal damage in human temporal lobe epilepsy. Prog Brain Res. 2002; 135:297-304.

12. Kotloski R, Lynch M, Lauersdorf S, Sutula T. Repeated brief seizures induce progressive hippocampal neuron loss and memory deficits. Prog Brain Res. 2002;135:95-110.

13. Trimble MR. Cognitive hazards of seizure disorders. Epilepsia. 1988;29 Suppl 1:S19-24.

14. Dodrill CB. Progressive cognitive decline in adolescents and adults with epilepsy. Prog Brain Res. 2002;135:399-407.

15. Helmstaedter C, Elger CE. The phantom of progressive dementia in epilepsy. Lancet. 1999;354:2133-4.

16. Beck H, Goussakov IV, Lie A, Helmstaedter C, Elger CE. Synaptic plasticity in the human dentate gyrus. Journal of Neuroscience. 2000;20:7080-6.

17. Sass KJ, Sass A, Westerveld M, Lencz T, Novelly RA, Kim JH, Spencer DD. Specificity in the correlation of verbal memory and hippocampal neuron loss: dissociation of memory, language, and verbal intellectual ability. J Clin Exp Neuropsychol. 1992;14:662-72.

18. Cole AJ. Is epilepsy a progressive disease? The neurobiological consequences of epilepsy. Epilepsia. 2000;41 Suppl 2:S13-22.

19. Dikmen S, Matthews CG. Effect of major motor seizure frequency upon cognitive-intellectual functions in adults. Epilepsia 1977;18:21-9.

20. Reminger SL, Kaszniak AW, Labiner DM, Littrell LD, David BT, Ryan L, Herring AM, Kaemingk KL. Bilateral hippocampal volume predicts verbal memory function in temporal lobe epilepsy. Epilepsy Behav. 2004;5:687-95.

21. Hermann B, Seidenberg M, Sears L, Hansen R, Bayless K, Rutecki P, Dow C. Cerebellar atrophy in temporal lobe epilepsy affects procedural memory. Neurology. 2004;63:2129-31.

22. Hermann B, Seidenberg M, Bell B, Rutecki P, Sheth RD, Wendt G, O'Leary D, Magnotta V. Extratemporal quantitative MR volumetrics and neuropsychological status in temporal lobe epilepsy. J Int Neuropsychol Soc. 2003;9:353-62.

23. Martin RC, Sawrie SM, Gilliam FG, Palmer CA, Faught E, Morawetz RB, Kuzniecky RI. Wisconsin Card Sorting performance in patients with temporal lobe epilepsy: clinical and neuroanatomical correlates. Epilepsia. 2000;41:1626-32.

24. Lammers MW, Hekster YA, Keyser A, Meinardi H, Renier WO, van Lier H. Monotherapy or polytherapy for epilepsy revisited: a quantitative assessment. Epilepsia. 1995;36:440-6. 
25. Aldenkamp AP, Vermeulen J, Alpherts WCJ, Overweg J, Van Parijs JAP, Verhoeff NPLG. Validity of computerized testing: Patient dysfunction and complaints versus measured changes. In: Dodson EW, Kinsbourne M, editors. Assessment of Cognitive Function in Epilepsy New York: Demos; 1991:51-68.

26. Sternberg S. High-speed scanning in human memory. Science. 1966;153:652-4.

27. Hillary FG, Chiaravalloti ND, Ricker JH, Steffener J, Bly BM, Lange G, Liu WC, Kalnin AJ, DeLuca J. An investigation of working memory rehearsal in multiple sclerosis using fMRI. Journal of Clinical and Experimental Neuropsychology. 2003;25:965-78.

28. Stroop JD. Studies of interference in serial verbal reactions. Journal of Experimental Psychology. 1935;18:643-62.

29. Marsh R, Zhu H, Schultz RT, Quackenbush G, Royal J, Skudlarski P, Peterson BS. A developmental fMRI study of self-regulatory control. Hum Brain Mapp. 2006;27:848-63.

30. Talairach J, Tournoux P. Co-planar stereotaxic atlas of the brain. 3-D Proportional System: An Approach to Cerebral Imaging. New York: Thieme Medical Publishers; 1988.

31. Seghier ML, Lazeyras F, Pegna AJ, Annoni JM, Khateb A. Group analysis and the subject factor in functional magnetic resonance imaging: Analysis of fifty right-handed healthy subjects in a semantic language task. Hum Brain Mapp. 2007 May 30.

32. Knecht S, Jansen A, Frank A, van Randenborgh J, Sommer J, Kanowski M, Heinze HJ. How atypical is atypical language dominance? Neuroimage. 2003;18:917-27.

33. Maldjian JA, Laurienti PJ, Kraft RA, Burdette JH. An automated method for neuroanatomic and cytoarchitectonic atlas-based interrogation of fMRI data sets. Neuroimage. 2003;19:1233-9.

34. O'Brien PC. Procedures for comparing samples with multiple endpoints. Biometrics. 1984;40:1079-87.

35. Läuter J. Exact $T$ and $F$ tests for analyzing studies with multiple endpoints. Biometrics. 1996;52: 964-70.

36. Hochberg Y. A sharper Bonferroni procedure for multiple tests of significance. Biometrika. 1988;75:800-2.

37. Seidenberg M, O'Leary DS, Berent S, Boll T. Changes in seizure frequency and test-retest scores on the Wechsler Adult Intelligence Scale. Epilepsia. 1981;22:75-83.

38. Seidenberg M, Geary E, Hermann B. Investigating temporal lobe contribution to confrontation naming using MRI quantitative volumetrics. J Int Neuropsychol Soc. 2005;11:358-66.

39. Represa A. Molecular mechanisms of seizure-induced cerebral plasticity. Adv Neurol. 1999;81:61-7.

40. Scharfman HE. Functional implications of seizure-induced neurogenesis. Adv Exp Med Biol. 2004;548:192-212.

41. Carpentier A, Pugh KR, Westerveld M, Studholme C, Skrinjar O, Thompson JL, Spencer DD, Constable RT. Functional MRI of language processing: dependence on input modality and temporal lobe epilepsy. Epilepsia. 2001;42:1241-54.

42. Berl MM, Balsamo LM, Xu B, Moore EN, Weinstein SL, Conry JA, Pearl PL, Sachs BC, Grandin CB, Frattali C, Ritter FJ, Sato S, Theodore WH, Gaillard WD. Seizure focus affects regional language networks assessed by fMRI. Neurology. 2005;65:1604-11.

43. Helmstaedter C, Kurthen M, Linke DB, Elger CE. Right hemisphere restitution of language and memory functions in right hemisphere language-dominant patients with left temporal lobe epilepsy. Brain. 1994;117:729-37.

44. Janszky J, Mertens M, Janszky I, Ebner A, Woermann FG. Left-sided interictal epileptic activity induces shift of language lateralization in temporal lobe epilepsy: an fMRI study. Epilepsia. 2006;47:921-7.

45. Harvey PO, Fossati P, Pochon JB, Levy R, Lebastard G, Lehericy S, Allilaire JF, Dubois B. Cognitive control and brain resources in major depression: an $\mathrm{fMRI}$ study using the n-back task. Neuroimage. 2005;26:860-9.

46. Billingsley RL, McAndrews MP, Crawley AP, Mikulis DJ. Functional MRI of phonological and semantic processing in temporal lobe epilepsy. Brain. 2001;124:1218-27.

47. Dupont S, Van de Moortele PF, Samson S, Hasboun D, Poline JB, Adam C, Lehericy S, Le Bihan D, Samson Y, Baulac M. Episodic memory in left temporal lobe epilepsy: a functional MRI study. Brain. 2000;123:1722-32.

48. Engelsen BA, Gramstad A, Thomsen T, Beneventi H, Ersland L, Smievoll Al, Lundervold A, Hugdahl K. Frontoparietal activation during delayed visuospatial recall in patients with epilepsy due to hippocampal sclerosis. Epilepsy Behav. 2006;8:565-74. 


\section{Chapter}

Hippocampal MRI volumetry at 3 Tesla: reliability and practical guidance

CRLPN Jeukens, MCG Vlooswijk, HJM Majoie, MCTFM de Krom, AP Aldenkamp, PAM Hofman, JFA Jansen, WH Backes Investigative Radiology 2009;44:509-517 


\section{Abstract}

\section{Objectives}

Although volumetry of the hippocampus is considered to be an established technique, protocols reported in literature are not described in great detail. This article provides a complete and detailed protocol for hippocampal volumetry applicable to T1-weighted magnetic resonance (MR) images acquired at 3.0 Tesla, which has become the standard for structural brain research.

\section{Materials and methods}

The protocol encompasses T1-weighted image acquisition at 3.0 Tesla, anatomic guidelines for manual hippocampus delineation, requirements of delineation software, reliability measures, and criteria to assess and ensure sufficient reliability. Moreover, the validity of the correction for total intracranial volume size was critically assessed. The protocol was applied by 2 readers to the MR images of 36 patients with cryptogenic localization-related epilepsy, 4 patients with unilateral hippocampal sclerosis, and 20 healthy control subjects.

\section{Results}

The uncorrected hippocampal volumes were $2923 \pm 500 \mathrm{~mm}^{3}$ (mean \pm SD) (left) and $3120 \pm 416 \mathrm{~mm}^{3}$ (right) for the patient group and $3185 \pm 411 \mathrm{~mm}^{3}$ (left) and $3302 \pm 411 \mathrm{~mm}^{3}$ (right) for the healthy control group. The volume of the 4 pathologic hippocampi of the patients with unilateral hippocampal sclerosis was $2980 \pm 422 \mathrm{~mm}^{3}$. The inter-reader reliability values were determined: intraclass-correlation-coefficient (ICC) $=0.87$ (left) and 0.86 (right), percentage volume difference (VD) $=7.0 \pm 4.7 \%$ (left) and $6.0 \pm 3.8 \%$ (right), and overlap ratio $(\mathrm{OR})=0.82 \pm 0.04$ (left) and $0.82 \pm 0.03$ (right). The positive Pearson correlation between hippocampal volume and total intracranial volume was found to be low: $r=0.48$ ( $p=0.03$, left) and $r=0.62$ ( $p=0.004$, right) and did not significantly reduce the volumetric variances, showing the limited benefit of the brain size correction.

\section{Conclusions}

A protocol was described to determine hippocampal volumes based on 3.0 Tesla MR images with high interreader reliability. Although the reliability of hippocampal volumetry at 3.0 Tesla was similar to the literature values obtained at 1.5 Tesla, hippocampal border definition is argued to be more confident and easier because of the improved signal-to-noise characteristics. 


\section{Introduction}

The hippocampus is an essential neuroanatomical part of the declarative long-term memory system. ${ }^{1}$ In a number of neurologic disorders, including epilepsy, ${ }^{2-4}$ Alzheimer disease, ${ }^{5,6}$ and Korsakoff syndrome, ${ }^{7}$ a relation has been proposed between hippocampal volume changes and memory-related function decline. Hippocampal volumes are usually determined on the basis of manual delineation on high-resolution 3-dimensional T1-weighted magnetic resonance (MR) image sets. Quantification of the hippocampal volume yields a more objective measure than visual inspection, potentially more sensitive to subtle changes. In this way, it becomes possible to investigate for example whether a decreased volume has prognostic value for future cognitive memory-related problems.

Unfortunately, the hippocampal volumetry protocols to delineate the hippocampus often substantially differ between studies and are not reported in great detail. ${ }^{3,8}$ Moreover, different approaches of hippocampal analysis exist (mentioned in the review article by Geuze et al. ${ }^{3}$ ). This complicates the comparison and reproducibility of measurements between different research groups. Furthermore, as 3.0 Tesla (T) magnetic resonance imaging (MRI) has become the clinical standard for neuroimaging research, the opportunity is offered to improve imaging protocols and reliability of hippocampal volume determination relative to imaging at $1.5 \mathrm{~T}$. Improved image quality at $3.0 \mathrm{~T}$ in terms of signal-to-noise, contrast-to-noise ratio, and/or spatial resolution is important for visualization of the boundaries of the hippocampal structure, which is a relatively small structure with a number of detailed subparts.

The reproducibility of hippocampal volumetry was evaluated in patients with cryptogenic localization-related epilepsy. Many of the patients with this type of chronic epilepsy display cognitive decline, especially regarding (episodic) memory function. As no structural hippocampal abnormalities are observed in these patients during regular neuroradiologic exams, it could well be that more subtle structural volume changes underlie the memory deficits. To enable the detection of such small volumetric changes of the hippocampus, a study is required that determines the lower limit of volume assessment accuracy.

The aim of this article was to provide a detailed description of all steps to be taken for the determination of the hippocampal volumes from 3-dimensional MR images acquired at 3.0 T. The protocol describes (i) the image acquisition parameters, (ii) detailed anatomic guidelines, (iii) requirements of the delineation software, and (iv) measures of and selection criteria for good reliability. Also, the validity of a standard method to correct the hippocampal volume for total brain size ${ }^{9}$ will be critically assessed. The applicability of the protocol was tested in a study population of 60 
subjects, comprising patients with epilepsy and healthy volunteers, using T1-weighted MR images.

\section{Materials and methods}

\section{Data acquisition}

Sixty subjects underwent a MRI examination on a 3.0 T whole-body MRI unit (Philips Achieva [software release 1.5.4.0]; Philips Medical Systems, Best, The Netherlands), as previously described in ${ }^{10}$. Images were acquired using a head coil with 8 channels suitable for parallel imaging. A T1-weighted 3-dimensional (3D) turbo field echo covering the entire brain was acquired with the following parameters: repetition time, 9.91 milliseconds; echo time, 4.6 milliseconds; inversion time, 3 seconds; flip angle, 8 degrees; volume matrix, $256 \times 256 \times 160$; coronal slice orientation; voxel size $1 \times 1 \times 1 \mathrm{~mm}^{3}$, and total scan time, 12 minutes.

The study population was divided in 3 groups: (i) a patient group of 36 subjects (17 women; mean age $39 \pm 12$ years, 6 left-handed, 1 ambidextrous) diagnosed with cryptogenic (nonsymptomatic) localization-related epilepsy as was determined by previous diagnostic tests (including MRI at $1.5 \mathrm{~T}$ or lower field strength), (ii) 4 patients with unilateral hippocampal sclerosis ( 3 women, mean age $57 \pm 4$ years, 1 left-handed, 1 right-sided hippocampal sclerosis), and (iii) a control group of 20 healthy subjects (11 women, mean age $40 \pm 13$ years, 2 left-handed). Both groups were matched for age and sex. Nine additional healthy volunteers (age range, 25-35 years) underwent the same MRI-examination and the corresponding images served as training data set. The results of these images were not included in the study. Approval of the local ethics committee was obtained as well as written informed consent from every subject prior to examination.

\section{Image data preparation}

Before manual delineation of the hippocampus, the images were rotated over the left-right axis to optimally position the long axis of the hippocampi perpendicular to the reformatted image plane used for delineation (mentioned in Figure 4.1). These oblique coronal slices allowed a more accurate delineation of the hippocampal crosssections. ${ }^{3,11}$ Moreover, in this way the delineation was independent on the variation of the orientation of the subject's head in the MR-scanner. Images were reformatted using customized software programmed in MATLAB (The MathWorks, Natick, Massachusetts, USA) using Sinc Interpolation to preserve image sharpness. 


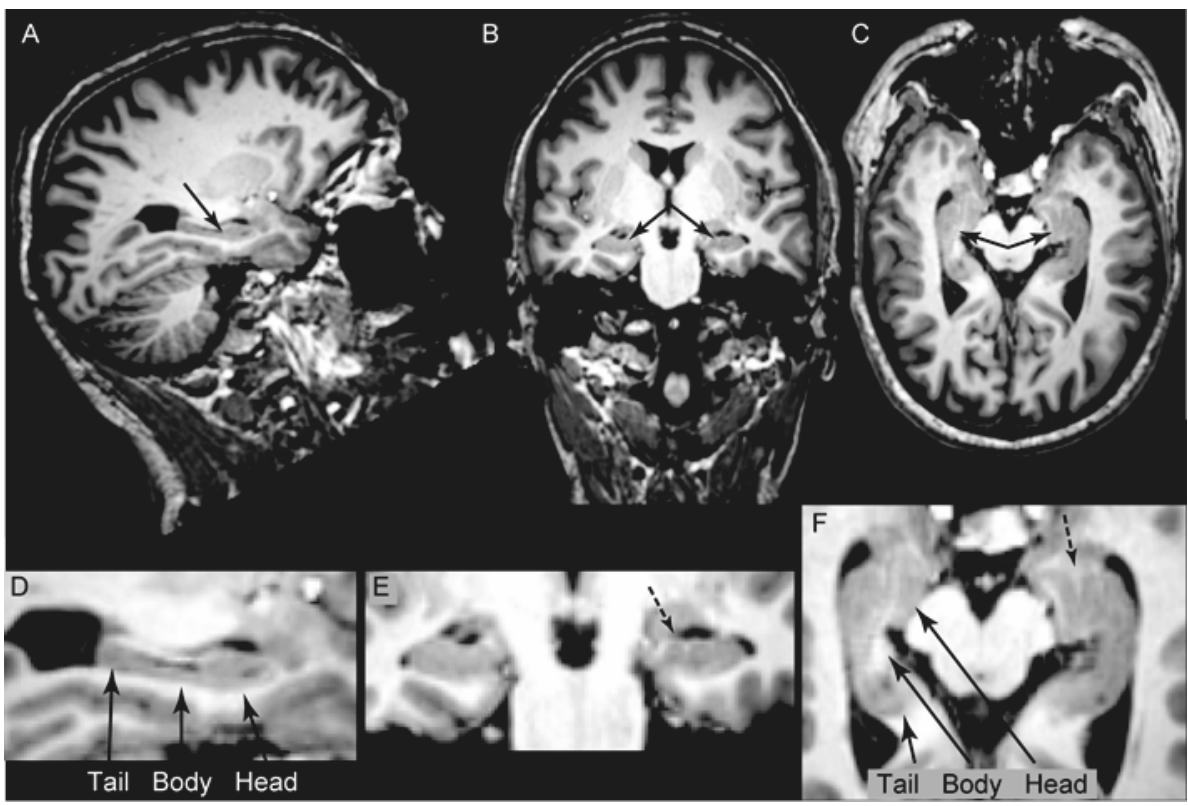

Figure 4.1 A 3D set of T1-weighted MR images acquired at 3.0 $\mathrm{T}$ demonstrating the location of the hippocampi (black arrows) in (A) sagittal, (B) coronal, and (C) transverse cross-sections. The images were rotated such that the long axis of the hippocampus was perpendicularly oriented to the oblique coronal image plane. The hippocampi are located symmetrically in the left and right hemispheres, deep in the temporal lobes. They are surrounded by the ventricles, the brain stem, parahippocampal gyrus, and the amygdala (A-C). When looking in detail at the hippocampus (D-G), the tail, body, and head part can be designated. Moreover, the digitations in the head can be recognized (dashed arrow in (E-F)). For more details on the anatomy of the hippocampus an anatomy atlas ${ }^{34}$ is referred to. The hippocampus is involved in the declarative (so-called factual) memory pathway which is part of the long term memory system. Damage to the hippocampus can result in volume loss and is associated with memory problems. ${ }^{1}$

\section{Delineation protocol}

All hippocampi were delineated by 2 image readers, who were blinded to all subject information. The application software used for the delineation was selected on a number of criteria: (i) the ability to view 3 orthogonal cross-sections simultaneously to support the delineation of difficult boundaries, (ii) the possibility to perform the delineation on a voxel-by-voxel basis, and (iii) to apply spatial smoothing. This improved the accuracy as the structure is relatively long (approximately, $4 \mathrm{~cm}$ ) and thin (typically, 9-12.5 mm in diameter) compared with the voxel size $\left(1 \times 1 \times 1 \mathrm{~mm}^{3}\right)$. In addition, it is helpful to be able to repair suboptimal delineations, by the ability to (iv) delete a single voxel of delineation, (v) temporarily hide the delineation to study the local anatomy in detail, and (vi) the ability to calculate the volume of the 
delineations. These criteria facilitate the determination of the hippocampal boundaries when strong magnifications of subregions are required. ${ }^{12}$

In this study, the freeware software program MRIcron ${ }^{12}$ was used, which fulfilled all requirements. Other software packages that have been used are nicely reviewed by Geuze et al. ${ }^{3}$

The delineation was performed in the posterior to anterior direction on the reformatted slices. After agreement between the 2 readers on the position of the most posterior slice, each second slice was delineated. The delineation protocol and the boundaries of the hippocampus are described in detail in Appendix 4.1. The anatomic guidelines were adapted from the guidelines described in articles by Jack et al. $^{9}$ and Watson et al. ${ }^{13}$ Readers agreed on the viewing settings for magnification, grayscale, smoothing, and drawing colour.

The hippocampal cross-sectional areas in the nondelineated slices were obtained by linear interpolation of the delineated slices. The hippocampal volume of every subject and every reader was calculated by multiplying the number of voxels by the voxel volume $\left(1 \mathrm{~mm}^{3}\right)$. The final volume of the hippocampus was determined as the mean of the volumes delineated by the 2 readers.

\section{Reliability measures}

Reliability measures serve to determine the inter-reader agreement for the hippocampal volumes of the 56 subjects and the intrareader agreement for the hippocampal volumes of 3 randomly chosen healthy subjects that were delineated twice by every reader.

The following reliability measures were calculated:

1. Intraclass correlation coefficient (ICC): ICC $=\mathrm{SD}_{\mathrm{bs}}{ }^{2} /\left(\mathrm{SD}_{\mathrm{bs}}{ }^{2}+\mathrm{SD}_{\mathrm{ws}}{ }^{2}\right),{ }^{14,15}$ where $\mathrm{SD}_{\mathrm{bs}}$ is the between subjects standard deviation, and $\mathrm{SD}_{\mathrm{ws}}$ the within-in subjects standard deviation. $\mathrm{SD}_{\mathrm{bs}}$ is a measure of the true biologic variation in hippocampal volumes between the subjects, whereas $\mathrm{SD}_{\mathrm{ws}}$ represents the inter-reader variation or measurement error of a hippocampal volume. The ICC quantifies the measurement error $\left(S D_{w s}\right)$ relative to the biologic variability among the subjects $\left(S_{b s}\right)$. The ICC value can range between 0 and 1 , where values closer to 1 are better. Commercial statistical software (SPSS v. 15.0 [SPSS Inc., Chicago, Illinois]) was used to calculate $\mathrm{SD}_{\mathrm{bs}}, \mathrm{SD}_{\mathrm{ws}}$, and ICC.

2. Inter-reader Volume Difference (VD): VD (\%) $=2 \times\left|V_{A}-V_{B}\right| /\left(V_{A}+V_{B}\right) \times 100 \%$, where $V_{A}$ and $V_{B}$ are the volumes delineated by reader $A$ and $B$, respectively, and $\left|V_{A}-V_{B}\right|$ is the absolute value of the volume difference. The VD expresses how strong the volumes delineated by reader $A$ and $B$ relatively differ. VD relates directly to the relative measurement variability of the delineation of an individual volume. $^{16}$ 
3. Overlap ratio $(O R)$ : The $O R$ is defined as the ratio of the overlapping volume that both readers delineated and the entire volume delineated by any reader (mentioned in Figure 4.2).

This is a measure of how much the volumes delineated by reader A and B overlap. Its value ranges from 1 (complete overlap) to 0 (no overlap). ${ }^{5,8}$

4. Bland-Altman plot. In this plot, the volume difference $\left(V_{A}-V_{B}\right)$ is displayed as a function of the average volume $\left(V_{A}+V_{B}\right) / 2$. This plot reveals whether the interreader differences depend on the (mean) volume, which could be because of a systematic error. ${ }^{17}$
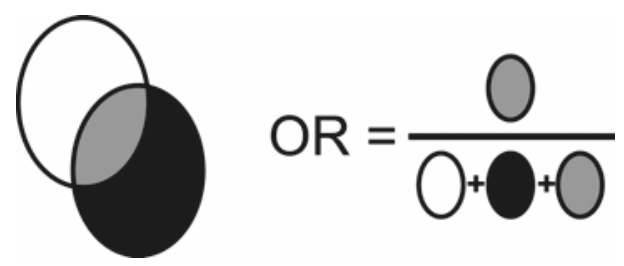

Figure 4.2 Schematic representation of the calculation of the overlap ratio (OR) of two partially overlapping regions. The OR value is defined as the overlapping region (gray) divided by the total region (sum of white, black, and gray regions).

\section{Reliability criteria}

The ICC depends both on the biologic variability of the group under study, ie, $\mathrm{SD}_{\mathrm{bs}}$, and the measurement error, ie, $\mathrm{SD}_{\mathrm{ws}}$. In literature ${ }^{14}$ it is recommended to obtain an ICC value of at least 0.90 . However, it can be expected for hippocampal volumetry that this value is too stringent, because the measurement error will have a lower bound despite all efforts of the readers. This lower bound is because of the ratio of the voxel size and the relatively small cross-sectional dimensions of the structure.

Although the hippocampus is a long structure, the short axis dimensions are relatively small. The ratio between short axis dimension and voxel size is therefore relatively small, which induces a large measurement error $\mathrm{SD}_{\mathrm{ws}}$ (mentioned in Appendix 4.2). Based on these arguments, the criterion for hippocampal volumetry was set to ICC $\geq 0.85$ in this study.

The criteria for VD and OR are taken to be VD $\leq 15 \%$ and $O R \geq 0.75$ and the criterion for the Bland-Altman plot is that there is no significant (Pearson) correlation between the volume difference and the average volume. 


\section{Correction for brain size}

In literature, it is well accepted that subjects with larger brain volumes tend to have larger brain substructures such as the hippocampus. ${ }^{9,11}$ Therefore, the hippocampal volume was corrected for intracranial volume using the covariance method described by Jack et al. ${ }^{9}$ The correction is given by the following formula (equation 1 ):

$$
V_{H C, i}(\text { corrected })=V_{H C, i}(\text { uncorrected })-B\left(I C V_{i}-\overline{I C V}\right),
$$

where $V_{H C, i}$ (corrected) is the corrected hippocampal volume of subject $i$, $V_{H C, i}$ (uncorrected) is the uncorrected hippocampal volume of subject $i, B$ is the slope of the regression of the hippocampal volume versus total intracranial volume, $I C V_{i}$ is the total intracranial volume of subject $i$, and $\overline{I C V}$ is the average intracranial volume of the subjects used in the regression. The regression analysis was performed only in the control group, to avoid any confounding effects from the potentially abnormal hippocampal volumes determined in the patients.

The total intracranial volume was determined from the MR images by automatic segmentation using routines from the Statistical Parametric Mapping software package (SPM5). ${ }^{18}$ The MR images were coregistered into the coordinate space of the standardized brain from the Montreal Neurologic Institute, from which the intracranial volume, comprising white matter, gray matter, and cerebral spinal fluid, was extracted. The resulting mask was subsequently registered on the original MR image. From this mask the intracranial volume was calculated by multiplying the number of voxels by the voxel volume. ${ }^{19}$

\section{Training of the readers}

Prior to the delineation of the study population, the readers were trained with the dataset of the 9 additional healthy volunteers under supervision of an experienced neuroradiologist. The 2 readers delineated the first 2 hippocampi jointly and thereafter 8 hippocampi separately. The delineations were compared and jointly discussed to optimize the anatomic guidelines.

\section{Delineation of the study population}

For the delineation procedure, the study population was divided in 3 groups: the first 2 groups consisted of fifteen subjects each (10 patients and 5 healthy subjects), and the third group had thirty subjects (20 patients including the 4 patients with unilateral hippocampal sclerosis and 10 healthy subjects). Within each group the patient and healthy subject data were randomly mixed and the readers were blinded for the subject's identity and pathology. The reliability measures were checked after each 5 to 10 delineated hippocampi to avoid reader drifts. 


\section{Results}

\section{Volumetry}

The uncorrected, absolute volumes of the left and right hippocampus are given in Table 4.1 for the patients and healthy controls. The left and right hippocampal volumes of the individual delineations are shown in Figure 4.3 where the horizontal axis represents the hippocampal volumes averaged over the 2 readers. Table 4.1 shows that the left hippocampus is slightly smaller than the right hippocampus; differences were $197 \mathrm{~mm}^{3}$ (6.7\%) and $117 \mathrm{~mm}^{3}$ (3.7\%) for the patient and control group, respectively. A $t$-test revealed no statistically significant differences $(p>0.05)$. The hippocampi of the patients were slightly smaller than those of the healthy controls; differences were $262 \mathrm{~mm}^{3}$ (9.0\%) and $182 \mathrm{~mm}^{3}$ (5.8\%) for the left and right hippocampus, respectively. However, a one-way analysis of variance test revealed that these differences were not statistically significant $(p>0.05)$.

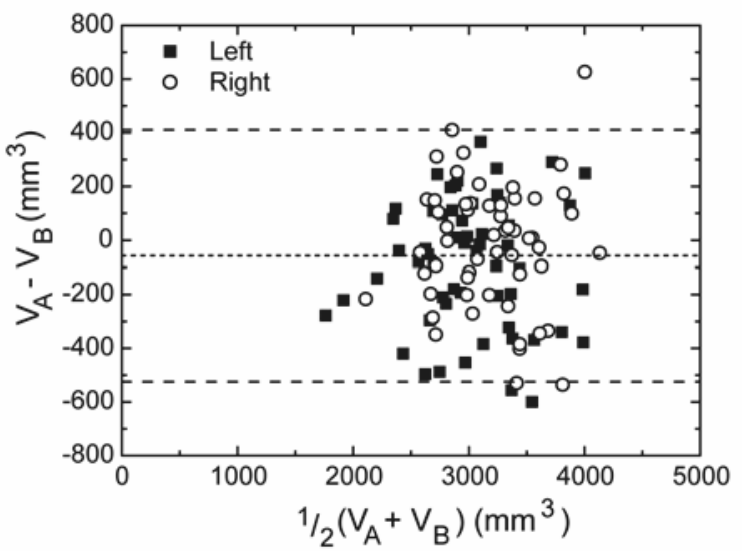

Figure 4.3 Bland-Altman plot showing the volume difference $\left(\mathrm{V}_{\mathrm{A}}-\mathrm{V}_{\mathrm{B}}\right)$ as a function of the mean volume $\left(1 / 2\left(V_{A}+V_{B}\right)\right)$ for the entire study population $(n=56)$. The dotted line represents the mean volume difference and the dashed lines represent to $95 \%$ confidence intervals.

Table 4.1 The uncorrected and brain size corrected hippocampal volumes of the epilepsy patients and healthy controls

\begin{tabular}{lccc}
\hline Hippocampal volume $\left(\mathrm{mm}^{3}\right)$ & $\mathrm{n}$ & Left & Right \\
\hline Uncorrected & 36 & $2923 \pm 500$ & \\
$\quad$ Patients & 20 & $3185 \pm 411$ & $3120 \pm 416$ \\
$\quad$ Healthy controls & & & $3302 \pm 411$ \\
$\begin{array}{l}\text { Corrected } \\
\text { Patients }\end{array}$ & 36 & $2977 \pm 418$ & $3187 \pm 351$ \\
$\quad$ Healthy controls & 20 & $3185 \pm 360$ & $3302 \pm 323$ \\
\hline
\end{tabular}

Notation: mean $\pm S D ; n$ is the number of subjects. 
Figure 4.4 shows the number of delineated voxels (i.e. volume in $\mathrm{mm}^{3}$ ) of the right and left hippocampus in the posterior to anterior direction. The number of manually delineated coronal slices was $20 \pm 2$ (mean \pm SD) for the left and $20 \pm 1$ for the right hippocampus.

The curves in Figure 4.4 also show that the left hippocampal volume was slightly smaller than the right hippocampal volume, mainly because of a smaller tail and head. The mean difference in number of voxels between the delineations of the 2 readers demonstrates that the tail and head represent the regions where the inter-reader variability is largest.

The average time needed to delineate one hippocampus was $33 \pm 9$ minutes (range, 19-68). The time investment for the volumetry of a study population containing 56 subjects is approximately 56 subjects $\times 2$ hippocampi $\times 33$ minutes $\times 2$ readers $=123$ hours. Prior to this, every reader had spent about 25 hours for training and discussion to reach consensus on the delineation protocol. In addition, the data preparation and data analysis took approximately 40 hours by a physicist who had experience in MRI and MATLAB programming.

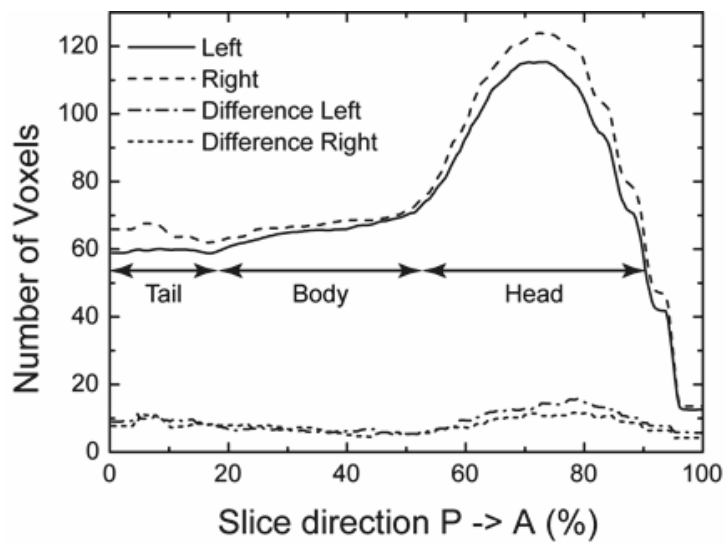

Figure 4.4 Number of delineated voxels for the left (solid curve) and right (dashed curve) hippocampus averaged over 56 delineations traversing in the posterior $(P, 0 \%)$ to anterior $(A, 100 \%)$ direction. The modulation of the curves revealed the shape of the hippocampus: the tail, body, and head subparts. The dotted (left) and dashed dotted line (right) show the mean difference between the delineations of reader $A$ and $B$.

\section{Reliability measures}

Table 4.2 shows the values of the inter-reader and intrareader reliability measures calculated for the left and right hippocampal volumes. No difference was observed between the reliability measures of the left and right hippocampal volumes separately ( $n=56$ each) as compared with all volumes $(n=112)$. The $S_{\text {ws }}$ was found to be 2 to 3 
times smaller than the $\mathrm{SD}_{\mathrm{bs}}$, which is expressed in the high ICC values. OR and VD were calculated for each pair of delineations and the mean and SD are also listed in Table 4.2. All reliability measures met the criteria defined except for the ICC value of the intrareader A. Figure 4.3 shows the Bland-Altman plot of all delineations made by the 2 readers. There was no significant correlation $(r=0.07, p=0.59$ [left] and $r=-0.017$, $p=0.9$ [right]) between the volume difference and mean volume.

Of 112 delineations, 10 (i.e. $8.9 \%$ ) did not meet the defined standards and were jointly reexamined by the 2 readers to reach consensus. The consensus value was within $6 \%$ of the average of the 2 volumes as determined by the readers separately.

\section{Volumetry in patients with hippocampal sclerosis}

The volume of the 4 pathologic hippocampi was $2980 \pm 400 \mathrm{~mm}^{3}$. The ICC was 0.70 , the OR was $0.78 \pm 0.07$, and the VD was $12.3 \pm 8.8 \%$ (mentioned in Table 4.2).

Table 4.2 Inter- and intra-reader variabilities and reliability measures for the left $(L)$ and right (R) hippocampus

\begin{tabular}{lccccccc}
\hline & $\mathrm{n}$ & Side & $\mathrm{SD}_{\mathrm{bs}}$ & $\mathrm{SD}_{\text {ws }}$ & ICC & OR & VD (\%) \\
\hline Inter-reader & 112 & $\mathrm{~L} \& \mathrm{R}$ & 445 & 175 & 0.87 & $0.82 \pm 0.03$ & $6.5 \pm 4.3$ \\
& 56 & $\mathrm{~L}$ & 466 & 178 & 0.87 & $0.82 \pm 0.04$ & $7.0 \pm 4.7$ \\
& 56 & $\mathrm{R}$ & 404 & 162 & 0.86 & $0.82 \pm 0.03$ & $6.0 \pm 3.8$ \\
Intra-reader A & 6 & $\mathrm{~L} \& \mathrm{R}$ & 214 & 124 & 0.59 & $0.87 \pm 0.01$ & $4.3 \pm 3.1$ \\
Intra-reader B & 6 & $\mathrm{~L} \& \mathrm{R}$ & 171 & 63 & 0.88 & $0.91 \pm 0.01$ & $1.8 \pm 0.8$ \\
\hline
\end{tabular}

The inter-reader reliability measures of the 4 pathological hippocampi of the patients with hippocampal sclerosis (HS) are given separately. Notation: mean $\pm S D ; n$ is the number of subjects.

\section{Correction for brain size}

Figure 4.5 shows the volume of the left and right hippocampus as a function of total intracranial volume for the healthy control group with a linear regression of the data. For the left hippocampus, the slope was $B=0.0016(r=0.48, p=0.03)$ and for the left hippocampus $B=0.0020(r=0.62, p=0.004)$. The data points show a relatively large spread which is reflected by the low $r$-value. The mean total intracranial volume of the healthy controls and volunteers was $(1.28 \pm 0.12) \times 10^{6} \mathrm{~mm}^{3}$. The individual intracranial volumes were used to correct hippocampal volumes according to Equation 1. The corrected volumes are listed in Table 4.1. The variances of the corrected volumes are not significantly decreased relative to the uncorrected volume, which was determined using an F-test. 


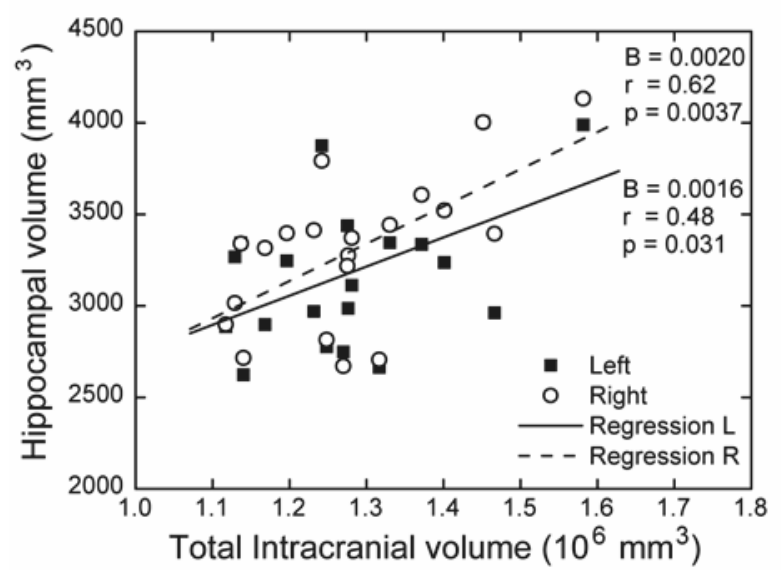

Figure 4.5 Left and right hippocampal volume as a function of the total intracranial volume for the healthy controls $(n=20)$.

\section{Discussion}

In this study, a protocol was described in detail to determine the hippocampal volumes from T1-weighted MR images acquired at 3.0 T. The protocol included the image acquisition parameters, anatomic guidelines, requirements of the delineation software, reliability measures and their criteria, and an analysis of correction for brain size. The protocol was performed on a study population of 36 patients with cryptogenic (nonsymptomatic) epilepsy, 4 patients with unilateral hippocampal sclerosis, and 20 healthy volunteers. The results show that the volumes of hippocampi, without neuroradiologic abnormalities, can be reliably determined with typical inter-reader variations less than $7 \%$, which is close to the theoretical limit of $6 \%$ to $8 \%$ (mentioned in appendix 4.2 ), and an inter-reader overlap ratio of 0.82 . As, for example, in epilepsy patients with hippocampal sclerosis up to $30 \%$ hippocampal volume loss $^{20}$ have been reported, MR volumetry measurements provide sufficient reliability.

\section{Acquisition and Delineation protocol}

In this study, coronal images were acquired with an isotropic voxel size of $1 \mathrm{~mm}$. To determine structural hippocampal abnormalities in the neuroradiologic clinic, usually oblique coronal images are acquired which are orientated perpendicular to the long axis of the hippocampi. However, such an oblique image acquisition requires longer acquisition times than straightforward coronal images. On the other hand, transformation of coronal images to the orientation perpendicular to the long axis 
may involve (linear) interpolations that spatially smoothes the images and therefore degrade the quality of the originally acquired images. To minimize these partial volume effects and to preserve the definitions of the hippocampal borders Sinc Interpolation was used which preserves the high spatial-frequency contents (image sharpness) of the images.

In literature, the anatomic guidelines show a great variety in anatomic boundaries, for example, whether the alveus, subiculum, fimbriae, or uncus are included. ${ }^{3,4,8,11}$ This leads to a significant variation in reported hippocampal volumes (mentioned in Table 4.3) and complicates the comparison between different studies. Within one study, the boundaries can be defined in a more or less arbitrary way, as long as different readers delineate consistently. However, the transition of the hippocampus to the amygdala is an example of a hippocampal boundary that is particularly hard to discern. In such cases, the anatomic guidelines provided in this study give a practical rule supporting reproducible delineation between different readers.

Table 4.3 Hippocampal volumes reported in literature

\begin{tabular}{|c|c|c|c|c|}
\hline \multirow[t]{2}{*}{ Reference } & \multicolumn{2}{|c|}{ Hippocampal volume $\left(\mathrm{mm}^{3}\right)$} & \multirow{2}{*}{$\begin{array}{l}\text { Brain size } \\
\text { corrected }\end{array}$} & \multirow[t]{2}{*}{ Study population } \\
\hline & Left & Right & & \\
\hline Bigler et al. $^{33}$ & 2538 & 2599 & yes & Healthy, $n=96$ \\
\hline Free et al. ${ }^{26}$ & 2772 & 2799 & yes & Healthy, $n=32$ \\
\hline Hammers et al. ${ }^{22}$ & 2317 & 2525 & yes & Healthy, $n=30$ \\
\hline Jack et al. ${ }^{9}$ & 2500 & 2800 & yes & Healthy, $n=52$ \\
\hline Mackay et al.) & 2460 & 2590 & no & Healthy, $n=20$ \\
\hline Pantel et al. ${ }^{8}$ & 1975 & 1987 & no & $\begin{array}{l}\text { Healthy, } n=8 \\
\text { Patients }{ }^{a}, n=7\end{array}$ \\
\hline Van Paesschen et al. ${ }^{20}$ & 5405 & 5631 & yes & Healthy, $n=22$ \\
\hline \multirow[t]{2}{*}{ Soininen et al. ${ }^{6}$} & 3353 & 3714 & yes & Healthy, $n=16$ \\
\hline & 3441 & 3550 & yes & Patients $^{b} n=16$ \\
\hline Tisserand et al. $^{28}$ & 1427 & 1501 & yes & Healthy, $n=61$ \\
\hline Watson et al. ${ }^{13}$ & 4903 & 5264 & no & Healthy, $n=15$ \\
\hline \multirow[t]{2}{*}{ Zipursky et al. ${ }^{25}$} & 1990 & 2070 & yes & Healthy, $n=20$ \\
\hline & 2010 & 2040 & yes & Patients $^{\mathrm{a}}, \mathrm{n}=22$ \\
\hline
\end{tabular}

These studies were all performed on 1.5 Tesla MRI systems using oblique coronal slices.

${ }^{a}$ Patients suffering from schizophrenia, ${ }^{b}$ patients suffering from age-associated memory impairment.

\section{Magnetic field strength}

At higher magnetic field strength, the signal-to-noise ratio and/or the spatial resolution may improve, which in theory leads to improved white matter to gray matter border definitions and delineations. Two studies have shown that the higher magnetic field strength does not influence the value of the measured hippocampal volumes, meaning that volumes determined at 3.0 T can be compared with those determined at $1.5 \mathrm{~T}^{16,21}$ Briellmann et al. demonstrated with almost identical pulse 
sequences in 8 healthy subjects that the measurement error was slightly lower at 3.0 $\mathrm{T}(3.4 \pm 2.5 \%)$ as compared with $1.5 \mathrm{~T}(4.0 \pm 3.1 \%)$. However, the main source of the measurement variability was the manual hippocampal volume measurement, which was higher $(6.0 \pm 3.9 \%)$ than the measurement error at both field strengths. Therefore, they concluded that there is no major advantage in using 3.0 $\mathrm{T}$ as compared with $1.5 \mathrm{~T}$ for hippocampal volumetry, because the anatomic images are already of very high standard at $1.5 \mathrm{~T}^{16}$ and that manual delineation errors are the largest source of uncertainty.

T1-weighted MRI at 3.0 T has the drawback of longer acquisition times compared with 1.5 T. Two reasons for the increased acquisition time at 3.0 $\mathrm{T}$ are the facts that (i) the $\mathrm{T} 1$ relaxation times of cerebral tissue are longer and (ii) that the difference in T1 relaxation time between gray and white matter is smaller at 3.0 T relative to $1.5 \mathrm{~T}$. The latter prompts the need for the use of an inversion pulse to improve the gray/white matter contrast at 3.0 T, which lengthens the scan time. In our neuroradiologic clinic, all T1-weighted protocols at 3.0 $\mathrm{T}$ take more time compared with comparative protocols at $1.5 \mathrm{~T}$ but display improved signal-to-noise characteristics, which provides more confidence and easier hippocampal structure evaluation. Both patients with epilepsy and healthy volunteers displayed strong compliance to the 12-minutes scanning protocol in the current study. No severe motion artifacts were observed in any of the participants. We did not use accelerating factors for the parallel imaging coil to speed up the image acquisition. Scan time may be strongly reduced by using parallel imaging with 8,16 , or 32 head coil elements for less compliant patients. By using parallel imaging with multiple small coil elements, the signal-to-noise ratio per unit image acquisition time strongly improves. Thomas et al. ${ }^{22}$ recently exploited a 7.0 T MRI system equipped with a 16-channel head coil for hippocampal imaging (acquisition time, 9 minutes) in healthy volunteers and achieved $700 \mu \mathrm{m}$ isotropic voxels to demonstrate in vivo depiction of anatomic clarity of the hippocampal formation's subcomponents. However, at such a high field many technical limitations arise, including in-plane dephasing and loss of signal at tissue-air interfaces and near the skull base. Furthermore, radiofrequency power deposition variations were reported across the brain, which may lead to miscalibration of the desired flip angle and degradation of the tissue contrast.

\section{Automated delineation}

To avoid inter-reader variability of the hippocampal structures and to enable large population studies, current developments of automated delineation software are of importance. Custom software remains popular and attractive in neuroradiologic research. However, no empirical evidence exists indicating that this leads to significant differences. ${ }^{3}$ A recent study by Colliot et al. ${ }^{23}$ showed that automated delineation software is able to detect significant volume differences in both patients with 
Alzheimer disease and patients with mild cognitive impairment and to provide results that are in agreement with a number of studies using manual hippocampus delineation. Interestingly, the study by Giesel et al. ${ }^{24}$ demonstrated that differences between controls and patients with Alzheimer disease strongly correlated for a manual direct hippocampal volume measurement and an automated indirect measurement of the temporal horn volume, which was 10 times faster. Two previous studies have compared automated delineation with manual delineation and showed that the results of the automated procedure were as accurate as the manual procedure. $^{5,25}$ To compare the automated with the manual procedure, Carmichael et al. reported $\mathrm{OR}$ values around $0.6^{5}$ and Hammers et al. reported similarity index values in the range of 0.76 to $0.83,{ }^{25}$ which corresponds to OR values of 0.61 to 0.71 . These values were lower than the OR values achievable with manual delineation reported in this study (Table 4.2). Two other studies that compared manual and automated delineation showed that there was a poor correlation between the hippocampal volumes determined using manual and automated delineation ${ }^{23,26}$ and that the automatically determined hippocampal volumes considerably deviate up to $35 \%$ from the manually determined volumes. ${ }^{27}$ It can be concluded that currently available automated delineation procedures need further improvement and that manual delineation remains the gold standard. ${ }^{5,25,27}$

\section{Hippocampal volumes}

The hippocampal volumes that were determined in this study were in agreement with values found in literature (compare Table 4.1 with Table 4.3). Hippocampal laterality differences and differences between patients and healthy controls were not statistically significant. However, the literature values reported in Table 4.3 reflect the same trend that the left hippocampus is slightly smaller than the right hippocampus. The trend that patients exhibit smaller hippocampal volumes is not reflected by values found in literature (Table 4.3). ${ }^{6,28}$ However, to confidently infer on volumetric differences between the left and right hippocampus and between patients and healthy controls much larger populations have to be studied, which is beyond the scope of this study. The current study provides data that may serve as input to such sample size estimations.

\section{Reliability of the delineated volumes}

The ICC is a measure that can be thought of as the fraction of the total variance that is attributed to true biologic variation rather than measurement error. ${ }^{14}$ It is recommended that this fraction should be larger than 0.90 , so that the measurement error is small when compared with the biologic variation. ${ }^{14}$ However, the same measurement error can lead to a low ICC when the biologic variance in a group is small. This is illustrated by the rather low ICC value found in the reproducibility measurements of intrareader A (Table 4.2), where the biologic variation was low 
among the healthy controls. However, from the OR and VD values it can be concluded that the reproducibility was high. This demonstrates that inferences on the reliability solely based on ICC are inadequate to determine the reliability of the delineations.

ICC values reported in literature vary from 0.73 up to $0.95 .^{2,4,6,8,13}$ Our results show that an inter-reader ICC higher than 0.85 can be achieved. The ICC and VD only consider the size of the volumes and ignore whether the volumes encompass the same voxels. Theoretically, 2 readers can delineate an equal volume in a completely different, nonoverlapping region. This would not be revealed by the ICC or the VD. Therefore, it is important to determine the OR as well. Only a few studies reported OR values in the range of 0.45 to $0.82 .{ }^{5,8,25}$ Our results show that an overlap ratio higher than 0.80 is feasible. Values for the mean VD reported in literature were in the range of 3.4\% to $9 \%{ }^{16,25,29}$ The average VD values found in Table 4.2 agree with these values.

\section{Applicability for abnormal hippocampi}

Four patients with hippocampal sclerosis were included in the study to demonstrate the applicability of the protocol for structurally abnormal hippocampi. The observed reliability measures ICC, OR, and VD were slightly worse but still acceptable compared with the other hippocampi. An explanation for this decrease in reliability might be the loss of structure and locally deviating signal levels, which result in interpretation differences to delineate he hippocampal borders between the 2 observers.

\section{Correction for brain size}

Several studies have advised to correct the hippocampal volumes for brain size, as larger brains on average have larger brain structures. ${ }^{11,29,30}$ In this study, the covariance method proposed by Jack et al. ${ }^{9}$ was followed, but other methods are described which all use the relation between total intracranial volume and hippocampal volume. Although the brain size correction introduces an additional source of error because of the uncertainty in the regression, the study by Mathalon et al. showed that volumes corrected for might give better correlations with age and diagnostic status. ${ }^{30}$

Based on the results of this study, the usefulness of the correction can be questioned for the following reasons. The (linear) relation between the total intracranial volume size and the hippocampal volume is weak (Figure 4.5). To ensure that this was not because of errors in the automated total intracranial volume determination, 5 brain masks were visually checked in detail. Also an independent dataset consisting of 61 healthy subjects revealed a weak correlation $(r=0.42, p=0.0008)$ (Figure 4.6). These data were extracted from an article published by Tisserand et al. ${ }^{31}$ Moreover, the Bvalues found in Figure 4.5 and 5.6 differ by a factor of 2 indicating the difficulty in determining the relation accurately. Two other studies also reported a weak 
correlation. $^{32,33}$ Although a relation between total intracranial volume and hippocampal volume seems to exist, the $r$-value is low and a large population is required to reliably determine the correlation. Finally, application of the brain size correction did not significantly reduce the variances of the hippocampal volumes.

In the review study of Geuze et al., ${ }^{3}$ it is reported that in $34 \%$ of the studies no brain size correction is applied. Whether this is because of the above reasons or negligence is not clear. We conclude that correction of the hippocampal volumes for total intracranial volume is not straightforward and that the correlation between the hippocampal volume and total intracranial volume should be critically assessed before using it for correction.

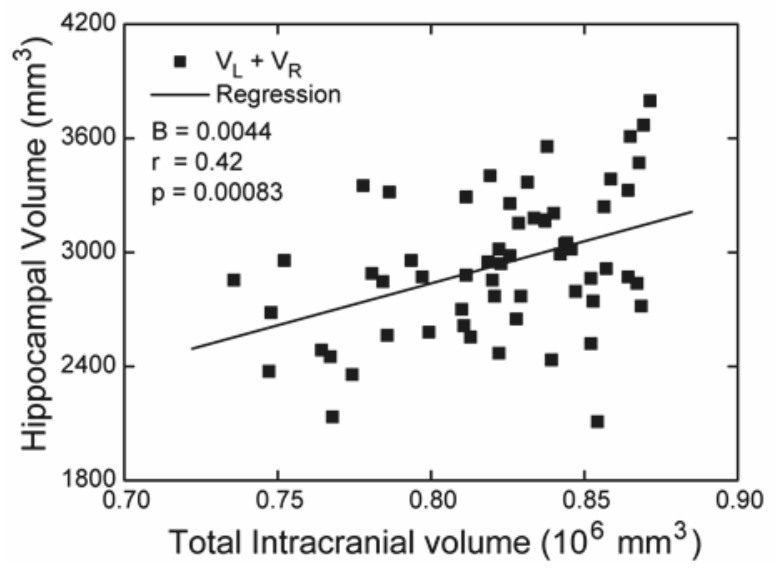

Figure 4.6 Total hippocampal volume as a function of total intracranial volume for 61 healthy subjects. The data are obtained from a paper published by Tisserand et al. ${ }^{31}$

\section{Volumetric versus visual assessment}

Hippocampal atrophy can be visually detected by experienced readers without timeconsuming volumetry. However, we feel that a quantitative assessment of the hippocampal volumetry is more objective when strict guidelines for hippocampal border definitions are complied with. Moreover, volumetry is most likely more sensitive to subtle changes than visual assessment especially for the analysis of correlations to other (eg, neuropsychologic) measures.

\section{Conclusions}

This study described in detail a protocol for the determination of hippocampal volumes with high reliability based on 3.0 T MR images. Image acquisition parameters, anatomic guidelines, requirements of the manual delineation software, reliability 
measures, and criteria to ensure a good reliability were given. The applicability of the protocol was demonstrated in a relatively large study population of epilepsy patients and healthy controls. Although there is currently no clinical indication to perform hippocampal volumetry, this technique can be used to provide supportive independent information on the hippocampus in patients with memory dysfunction. 


\section{Appendix 4.1}

\section{Anatomic guidelines- boundaries of the hippocampus}

The anatomic guidelines are adapted from the guidelines described in articles by Jack et al. ${ }^{9}$ and Watson et al. ${ }^{13}$ After familiarizing oneself with the rough shape of the hippocampus by scrolling back and forth through the (oblique) coronal slices, the hippocampus is delineated on these images according to the boundaries described in this appendix. The hippocampus is delineated on each second slice in the posterior to anterior direction. For detailed images of the boundaries, we refer to an anatomy atlas. $^{34}$

\section{Posterior boundary}

- Moving from the posterior to anterior direction, the first coronal slice is where the crux of the fornix is clearly visible. This structure should be well-defined with a clear dark-bright separation.

\section{Lateral boundary}

- $\quad$ Fluid of the temporal horn of the ventricle.

- White matter bordering the gray matter of the hippocampus. All gray matter should be included, inclusion of voxels brighter than the gray matter voxels, ie, representing small amounts of white matter is acceptable. However, when a voxel is very bright it should be excluded.

\section{Inferior boundary}

- White matter of the parahippocampal gyrus.

\section{Medial boundary}

- In the tail: take care not to include the isthmus (the small posterior part of the parahippocampal gyrus). The boundary between the hippocampus and the isthmus is defined by the groove: the most lateral point of the groove is taken and from there straight down (to inferior). The voxels that are gray but not really white yet are to be included.

- In the coronal slice where the pulvinar becomes visible (it helps to locate the posterior boundary on the sagittal slices), the isthmus turns into the subiculum which is to be included. The subiculum is included until the most medial point in the turn downwards, if it is clear that it is no fluid or white matter. The connection between the hippocampus and the subiculum can be very thin.

- $\quad$ Anterior, in the head: at some point there is no longer a subiculum that continues in the parahippocampal gyrus. When the amygdala becomes visible in the coronal slices, the 
subiculum ends. Furthermore, be aware that the hippocampus not necessarily reaches the fluid. Take care that in this region not too much is included.

\section{Superior boundary}

- In the tail: medial superiorly the boundary is the white matter (not included), except when the gray matter is clearly a different structure. Note that the tail of the hippocampus stretches a bit upwards at its end, this should be included.

- The alveus should not be included in the most posterior slices where it is part of the fornix and still runs diagonally. More anteriorly, in the body of the hippocampus, the alveus lies horizontally on top of the hippocampus and should be included. The fornix can be found quite far anteriorly, and it looks like a layer of white voxels. Only the first row of white voxels is included.

- In the head: the alveus evolves into the fimbriae looking like a white structure or line, and is included. Sometimes this line is a few voxels thick, in that case only the first row of white voxels is included. When there is a space between the alveus and the hippocampus, this should not be included. When there is gray matter above the alveus, this is most likely not hippocampus and should not be included. Because the hippocampus is a curled structure, there can be a white line visible within the hippocampus. On the sagittal slices, the outer white line can usually clearly be observed.

- Note that the white line of the fimbriae may become fuzzy in the head, take care to include the white line, but to exclude the gray matter of the amygdala.

- The boundary between the hippocampus and the amygdala is taken to be a virtual line at the location where the amygdala sprouts from the hippocampus.

\section{General}

- Do make use of the sagittal and transverse images as some boundaries are more clearly visualized.

- $\quad$ Additional guidelines for fluid boundaries: do not include the black or dark gray voxels, for white matter boundaries: do include all the gray voxels.

- Sometimes, the hippocampus includes cysts (fluid filled pockets). When a cyst has the size of 2 really black (ie, fluid) voxels as well as a number of reasonably black ones, the cyst should be excluded. The small cysts, ie, a size of less than 4 voxels may be excluded.

- $\quad$ Finally, when the delineation on the coronal slices is finished, the delineation is screened on the sagittal and transverse slices for irregularities and outliers. 


\section{Appendix 4.2: Volume error analysis}

Here, the theoretical lower limits are derived for the accuracy of hippocampal volume determination by manual delineation procedures. To this end, the hippocampus is modelled by a relatively long cylindrical volume with varying cross-sectional dimensions. It assumed that the length is $L=40 \mathrm{~mm}$ and the cross-sectional circular diameters typically vary between $d 9$ to $12.5 \mathrm{~mm}$. The diameter values were derived from the measured cross-sectional areas $A=\frac{\pi}{4} d^{2}=60-120 \mathrm{~mm}^{2}$ in Figure 4.3. Note that approximately 40 to $50 \%$ of the hippocampus corresponds to the small diameter, while only 10 to $15 \%$ corresponds to the large diameter.

Quantitative error analysis states that independent errors in the cylinder dimensions can be relatively added according to ${ }^{35}$ :

$$
\left(\frac{\Delta V}{V}\right)^{2}=2\left(\frac{\Delta d}{d}\right)^{2}+\left(\frac{\Delta L}{L}\right)^{2}
$$

where $V=\frac{\pi}{4} d^{2} L$ is the volume of the cylinder. The error formula expresses to what extent errors in diameter ( $\Delta d)$ and length ( $\Delta L$ ) contribute to the calculation of the relative volume error $(\Delta V / V)$. It can be seen that errors in the diameters contribute much stronger to the volume error than errors in the length, for 2 reasons: (i) the factor 2 , related to the 2 cross-sectional dimensions and (ii) because of the relatively small diameter (and large length) values in the denominator of the first (second) term. Table 4.4 lists the results of the error analysis for 2 different error values in diameter and length.

Table 4.4

\begin{tabular}{ccccc}
\hline$\Delta d(\mathrm{~mm})$ & $\Delta L(\mathrm{~mm})$ & $\Delta d / d(\%)$ & $\Delta L / L(\%)$ & $\Delta V / V(\%)$ \\
\hline 1 & 1 & $8-11$ & 2.5 & $12-16$ \\
0.5 & 0.5 & $4-5.5$ & 1.2 & $6-8$ \\
\hline
\end{tabular}

In our opinion, is the assumption of 1 voxel (size, $1 \times 1 \times 1 \mathrm{~mm}^{3}$ ) error too conservative, because it considers that over the entire perimeter one voxel is added. Assuming a circumferentially averaged error of a half pixel (ie, $0.5 \mathrm{~mm}$ ) is more realistic. This analysis shows that $6 \%$ to $8 \%$ is the theoretical lower boundary for the error in the volume in manual hippocampal volume delineation for voxel sizes of $1 \times 1$ $x 1 \mathrm{~mm}^{3}$. 


\section{References}

1. Marieb EN. Human anatomy \& Physiology. San Francisco, California: Pearson Education Inc.; 2004.

2. Araujo D, Santos AC, Velasco TR, Wichert-Ana L, Terra-Bustamante VC, Alexandre V, Jr., Carlotti CG, Jr., Assirati JA, Jr., Machado HR, Walz R, Leite JP, Sakamoto AC. Volumetric evidence of bilateral damage in unilateral mesial temporal lobe epilepsy. Epilepsia. 2006;47:1354-9.

3. Geuze E, Vermetten E, Bremner JD. MR-based in vivo hippocampal volumetrics: 1. Review of methodologies currently employed. Molecular psychiatry. 2005;10:147-59.

4. Mackay CE, Webb JA, Eldridge PR, Chadwick DW, Whitehouse GH, Roberts N. Quantitative magnetic resonance imaging in consecutive patients evaluated for surgical treatment of temporal lobe epilepsy. Magnetic resonance imaging. 2000;18:1187-99.

5. Carmichael OT, Aizenstein HA, Davis SW, Becker JT, Thompson PM, Meltzer CC, Liu Y. Atlas-based hippocampus segmentation in Alzheimer's disease and mild cognitive impairment. Neuroimage. 2005; 27:979-90.

6. Soininen HS, Partanen K, Pitkanen A, Vainio P, Hanninen T, Hallikainen M, Koivisto K, Riekkinen PJ, Sr. Volumetric MRI analysis of the amygdala and the hippocampus in subjects with age-associated memory impairment: correlation to visual and verbal memory. Neurology. 1994;44:1660-8.

7. Visser PJ, Krabbendam L, Verhey FR, Hofman PA, Verhoeven WM, Tuinier S, Wester A, Den Berg YW, Goessens LF, Werf YD, Jolles J. Brain correlates of memory dysfunction in alcoholic Korsakoff's syndrome. J Neurol Neurosurg Psychiatry. 1999;67:774-8.

8. Pantel J, O'Leary DS, Cretsinger K, Bockholt HJ, Keefe H, Magnotta VA, Andreasen NC. A new method for the in vivo volumetric measurement of the human hippocampus with high neuroanatomical accuracy. Hippocampus. 2000;10:752-8.

9. Jack CR, Jr., Twomey CK, Zinsmeister AR, Sharbrough FW, Petersen RC, Cascino GD. Anterior temporal lobes and hippocampal formations: normative volumetric measurements from MR images in young adults. Radiology. 1989;172:549-54.

10. Jansen JF, Vlooswijk MC, Majoie HM, de Krom MC, Aldenkamp AP, Hofman PA, Backes WH. White matter lesions in patients with localization-related epilepsy. Invest Radiol. 2008;43:552-8.

11. Jack CR Jr, Theodore WH, Cook M, McCarthy G. MRI-based hippocampal volumetrics: data acquisition, normal ranges, and optimal protocol. Magnetic resonance imaging. 1995;13:1057-64.

12. http://www.sph.sc.edu/comd/rorden/mricron/.

13. Watson C, Andermann F, Gloor P, Jones-Gotman M, Peters T, Evans A, Olivier A, Melanson D, Leroux G. Anatomic basis of amygdaloid and hippocampal volume measurement by magnetic resonance imaging. Neurology. 1992;42:1743-50.

14. Tofts P. Quantitative MRI of the brain, measuring changes caused by disease. Chichester, West Sussex; Hoboken, New Jersey: John Wiley \& Sons Ltd; 2003.

15. Jansen JF, Kooi ME, Kessels AG, Nicolay K, Backes WH. Reproducibility of quantitative cerebral T2 relaxometry, diffusion tensor imaging, and $1 \mathrm{H}$ magnetic resonance spectroscopy at 3.0 Tesla. Invest Radiol. 2007;42:327-37.

16. Briellmann RS, Syngeniotis A, Jackson GD. Comparison of hippocampal volumetry at 1.5 Tesla and at 3.0 Tesla. Epilepsia. 2001;42:1021-4.

17. Bland JM, Altman DG. Statistical methods for assessing agreement between two methods of clinical measurement. Lancet. 1986;1:307-10.

18. Wellcome Department of Cognitive Neurology. http://www.fil.ion.ucl.ac.uk/spm/.

19. Luders E, Steinmetz $H$, Jancke L. Brain size and grey matter volume in the healthy human brain. Neuroreport. 2002;13:2371-4.

20. Van Paesschen W, Connelly A, King MD, Jackson GD, Duncan JS. The spectrum of hippocampal sclerosis: a quantitative magnetic resonance imaging study. Ann Neurol. 1997;41:41-51.

21. Scorzin JE, Kaaden S, Quesada CM, Muller CA, Fimmers R, Urbach H, Schramm J. Volume determination of amygdala and hippocampus at 1.5 and 3.0T MRI in temporal lobe epilepsy. Epilepsy Res. 2008;82:29-37.

22. Thomas BP, Welch EB, Niederhauser BD, Whetsell WO, Jr., Anderson AW, Gore JC, Avison MJ, Creasy JL. High-resolution 7T MRI of the human hippocampus in vivo. J Magn Reson Imaging. 2008;28: 1266-72. 
23. Colliot O, Chetelat G, Chupin M, Desgranges B, Magnin B, Benali H, Dubois B, Garnero L, Eustache F, Lehericy S. Discrimination between Alzheimer disease, mild cognitive impairment, and normal aging by using automated segmentation of the hippocampus. Radiology. 2008;248:194-201.

24. Giesel FL, Thomann PA, Hahn HK, Politi M, Stieltjes B, Weber MA, Pantel J, Wilkinson ID, Griffiths PD, Schroder J, Essig M. Comparison of manual direct and automated indirect measurement of hippocampus using magnetic resonance imaging. Eur J Radiol. 2008;66:268-73.

25. Hammers A, Heckemann R, Koepp MJ, Duncan JS, Hajnal JV, Rueckert D, Aljabar P. Automatic detection and quantification of hippocampal atrophy on MRI in temporal lobe epilepsy: a proof-ofprinciple study. Neuroimage. 2007;36:38-47.

26. Mueller CA, Scorzin J, Koenig R, Urbach H, Fimmers R, Zentner J, Lehmann TN, Schramm J. Comparison of manual tracing versus a semiautomatic radial measurement method in temporal lobe MRI volumetry for pharmacoresistant epilepsy. Neuroradiology. 2007;49:189-201.

27. Tae WS, Kim SS, Lee KU, Nam EC, Kim KW. Validation of hippocampal volumes measured using a manual method and two automated methods (FreeSurfer and IBASPM) in chronic major depressive disorder. Neuroradiology. 2008;50:569-81.

28. Zipursky RB, Marsh L, Lim KO, DeMent S, Shear PK, Sullivan EV, Murphy GM, Csernansky JG, Pfefferbaum A. Volumetric MRI assessment of temporal lobe structures in schizophrenia. Biol Psychiatry. 1994;35:501-16.

29. Free SL, Bergin PS, Fish DR, Cook MJ, Shorvon SD, Stevens JM. Methods for normalization of hippocampal volumes measured with MR. AJNR Am J Neuroradiol. 1995;16:637-43.

30. Mathalon DH, Sullivan EV, Rawles JM, Pfefferbaum A. Correction for head size in brain-imaging measurements. Psychiatry Res. 1993;50:121-39.

31. Tisserand DJ, Visser PJ, van Boxtel MP, Jolles J. The relation between global and limbic brain volumes on $\mathrm{MRI}$ and cognitive performance in healthy individuals across the age range. Neurobiol Aging. 2000; 21:569-76.

32. Mackay CE, Roberts N, Mayes AR, Downes JJ, Foster JK, Mann D. An exploratory study of the relationship between face recognition memory and the volume of medial temporal lobe structures in healthy young males. Behav Neurol. 1998;11:3-20.

33. Bigler ED, Tate DF. Brain volume, intracranial volume, and dementia. Invest Radiol. 2001;36:539-46.

34. Kuzniecky RI, Jackson GD. Magnetic Resonance in Epilepsy. Neuroimaging Techniques. 2nd ed. London, United Kingdom: Elsevier; 2005.

35. Press WH, Teukolsky SA, Vetterling WT. Chapter 15, Modelling of Data. In: Press WH, Teukolsky SA, Vetterling WT, al. e, editors. Numerical Recipes in C: The Art of Scientific Computing. Cambridge: Cambridge University Press; 1996:663. 



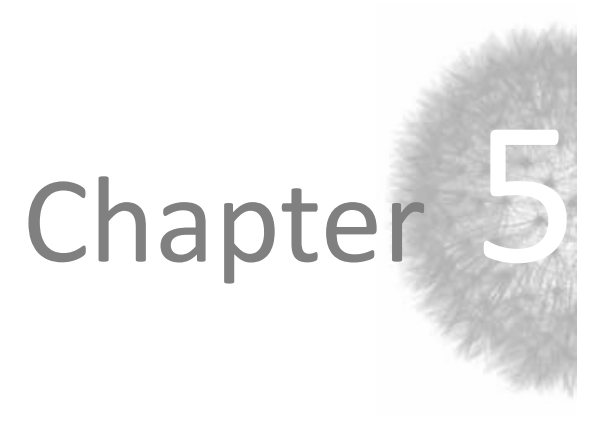

\section{Memory processes and prefrontal network dysfunction in cryptogenic epilepsy}

MCG Vlooswijk, JFA Jansen, CRLPN Jeukens, HJM Majoie, PAM Hofman, MCTFM de Krom, AP Aldenkamp, WH Backes Epilepsia 2011;52:1467-75 


\section{Abstract}

Purpose

Impaired memory performance is the most frequently reported cognitive problem in patients with chronic epilepsy. To examine memory deficits many studies have focused on the role of the mesiotemporal lobe, mostly with hippocampal abnormalities. However, the role of the prefrontal brain remains unresolved. To investigate the neuronal correlates of working memory dysfunction in patients without structural lesions, a combined study of neurocognitive assessment, hippocampal and cerebral volumetry, and functional magnetic resonance imaging of temporal and frontal memory networks was performed.

\section{Methods}

Thirty-six patients with cryptogenic localization-related epilepsy and 21 healthy controls underwent neuropsychological assessment of intelligence (IQ) and memory. On T1-weighted images obtained by 3.0 Tesla MRI, volumetry of the hippocampi and the cerebrum was performed. Functional MRI was performed with a novel picture encoding and Sternberg paradigm that activated different memory-mediating brain regions. Functional connectivity analysis comprised cross-correlation of signal time-series of the most strongly activated regions involved in working memory function.

\section{Results}

Patients with epilepsy displayed lower IQ values, impaired transient aspects of information processing - as indicated by lower scores on the digit-symbol substitution test (DSST), and decreased short-term memory performance than healthy controls - as measured with the WAIS subtests for working memory, and word and figure recognition. This could not be related to any hippocampal volume changes. No group differences were found regarding volumetry or fMRI-derived functional activation. In the Sternberg paradigm, a network involving the anterior cingulate, and the middle and inferior frontal gyrus was activated. A reduced strength of four connections in this prefrontal network was associated with the DSST and word recognition performance in the patient group.

\section{Discussion}

Deficits in the processes involved in transient working memory, and to a lesser extent in short-term memory, in patients with localization-related epilepsy of both temporal and extratemporal origin cannot be attributed to hippocampal atrophy or function only, but are also related to reduced functional connectivity in the prefrontal brain. As patients with symptomatic lesions or mesiotemporal sclerosis were excluded from this study, the results cannot be explained by structural lesions. Therefore, the current findings highlight the influence of epilepsy on the prefrontal network integrity as a possible underlying problem of memory impairment. 


\section{Introduction}

In clinical practice, patients with chronic epilepsy commonly develop co-morbid cognitive problems, ranging from memory deficits and mental slowing, to global cognitive deterioration. ${ }^{1}$ For memory in particular, several aspects can be disturbed, including verbal and figural encoding, working memory, and long-term memory. ${ }^{2}$

Different clinical factors contribute to cognitive impairment in epilepsy, such as antiepileptic drugs (AED), ${ }^{3}$ interictal epileptic discharges, ${ }^{4}$ and severity of seizures. ${ }^{5,6}$ However, the impact on cognition of these factors can be highly variable. A comprehensible model for the development of memory impairment in general in epilepsy is therefore still lacking.

A more rewarding approach may be the investigation of cerebral mechanisms that could be responsible for memory dysfunction. Such mechanisms may be the mediator between epilepsy factors and the development of memory impairment. Usually, this is studied by attempting to find an association with macrostructural abnormalities on MRI, for example by focusing on hippocampal volumes, as hippocampal sclerosis and atrophy are related to memory impairment. Studies using functional MRI (fMRI) are often integrated in the work-up procedure for epilepsy surgery and focused on patients with medically refractory temporal lobe epilepsy (TLE), generally with unilateral hippocampal or mesiotemporal abnormalities. ${ }^{7-11}$

In clinical practice though, memory problems are reported not only by patients with unilateral TLE, but also by those with extratemporal lobe epilepsy and by patients without any structural lesions. These observations suggest that (i) dysfunction of structures outside the mesiotemporal lobe may cause memory deficits, and (ii) memory problems are not solely attributable to structural cerebral lesions.

Based on the 'model of working memory' proposed by Baddeley and Hitch ${ }^{12}$ the term working memory covers the very early, transient aspects of information processing. Central components of the working memory model are the 'central executive', referring to the supervisory system which controls the flow of information from and to its slave systems: the 'phonological loop' (a transient verbal storage system), the 'visuo-spatial sketchpad' (transient visuo-spatial storage system), and the 'episodic buffer' (which links the working memory to more stable phases of the short-term memory system) (Figure 5.1). Theoretically, the cerebral regions involved in the central executive function, may well be located outside the temporal lobe, most likely in the prefrontal regions. Since the central executive serves as a gateway to other, more stable memory processes, its impairment can lead to deficits in short-term memory deficits as well. 


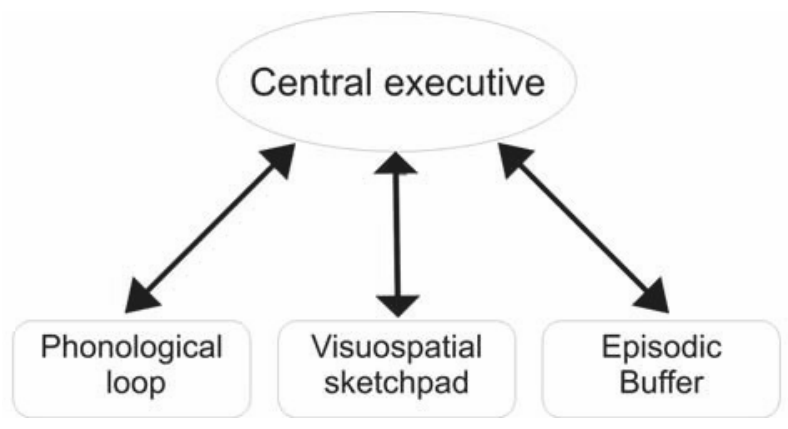

Figure 5.1 Schematic representation of the model of working memory proposed by Baddeley and Hitch ${ }^{12}$

Conventional MRI does not seem suited for detection of early changes associated with cognitive decline. However, with newer MR techniques, more subtle cerebral changes can be investigated. With $\mathrm{fMRI}$, for instance, functional activation patterns during a cognitive task can be localized. As mentioned before, most fMRI studies describe group differences in activation patterns in homogeneous populations with TLE. A disruption of the memory network, independently of whether the temporal or extratemporal nodes in this network are dysfunctional, might result in the same types of memory impairment. With functional connectivity analysis, the integrity of such networks can be investigated.

The aim of the current study was to investigate memory performance, in patients with cryptogenic epilepsy of both temporal and extratemporal origin as compared to controls and to explore the relation with brain volume and function. To assess brain function, we have investigated the relation of memory performance and functional connectivity in the hippocampus and the prefrontal networks. This exploration might reveal a possible etiological explanation for the development of memory deficits in patients with cryptogenic localization-related epilepsy.

\section{Methods}

\section{Participants}

Patients with cryptogenic temporal or extratemporal localization-related epilepsy were recruited from the Epilepsy Centre Kempenhaeghe (Heeze, the Netherlands) and the outpatient Neurology Department of the Maastricht University Medical Centre. Cryptogenic was defined by the absence of structural abnormalities on previous 
imaging (mostly 1.5 Tesla MRI) and exclusion of other causes. Other inclusion criteria were: no history of status epilepticus or other underlying disease that potentially causes cognitive impairment. Healthy controls were family members and acquaintances of the patients without a history of brain injury or cognitive problems.

After careful selection (see Figure 5.2), the study population included 36 patients (19 women; mean age 39 years) and 21 healthy controls ( 12 women; mean age 40 years). All subjects gave written informed consent and approval for the study by the local Medical Ethical Commission was obtained.

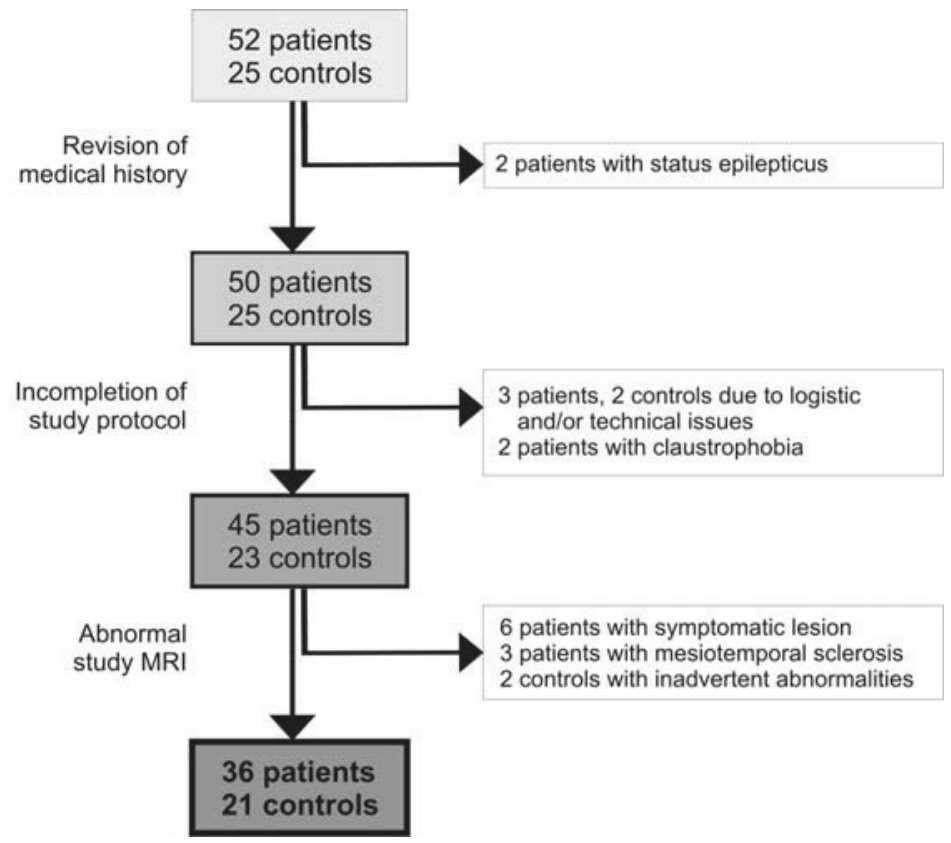

Figure 5.2 Flowchart of inclusion of participants

The following patient data were collected: age at onset of epilepsy, seizure focus, drug load, and total number of partial and secondarily generalized seizures (SGS) during life-time. The latter was calculated using patient records and seizure diaries. Since partial seizures are more prone to occur unperceived and therefore less accurately reported, the estimation of partial seizures is expressed in categories. Drug load was calculated by using the ratio of prescribed daily dose to defined daily dose. ${ }^{13}$ Characteristics of patients and controls are listed in Table 5.1. 
82 Chapter 5

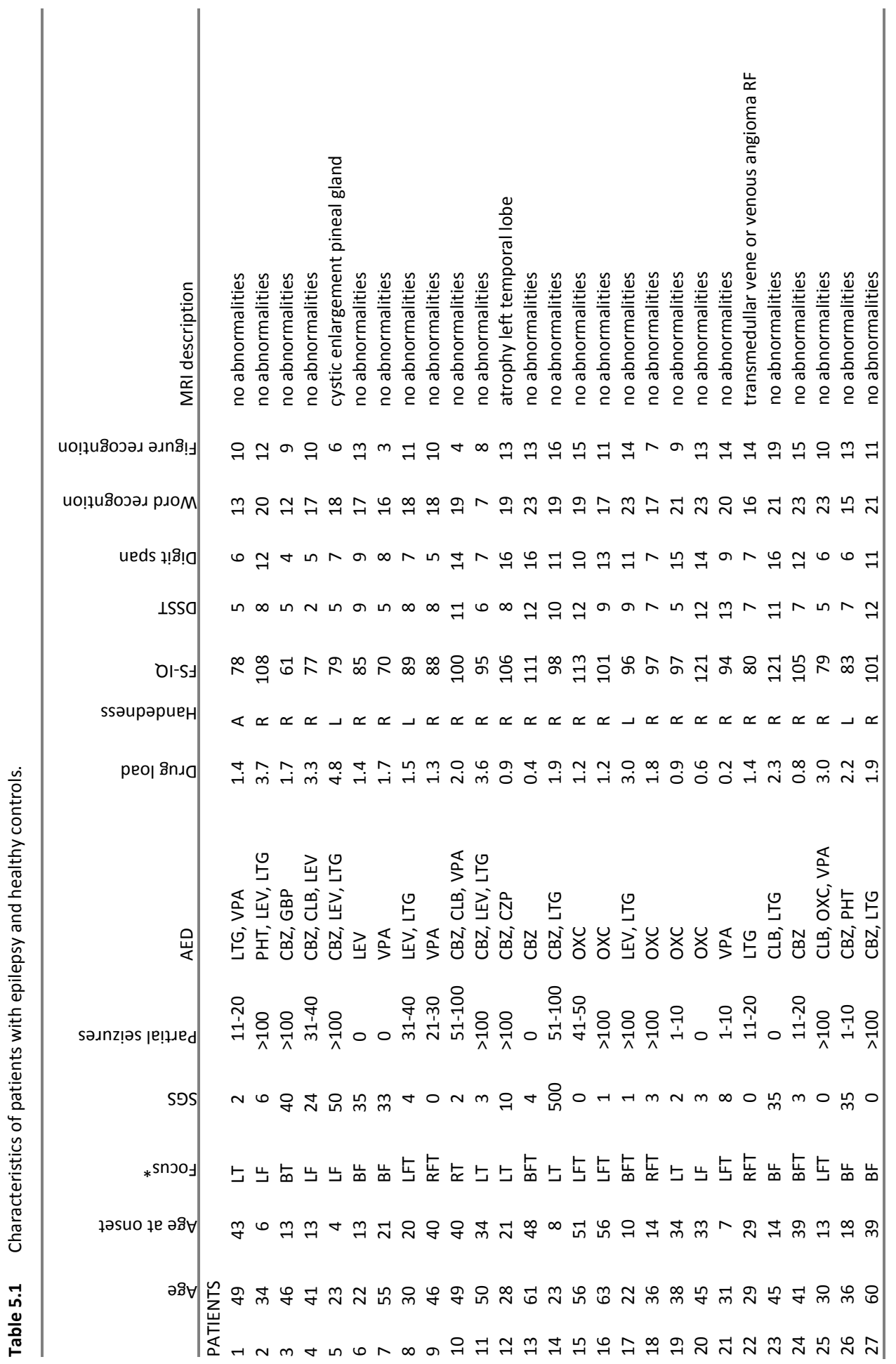




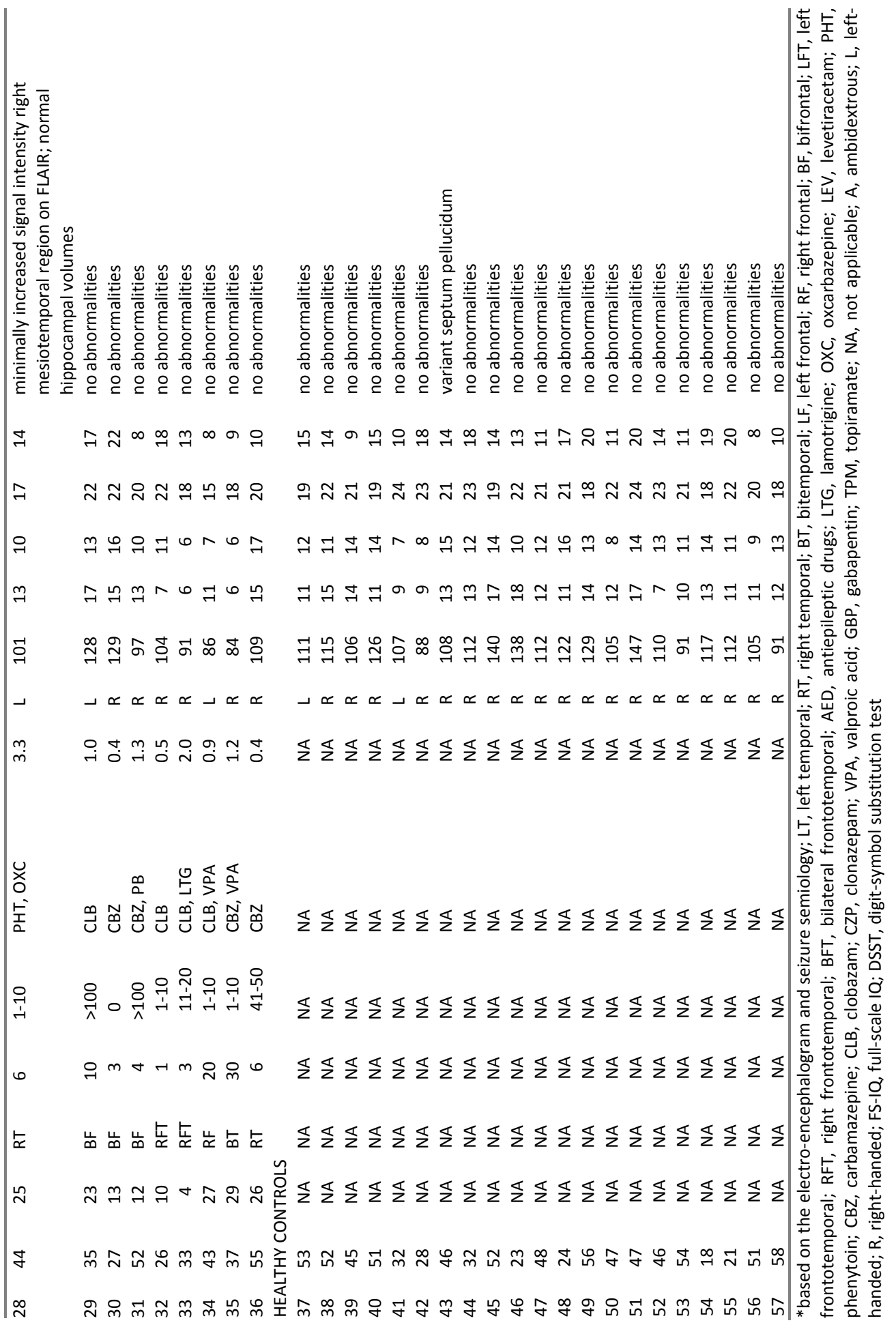




\section{Neurocognitive testing}

All subjects underwent assessment of intelligence and memory. The selection of memory tests was based on assessing those functions that could also be evaluated during $\mathrm{fMRI}$, focusing on working memory and encoding (as necessary for episodic memory). To test intelligence, the Wechsler Adult Intelligence Scale (WAIS-III) was used $^{14}$ with administration of all subtests in the majority of patients. As a measure for the central executive, involved in the early transient memory processes, the WAIS-III subtest digit-symbol substitution test (DSST) was used. ${ }^{15,16}$ For the more stable components of working memory, we used the normalized scores from the WAIS-III subtests included in the Working Memory Index: digit span, letter-number sequencing and arithmetic. Furthermore, two recognition tasks for words and figures from the FePsy computerized neurocognitive test battery ${ }^{17}$ were administered. Handedness

was assessed with the Annett Handedness Questionnaire. ${ }^{18}$ Based on the performance on the DSST $(<9)$ and the word recognition task $(<18)$, two separate groups of cognitively impaired patients were defined. The former consists of 19 impaired patients (vs. 17 unimpaired) and the latter of 12 impaired patients (vs. 24 unimpaired).

\section{fMRI data acquisition}

MRI was performed on a 3.0 Tesla unit (Philips Achieva, Philips Medical Systems, Best, The Netherlands), equipped with an 8-channel head coil. fMRI data were acquired using a whole-brain blood oxygen level-dependent (BOLD) echo-planar imaging sequence, with repetition time $2 \mathrm{~s}$ ( $3 \mathrm{~s}$ for picture encoding), echo time $35 \mathrm{~ms}$, matrix $128 \times 128 \times 32$, field of view (FOV) $256 \times 256 \mathrm{~mm}^{2}, 4 \mathrm{~mm}$ adjacent transverse slices, and 196 volumes per acquisition. For anatomic reference, a T1-weighted 3D turbo field echo was acquired with the following parameters: TR $9.91 \mathrm{~ms}$, TE $4.6 \mathrm{~ms}$, inversion time $3 \mathrm{~s}$, flip angle $8^{\circ}$, voxel size $1 \times 1 \times 1 \mathrm{~mm}^{3}$, matrix $256 \times 256 \times 200,1 \mathrm{~mm}$ coronal slices.

\section{fMRI activation paradigms}

Two fMRI activation paradigms were selected to investigate different memory networks: picture encoding for activation of the hippocampus and surrounding areas, and Sternberg for activation of (among others) the prefrontal network. For a detailed description of these paradigms, see Appendix 5.1.

\section{Image analysis}

fMRI data analysis was performed in MATLAB (Mathworks, Natick, Massachusetts, USA) using brain activation contrasts (between task performance and baseline) according to the General Linear Model (GLM) as implemented in the statistical parametric mapping software package (SPM2) (Wellcome Department of Cognitive 
Neurology, London, UK). The BOLD images were realigned to correct for head movement, transformed into the standardized stereotactic reference system developed by the Montreal Neurological Institute (MNI) and smoothed with a 6-mm Gaussian kernel. In the GLM, the standard discrete cosine-set was used to correct for (low-frequency) nuisance. Additionally, the time-series were convolved with the haemodynamic response function.

A standard random-effects analysis was performed to explore differences between the epilepsy and control group. Results were thresholded at the $p<0.05$ level (corrected for multiple comparisons). Also connectivity analysis was performed using SPM2. Based on the activation maps of the control group, and the anatomical regions identified in the template $\mathrm{MNI}$ image, standard masks were created to select the regions of interest activated in the picture encoding and Sternberg paradigms. For the hippocampus, individual masks were manually drawn for the volumetric analysis. The regions included were both hippocampi $(H C)$, lingual gyrus ( $L G)$, and anterior cingulate cortex (ACC) for the picture encoding paradigm, and bilateral inferior frontal gyrus (IFG), bilateral middle frontal gyrus (MFG), and ACC for the Sternberg paradigm. The signal change between activation and rest was averaged yielding an average individual BOLD response during both tasks within that region. ${ }^{19}$

For the connectivity analysis, an fMRI signal vector from the regions of interest with the course of signal intensity over the 196 volumes was obtained for every subject. ${ }^{20}$ Each vector was low-pass-filtered using a finite impulse response filter to remove the effect of high-frequency noise. The six motion correction parameters were included in the design matrix as confounders. The correlation coefficients of all signal intensities vectors for all regions were calculated, and transformed using the Fisher-Z transformation. Finally, for the possible connections (six for picture encoding; ten for Sternberg), a mean connectivity Z-value was obtained to compare with neurocognitive, volumetric and epilepsy parameters.

\section{Volumetry}

For all subjects, the hippocampi were manually outlined using the freeware software program MRIcron ${ }^{21}$ by two observers who were blinded to all subject information. The delineation protocol, the hippocampal boundaries and the correction for brain volume are described in detail in Chapter $4 .{ }^{22}$ Hippocampal volumes were calculated by multiplying the number of voxels by the voxel volume $\left(1 \mathrm{~mm}^{3}\right)$. The final volume of the hippocampus was determined as the mean of volumes delineated by the two observers. Brain volume was determined by automated segmentation of the cerebrum in the software package SPM2, reckoning with geometric scaling factors, and comprised the total white and grey matter tissue volume of the cerebrum. 


\section{Statistical analysis}

Clinical, neuropsychological and volumetric data analyses were performed in SPSS (Rel. 16.0.1, SPSS Inc., Chicago, USA). To acquire a standardized mean connectivity value for both fMRI paradigms, Z-scores for all connections were calculated and averaged. Functional connectivity values, including the Z-scores for mean functional connectivity, were compared with the neuropsychological test scores, age at onset and drug load using Pearson correlation. For correlation with seizure frequency, Spearman correlation was performed. Additionally, possible differences between patients with epilepsy and healthy volunteers were assessed using a Student's t-test. Post-hoc analysis included one-way ANOVA assessing possible differences associated with seizure focus (frontal, frontotemporal, temporal focus and healthy volunteers).

\section{Results}

\section{Neurocognitive performance (Table 5.2)}

The patient group as a whole had lower IQ scores than controls, as well as worse performance on all other neuropsychological tests. The results of the included patients were overall in the average range, but there was a broad range of cognitive performance, with a subset of patients with evident cognitive impairment. On the contrary, the range of cognitive test results is limited in the control group, with most subjects performing at or around the average group value.

Table 5.2 Neuropsychological test results for patients and healthy controls.

\begin{tabular}{lccccc}
\hline & \multicolumn{2}{c}{ Patients } & \multicolumn{2}{c}{ Healthy controls } \\
Test & mean \pm SD & range & mean \pm SD & range & $p$-value \\
\hline Full-scale IQ & $96 \pm 16$ & $61-129$ & $114 \pm 16$ & $88-147$ & $<0.01$ \\
Digit-symbol substitution test & $9 \pm 4$ & $2-17$ & $12 \pm 3$ & $7-18$ & $<0.01$ \\
Letter-number sequencing & $10 \pm 4$ & $2-19$ & $12 \pm 3$ & $8-18$ & $<0.01$ \\
Digit span & $10 \pm 4$ & $4-17$ & $12 \pm 2$ & $7-16$ & 0.02 \\
Arithmetic & $10 \pm 3$ & $4-18$ & $12 \pm 3$ & $8-17$ & $<0.01$ \\
Word recognition & $19 \pm 3$ & $7-23$ & $21 \pm 2$ & $18-24$ & $<0.01$ \\
Figure recognition & $12 \pm 4$ & $3-22$ & $14 \pm 4$ & $8-20$ & 0.02 \\
\hline
\end{tabular}

\section{Hippocampal and brain volumetry (Table 5.3)}

The left hippocampus was on average $3-6 \%$ smaller than the right hippocampus in both groups (patients $p<0.01$, controls $p=0.02$ ). Comparing patients with controls, hippocampal volumes were slightly smaller for the patient group, but these differences were not significant ( $p=0.07$ left hippocampus; $p=0.17$ right hippocampus) and were comparable to the interobserver variation $\left( \pm 7 \%{ }^{22}\right)$. Subgroup analysis according to seizure focus did not reveal left-right differences for hippocampal volumes. No correlation between hippocampal volumes or brain volume and neuropsychological test results was found. 
Table 5.3 Hippocampal and intracranial volumes $\left(\right.$ in $\mathrm{cm}^{3}$ ) for patients and healthy controls.

\begin{tabular}{|c|c|c|c|c|c|}
\hline Group & Left $\mathrm{HC} \pm \mathrm{SD}$ & Right $\mathrm{HC} \pm \mathrm{SD}$ & $\mathrm{ICV} \pm \mathrm{SD}$ & Left $\mathrm{HC}_{\text {corr }} \pm \mathrm{SD}$ & Right $\mathrm{HC}_{\text {corr }} \pm \mathrm{SD}$ \\
\hline \multicolumn{6}{|l|}{ Patients vs controls } \\
\hline All patients & $2.965 \pm 0.430$ & $3.141 \pm 0.392$ & $1252 \pm 146$ & $3.007 \pm 0.357$ & $3.182 \pm 0.357$ \\
\hline Healthy controls & $3.182 \pm 0.405$ & $3.294 \pm 0.405$ & $1272 \pm 128$ & $3.182 \pm 0.358$ & $3.294 \pm 0.326$ \\
\hline$p$-value & 0.07 & 0.17 & 0.46 & 0.08 & 0.25 \\
\hline \multicolumn{6}{|c|}{ Patients divided according to side of seizure focus } \\
\hline Left-sided & $3.025 \pm 0.569$ & $3.146 \pm 0.492$ & $1286 \pm 128$ & $2.996 \pm 0.497$ & $3.117 \pm 0.468$ \\
\hline Right-sided & $2.891 \pm 0.410$ & $3.086 \pm 0.359$ & $1173 \pm 131$ & $3.089 \pm 0.237$ & $3.284 \pm 0.195$ \\
\hline Bilateral & $2.967 \pm 0.367$ & $3.163 \pm 0.365$ & $1267 \pm 155$ & $2.977 \pm 0.324$ & $3.174 \pm 0.351$ \\
\hline
\end{tabular}

$\mathrm{HC}=$ hippocampus; $\mathrm{SD}=$ standard deviation; $\mathrm{ICV}=$ total intracranial volume; $\mathrm{HC}_{\text {corr }}=$ hippocampal volume corrected for total intracranial volume. No significant differences between groups based on side of seizure focus, calculated with one-way ANOVA.

\section{fMRI: performance}

Both groups performed well on the fMRI paradigms, as measured by monitoring of the button presses during fMRI. Only on the Sternberg paradigm, patients exhibited significantly longer reaction times than controls $(822 \pm 164 \mathrm{~ms}$ for patients vs. $699 \pm 77 \mathrm{~ms}$ for controls, $p<0.01$ ). Reaction times during picture encoding and error rates on both paradigms were comparable in both groups. These results confirm that both groups could perform the paradigms adequately, and that fMRI results can be interpreted reliably.

\section{fMRI: picture encoding paradigm}

\section{Activation maps (Table 5.4) and functional connectivity}

Significant activation was seen bilaterally in the mesiotemporal, anterior cingulate, visual and visual association cortex, and left thalamus (Figure 5.3DE). No significant differences were found between patients and controls for either activation maps or functional connectivity values (Figure 5.3F).

Correlation of functional connectivity values with neurocognitive memory performance did not reveal any significant results.

\section{fMRI: Sternberg paradigm}

\section{Activation maps (Table 5.4) and functional connectivity}

Significant activation was seen bilaterally in the inferior frontal gyrus, middle frontal gyrus, anterior cingulate, visual association cortex and cerebellum (Figure 5.3AB). No significant differences were found in activation maps or functional connectivity values between patients and controls (Figure 5.3C). 
Table 5.4 Functional activation levels (percentage signal change \pm SD).

\begin{tabular}{lcc}
\hline ROIs & Patients & Healthy controls \\
\hline Picture encoding & & \\
ACC & $0.30 \pm 0.14$ & $0.30 \pm 0.18$ \\
left HC & $0.43 \pm 0.19$ & $0.52 \pm 0.19$ \\
right HC & $0.46 \pm 0.18$ & $0.54 \pm 0.20$ \\
LG & $0.43 \pm 0.25$ & $0.59 \pm 0.23$ \\
Sternberg & & \\
ACC & $3.10 \pm 1.15$ & $2.97 \pm 0.64$ \\
left IFG & $2.15 \pm 0.74$ & $1.97 \pm 0.47$ \\
right IFG & $2.27 \pm 0.94$ & $2.43 \pm 0.64$ \\
left MFG & $2.75 \pm 0.95$ & $2.81 \pm 0.96$ \\
right MFG & $2.74 \pm 1.10$ & $2.59 \pm 0.84$ \\
\hline
\end{tabular}

ROIs = regions of interest; $A C C=$ anterior cingulate cortex; $H C=$ hippocampus; $L G=$ lingual gyrus; IFG = inferior frontal gyrus; MFG = middle frontal gyrus
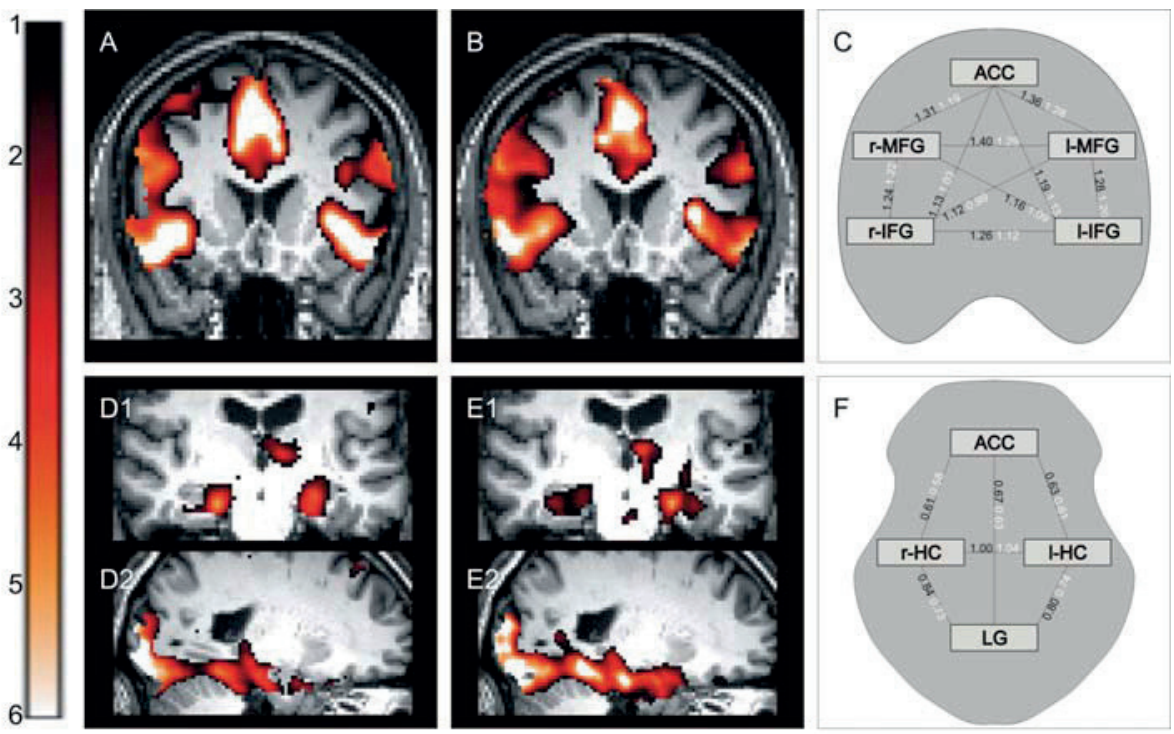

Figure 5.3 Group averaged fMRI activation maps superimposed on a normalized T1-weighted MR image. Mean activation patterns for patients are shown in $A$ and $D$ and for healthy controls in $B$ and E. For the Sternberg paradigm the characteristic bilateral prefrontal network is shown on coronal slices $(A-C)$. For the picture encoding paradigm (D-F) the characteristic activation clusters in the hippocampi and visual association cortex are shown on coronal (D1 and E1) and sagittal (D2 and E2) slices. The selected regions of interest with the functional connectivity values for patients (in black) and controls (in white) for all the connections between these regions are schematically illustrated in a coronal (C) and transverse (F) slice. Connection strengths were not significantly different between the two groups $(p>0.05)$. Locations of the structures on the schematic illustration are not accurate and are positioned for reasons of clarity. The color bar indicates the t-value of the activation level. Slice positions are specified in the MNI coordinate system: $y=16 \mathrm{~mm}$ for $\mathrm{A}$ and $\mathrm{B} ; \mathrm{y}=-20 \mathrm{~mm}$ for $\mathrm{D} 1$ and $\mathrm{E} 1 ; \mathrm{x}=22 \mathrm{~mm}$ for $\mathrm{D} 2$ and $\mathrm{E} 2$. $A C C=$ anterior cingulate cortex; IFG = inferior frontal gyrus; $M F G=$ middle frontal gyrus; $\mathrm{HC}=$ hippocampus; $\mathrm{I}=$ left; $r$ = right; $L G$ = lingual gyrus. 
Correlation of connectivity values with neurocognitive memory performance demonstrated a significant correlation between the digit-symbol substitution test and four of the ten studied connections in the patient group, that is ACC-right MFG $(r=0.34, p=0.04)$, right MFG-left MFG $(r=0.41, p=0.01)$, right MFG-left IFG $(r=0.38$, $p=0.02)$ and left IFG-right IFG $(r=0.35, p=0.04)$ (Figure 5.4A). The impaired patient group (based on DSST scores) displayed significantly lower connections than nonimpaired patients for these four connections $(p<0.036)$.

For two of these connections a significant correlation was demonstrated with the results of the word recognition test (ACC-right MFG, $r=0.35, p=0.04$; and right MFGleft MFG, $r=0.34, p=0.04)$. The connection ACC-left MFG demonstrated a trend towards significant correlation with the word recognition test $(r=0.29, p=0.08)$ (Figure 5.4B). The impaired patient group (based on word recognition scores) displayed significantly lower connections than non-impaired patients for these four connections complemented with the left IFG-right IFG and left IFG-right MFG connections $(p<0.013)$.

In the healthy control group, four of ten connections (ACC-left IFG $(r=0.49, p=0.02)$, ACC-right IFG $(r=0.44, p=0.04)$, ACC-right MFG $(r=0.45, p=0.04)$, left IFG-right MFG $(r=0.44, p=0.05)$, as well as the Z-score for mean functional connectivity $(r=0.45$, $p=0.04$ ) were associated with performance on arithmetic. For the other neuropsychological memory tests no correlations with functional connectivity were found for the healthy controls.
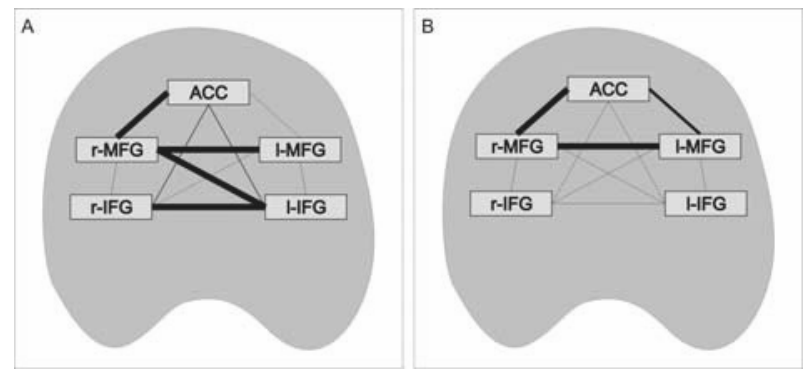

Figure 5.4 Functional connectivity in relation to performance on the digit-symbol substitution test (DSST) (A) and word recognition (B) in patients. The schematic illustration shows the selected regions of interest in the Sternberg paradigm and all possible connections (thin lines). The thick lines indicate the connections which positively correlate with the test scores. The intermediate line indicates a trend towards significant correlation $(p=0.08)$. ACC=anterior cingulate cortex; IFG = inferior frontal gyrus; MFG = middle frontal gyrus; $\mathrm{l}=$ left; $r=$ right.

\section{Clinical parameters}

In the Sternberg paradigm, higher age at onset was correlated with lower functional connectivity for ACC-right MFG $(r=-0.34, p=0.04)$. For the connection right IFG-left MFG, higher values were correlated with higher drug load $(r=0.42, p=0.01)$. No 
other clinical parameters (age, lifetime number of seizures, drug load, side or site of seizure focus) correlated with the fMRI results.

\section{Discussion}

\section{Current findings}

In this study, memory impairment in patients with cryptogenic epilepsy was studied by neurocognitive assessment, volumetry and fMRI. Compared to controls, patients performed worse on all memory tests. Neuropsychological test results of patients ranged from evidently disturbed to normal, with some overlap with results from healthy controls. The memory impairment observed could not convincingly be related to smaller hippocampal or brain volumes. In addition, no significant overall group differences could be demonstrated for functional activation maps or functional connectivity values. Focusing on the patients only, lower functional connectivity values for four of ten possible connections between the activated regions in the Sternberg paradigm was associated with worse performance on the digit-symbol substitution test. This suggests that especially the central executive component of working memory is susceptible for the influence of epilepsy. Moreover, some of these prefrontal connections had lower values with worse performance on word recognition.

\section{Neuronal correlate for working memory dysfunction}

The results of this study confirm the importance of network functionality for working memory functions and the possibility that epilepsy can alter these networks. Interestingly, patients display difficulties in a range of memory functions with poorer performance for both classical (mesio)temporal functions (recognition), as for prefrontal tests (transient and more stable components of working memory). However, these memory deficits could not be linked to hippocampal abnormalities or alterations in the hippocampal network. Instead, in the patient group, a reduction in the synchronicity of cerebral regions within the prefrontal network was associated with dysfunction of the central executive. No comparable correlation could be demonstrated for the functional connectivity values and the tests for short-term memory. One could argue that good functioning of the central executive serves as a gateway for other cognitive functions. The differences in performance on tests for short-term memory are then a consequence of impairments in working memory, specifically in the central executive. However, our data are not sufficient to confirm such an assumption. Since prefrontal functional connectivity decreased with poorer working memory performance, it may reflect a neuropathological process, or at least an insufficient compensatory mechanism. Furthermore, our findings support the 
concept that epilepsy as the underlying condition influences the prefrontal network. This influence does not solely depend on hippocampal abnormalities, since patients with symptomatic lesions (e.g. mesiotemporal abnormalities) were excluded. This notion was further supported by the fact that the network changes did not depend on the side or site of seizure focus.

\section{Comparison with previous studies}

\section{Memory dysfunction}

The finding that patients performed worse on all neurocognitive tests is in keeping with former investigations which demonstrated a variety of memory deficits. ${ }^{23,24}$ Moreover, memory impairment and decline are more pronounced in subgroups with TLE, especially those with lower baseline IQ. ${ }^{24}$ Memory deficits have been described shortly after the occurrence of temporal seizures. ${ }^{25}$ This cannot explain the differences between our patients and controls since seizures did not occur on the day of neurocognitive assessment.

\section{Brain activation}

Studies with patients with mesiotemporal sclerosis ${ }^{26,27}$ or symptomatic lesions such as gliomas, ${ }^{27}$ demonstrated lower cerebral activation as well as poorer memory performance in TLE patients than controls. In patients with hippocampal sclerosis a relative prefrontal hypoactivation and more pronounced activation in the occipitoparietal regions were suggested to be attributable to an alteration of temporoparietal connectivity. ${ }^{28}$ In the current study, no significant between-group differences were found regarding $\mathrm{fMRI}$ activation maps related to memory tasks, which confirms the normal functionality of the hippocampi in the selected patients.

\section{Network integrity}

Our findings that reduced functional connectivity in the engaged network of the patients is associated with poorer working memory performance, is in line with previously demonstrated changes in frontal and temporal networks related to memory dysfunction. However, the novelty of the current study lies in the exclusive selection of patients without any visible lesions on 3.0 Tesla MRI, whereas others have reported on patients with (mesio)temporal MRI abnormalities or mixed patient groups. In a surgical population with unilateral hippocampal sclerosis stronger preoperative intrahemispheric functional connectivity between hippocampus and superior temporal gyrus was associated with better task performance preoperatively, and more verbal learning decline postoperatively. ${ }^{29}$ Stronger preoperative functional connectivity was suggested to indicate higher functional adequacy of the memory network. Poorer quality of autobiographical memory in patients with TLE was reported in combination with a down-regulation of cerebral activity in the entire 
autobiographical memory network. ${ }^{30}$ With functional connectivity analysis an alteration of this network was demonstrated, with bypassing of the left hippocampus and increasing connection strengths between other surrounding areas. Other recent studies in lesional TLE have demonstrated reduced functional connectivity in the socalled default network, ${ }^{31}$ between bilateral medial temporal lobes and left orbitofrontal gyrus ${ }^{32}$ and a shift of levels of functional connectivity from the ipsilateral to the contralateral temporal region. ${ }^{33}$ In the latter study working memory performance correlated with functional connectivity between anterior and posterior parts of the right hippocampus. Impairment of functional connectivity networks has also been demonstrated in language networks ${ }^{20}$ and correlated with language impairment. ${ }^{34}$ In general, network integrity appears to be important for more cognitive functions than memory alone, such as for language and information processing. In this study, this is reflected by the association of performance on the DSST - a test with engagement of multiple cognitive processes reflecting especially the central executive component of working memory - and the integrity of the prefrontal network.

\section{Relation with clinical observations}

In this study, patients were included independent of whether they experienced memory problems or not. Although the patient group as a whole performed evidently worse on all memory tests, patients' performance ranged from obviously disturbed to completely normal. It would have been interesting to relate the objective memory tests and fMRI results to subjective memory appraisal. In clinical practice, many patients complain about memory, but detailed neurocognitive assessment does not always demonstrate clear-cut memory deficits. Several explanations for the perception of poor cognitive skills have been proposed, such as the perception of gaps in experience due to amnesia in ictal and postictal periods, fluctuations in medication levels, and the high incidence of depression in refractory epilepsy. ${ }^{35,36}$ Unfortunately, in this study no objective measures for depression were registered. Future studies should include this. Previous studies have demonstrated that other cognitive functions, such as language skills and access to vocabulary, are also likely to be involved in subjective memory problems. ${ }^{37}$ Another clinical impression is that many patients with memory problems exhibit mental slowing. These observations potentially support the concept that principally the prefrontal networks are dysfunctional, with interruption of cognitive functions which are commonly localized in the prefrontal regions.

\section{Methodological issues}

In the current study we found that reduced functional connectivity in a verbal working memory (Sternberg) paradigm negatively correlated with reduced scores on the DSST test in patients with epilepsy, but not in healthy controls. Therefore this effect 
appeared to be disease specific. For the arithmetic test the opposite was found: a positive correlation between the functional connectivity and the arithmetic scores in the healthy controls, but not in the patients. The absence of any significant effect in the patients cannot be inferred as disease specific. The presence or absence of correlations between the functional connectivity and specific neuropsychological tests needs to be investigated in more detail. In this study only two memory paradigms were utilized for the $\mathrm{fMRI}$ and six tests were used in the neuropsychological exam. Further research is required utilizing more specific memory paradigms to reveal which regional functional connections in the brain explain the impairment of certain memory processes and the nature of the relation between neuropsychological performance and the strength of functional connections in healthy control subjects.

\section{Conclusions}

This study suggests an important role for functional connectivity in the prefrontal networks on working memory performance in patients with epilepsy. For the first time, a correlation between prefrontal network changes and working memory was demonstrated in patients with both temporal and extratemporal epilepsy without symptomatic lesions or mesiotemporal sclerosis. Our findings stress the influence of epilepsy as such on prefrontal network integrity regardless of a structural lesion. Whether there is a causal relationship between epilepsy, prefrontal network dysfunction and memory deficits, or all these entities are the result of yet another still unknown - pathological mechanism, remains to be elucidated. 


\section{Appendix 5.1. Description of fMRI paradigms}

In the picture encoding task, subjects viewed five abstract images of real-life outdoor scenes during anatomic MR imaging. Each picture was presented 25 times for 3 seconds. Approximately one hour later, an MRI examination was performed while variable-length epochs of new pictures (new condition) were demonstrated mixed among variable-length epochs of the five old pictures (old condition). The epochs were mixed into a run of 190 stimuli. With each picture, the subjects indicated whether it was old or new pushing the "OLD" or the "NEW" button. Their decisions and reaction times were automatically recorded. The activation in the new condition was contrasted with the activation in the old condition for further analysis. Previous studies with this paradigm demonstrated activation in memory-related mesial occipital or visual association cortex (Brodmann's area (BA) 17/18), left lateral frontal cortex (BA 44/6/9), bilateral hippocampus, parahippocampal gyrus (BA 35/36) and fusiform gyrus. ${ }^{38,39}$

The Sternberg letter recognition paradigm is a choice-reaction test reflecting verbal working memory performance. ${ }^{40}$ A set of letters was visually presented to maintain in working memory. Subsequently, single letters were presented, and subjects pressed one of two buttons to indicate whether the letter was old or new. In the baseline condition, subjects focused on a cross-hair. The paradigm consisted of 16 blocks (memory set of 1-3 letters for $4 \mathrm{~s}$ followed by 13 response letters of $2 \mathrm{~s}$ each) alternating with 16 baseline rest condition blocks (30s each). The contrast between activation in baseline condition and the two loads was used for further analysis. ${ }^{41}$ In previous studies, prefrontal and temporal areas were activated by this paradigm. ${ }^{42}$ 


\section{References}

1. Oyegbile TO, Dow C, Jones J, Bell B, Rutecki P, Sheth R, Seidenberg M, Hermann BP. The nature and course of neuropsychological morbidity in chronic temporal lobe epilepsy. Neurology. 2004;62: 1736-42.

2. Helmstaedter C. Effects of chronic epilepsy on declarative memory systems. Prog Brain Res. 2002;135:439-53.

3. Jokeit H, Kramer G, Ebner A. Do antiepileptic drugs accelerate forgetting? Epilepsy Behav. 2005;6: 430-2.

4. Aldenkamp A, Arends J. The relative influence of epileptic EEG discharges, short nonconvulsive seizures, and type of epilepsy on cognitive function. Epilepsia. 2004;45:54-63.

5. Thompson PJ, Duncan JS. Cognitive decline in severe intractable epilepsy. Epilepsia. 2005;46:1780-7.

6. Dodrill CB. Progressive cognitive decline in adolescents and adults with epilepsy. Prog Brain Res. 2002;135:399-407.

7. Powell HW, Richardson MP, Symms MR, Boulby PA, Thompson PJ, Duncan JS, Koepp MJ. Preoperative fMRI predicts memory decline following anterior temporal lobe resection. J Neurol Neurosurg Psychiatry. 2008;79:686-93.

8. Binder JR, Sabsevitz DS, Swanson SJ, Hammeke TA, Raghavan M, Mueller WM. Use of preoperative functional MRI to predict verbal memory decline after temporal lobe epilepsy surgery. Epilepsia. 2008;49:1377-94.

9. Baxendale S, Thompson PJ, Duncan JS. Improvements in memory function following anterior temporal lobe resection for epilepsy. Neurology. 2008;71:1319-25.

10. Crane J, Milner B. What went where? Impaired object-location learning in patients with right hippocampal lesions. Hippocampus. 2005;15:216-31.

11. Cheung MC, Chan AS, Lam JM, Chan YL. Pre- and postoperative $\mathrm{FMRI}$ and clinical memory performance in temporal lobe epilepsy. J Neurol Neurosurg Psychiatry. 2009;80:1099-106.

12. Baddeley AD, Hitch G. Working memory. In: Bower GA, editor. Psychology of Learning and Motivation. New York: Academic Press; 1974:47-89.

13. Lammers MW, Hekster YA, Keyser A, Meinardi H, Renier WO, van Lier H. Monotherapy or polytherapy for epilepsy revisited: a quantitative assessment. Epilepsia. 1995;36:440-6.

14. Wechsler D. Manual for the Wechsler Adult Intelligence Scale-III. San Antonio: The Psychological Corporation; 1997.

15. Byrne MD. Taking a computational approach to aging: the SPAN theory of working memory. Psychology and aging. 1998;13:309-22.

16. Pukrop R, Matuschek E, Ruhrmann S, Brockhaus-Dumke A, Tendolkar I, Bertsch A, Klosterkotter J. Dimensions of working memory dysfunction in schizophrenia. Schizophrenia research. 2003;62: 259-68.

17. Alpherts WCJ, Aldenkamp AP. FePsy: The iron psyche. Heemstede; 1994.

18. Annett M. A classification of hand preference by association analysis. Br J Psychol. 1970;61:303-21.

19. Riecker A, Grodd W, Klose U, Schulz JB, Groschel K, Erb M, Ackermann H, Kastrup A. Relation between regional functional $\mathrm{MRI}$ activation and vascular reactivity to carbon dioxide during normal aging. J Cereb Blood Flow Metab. 2003;23:565-73.

20. Waites AB, Briellmann RS, Saling MM, Abbott DF, Jackson GD. Functional connectivity networks are disrupted in left temporal lobe epilepsy. Ann Neurol. 2006;59:335-43.

21. Rorden C. http://www.sph.sc.edu/comd/rorden/mricron/. 2007

22. Jeukens CRLPN, Vlooswijk MCG, Majoie HJM, de Krom MCTFM, Aldenkamp AP, Hofman PAM, Jansen JFA, Backes WH. Hippocampal MRI volumetry at 3 Tesla: reliability and practical guidance. Invest Radiol. 2009;44:509-17.

23. Bengner T, Malina T, Lindenau M, Voges B, Goebell E, Stodieck S. Face memory in MRI-positive and MRI-negative temporal lobe epilepsy. Epilepsia. 2006;47:1904-14.

24. Hermann BP, Seidenberg M, Dow C, Jones J, Rutecki P, Bhattacharya A, Bell B. Cognitive prognosis in chronic temporal lobe epilepsy. Ann Neurol. 2006;60:80-7.

25. Helmstaedter C, Elger CE, Lendt M. Postictal courses of cognitive deficits in focal epilepsies. Epilepsia. 1994;35:1073-8. 
26. Dupont S, Samson Y, Van de Moortele PF, Samson S, Poline JB, Hasboun D, Le Bihan D, Baulac M. Bilateral hemispheric alteration of memory processes in right medial temporal lobe epilepsy. J Neurol Neurosurg Psychiatry. 2002;73:478-85.

27. Cheung MC, Chan AS, Chan YL, Lam JM, Lam W. Effects of illness duration on memory processing of patients with temporal lobe epilepsy. Epilepsia. 2006;47:1320-8.

28. Engelsen BA, Gramstad A, Thomsen T, Beneventi H, Ersland L, Smievoll Al, Lundervold A, Hugdahl K. Frontoparietal activation during delayed visuospatial recall in patients with epilepsy due to hippocampal sclerosis. Epilepsy Behav. 2006;8:565-74.

29. Wagner K, Frings L, Halsband U, Everts R, Buller A, Spreer J, Zentner J, Schulze-Bonhage A. Hippocampal functional connectivity reflects verbal episodic memory network integrity. Neuroreport. 2007;18:1719-23.

30. Addis DR, Moscovitch M, McAndrews MP. Consequences of hippocampal damage across the autobiographical memory network in left temporal lobe epilepsy. Brain. 2007;130:2327-42.

31. Frings L, Schulze-Bonhage A, Spreer J, Wagner K. Remote effects of hippocampal damage on default network connectivity in the human brain. J Neurol. 2009;256:2021-9.

32. Voets NL, Adcock JE, Stacey R, Hart Y, Carpenter K, Matthews PM, Beckmann CF. Functional and structural changes in the memory network associated with left temporal lobe epilepsy. Hum Brain Mapp. 2009;30:4070-81.

33. Bettus G, Guedj E, Joyeux F, Confort-Gouny S, Soulier E, Laguitton V, Cozzone PJ, Chauvel P, Ranjeva JP, Bartolomei F, Guye M. Decreased basal fMRI functional connectivity in epileptogenic networks and contralateral compensatory mechanisms. Hum Brain Mapp. 2009;30:1580-91.

34. Vlooswijk MCG, Jansen JFA, Majoie HJM, Hofman PAM, de Krom MCTFM, Aldenkamp AP, Backes WH. Functional connectivity and language impairment in cryptogenic localization-related epilepsy. Neurology. 2010;75:395-402.

35. Selwa LM, Berent S, Giordani B, Henry TR, Buchtel HA, Ross DA. Serial cognitive testing in temporal lobe epilepsy: longitudinal changes with medical and surgical therapies. Epilepsia. 1994;35:743-9.

36. Thompson PJ, Corcoran R. Everyday memory failures in people with epilepsy. Epilepsia. 1992;33 Suppl 6:S18-20.

37. Helmstaedter C, Elger CE. Behavioral markers for self- and other-attribution of memory: a study in patients with temporal lobe epilepsy and healthy volunteers. Epilepsy Res. 2000;41:235-43.

38. Deblaere K, Backes WH, Tieleman A, Vandemaele P, Defreyne L, Vonck K, Hofman P, Boon P, Vermeulen J, Wilmink J, Aldenkamp A, Boon PA, Vingerhoets G, Achten E. Lateralized anterior mesiotemporal lobe activation: semirandom functional MR imaging encoding paradigm in patients with temporal lobe epilepsy--initial experience. Radiology. 2005;236:996-1003.

39. Deblaere K, Backes WH, Hofman P, Vandemaele P, Boon PA, Vonck K, Boon P, Troost J, Vermeulen J, Wilmink J, Achten E, Aldenkamp A. Developing a comprehensive presurgical functional MRI protocol for patients with intractable temporal lobe epilepsy: a pilot study. Neuroradiology. 2002;44:667-73.

40. Sternberg S. High-speed scanning in human memory. Science. 1966;153:652-4.

41. Vlooswijk MCG, Jansen JFA, de Krom MCTFM, Majoie HJM, Hofman PAM, Backes WH, Aldenkamp AP. Functional $\mathrm{MRI}$ in chronic epilepsy: associations with cognitive impairment. Lancet Neurol. 2010;9:1018-27.

42. Hillary FG, Chiaravalloti ND, Ricker JH, Steffener J, Bly BM, Lange G, Liu WC, Kalnin AJ, DeLuca J. An investigation of working memory rehearsal in multiple sclerosis using fMRI. Journal of Clinical and Experimental Neuropsychology. 2003;25:965-78. 


\section{Chapter}

Functional connectivity and language impairment in cryptogenic localization-related epilepsy

MCG Vlooswijk, JFA Jansen, HJM Majoie, PAM Hofman, MCTFM de Krom, AP Aldenkamp, WH Backes

Neurology 2010;75:395-402 


\section{Abstract}

\section{Background}

An often underestimated cognitive morbidity in patients with epilepsy is language dysfunction. To investigate the neuronal mechanisms underlying neuropsychological language impairment, activation maps and functional connectivity networks were studied by fMRI of language.

\section{Methods}

Fifty-two patients with cryptogenic localization-related epilepsy and 27 healthy controls underwent neuropsychological assessment of $\mathrm{IQ}$, word fluency, and text reading. $\mathrm{fMRI}$ was performed with a standard covert word generation and text reading paradigm. Functional connectivity analysis comprised crosscorrelation of signal timeseries of the characteristic and most strongly activated regions involved in the language tasks.

\section{Results}

After careful selection, 34 patients and 20 healthy controls were found eligible for analysis. Patients displayed lower IQ, lower fluency word count, and lower number of words correctly read compared to controls. fMRI activation maps did not differ significantly between patients and controls. For the word generation paradigm, patients with epilepsy had significantly lower functional connectivity than controls in the prefrontal network. Patients performing worse on the word fluency test demonstrated a significantly lower mean functional connectivity than controls. Text reading demonstrated lower functional connectivity in patients with epilepsy in the frontotemporal network. Similarly, lower mean functional connectivity was observed in patients with lowest reading performance compared to controls. A relation between reduced functional connectivity and performance on word fluency and text reading tests was demonstrated in epilepsy patients.

\section{Conclusion}

Impaired performance on language assessment in epilepsy patients is associated with loss of functional connectivity in the cognitive language networks. 


\section{Introduction}

Patients with epilepsy commonly have cognitive problems, ranging from memory deficits to global cognitive deterioration. ${ }^{1}$ Only modest attention has been given to language dysfunction in chronic epilepsy. Several studies have demonstrated an association between language dysfunction and poorer verbal memory and learning performance in temporal lobe epilepsy (TLE), ${ }^{2-4}$ but also with subjective memory deficits. $^{5}$

Different clinical factors contribute to cognitive impairment in epilepsy, including antiepileptic drugs (AED), ${ }^{6}$ head trauma, ${ }^{7}$ interictal epileptic activity, ${ }^{8}$ status epilepticus, ${ }^{9}$ and even single seizures. ${ }^{7}$ However, for individual patients these factors cannot always explain their cognitive problems, especially in cryptogenic epilepsy.

Cognitive comorbidity in TLE is associated with volumetric abnormalities, such as overall brain atrophy, ${ }^{10,11}$ and atrophy of the left temporal lobe ${ }^{12}$ and corpus callosum. ${ }^{13}$ Also, diffusion-tensor imaging (DTI) demonstrated an association between fiber tract damage and cognitive impairment in TLE. ${ }^{14}$ Most, especially presurgical, studies focus on memory impairment in TLE, demonstrating relations between hippocampal injury and memory deficits. ${ }^{15,16}$ However, memory problems are also reported in extratemporal lobe epilepsy. As subjective memory problems are often related to language dysfunction, ${ }^{5}$ language networks may be similarly affected.

The relation between cognitive function and language organization in epilepsy is complex due to possible intrahemispheric and interhemispheric language reorganization in localization-related epilepsy ${ }^{17-19}$ and highly variable epilepsy characteristics. Moreover, cognitive functions such as language result from interactions of various rather than isolated brain regions. ${ }^{20,21}$

The objectives of this study were to investigate expressive and receptive language performance in cryptogenic localization-related epilepsy and relate language performance to functional connectivity in frontotemporal language networks.

\section{Methods}

\section{Participants}

Inclusion criteria for the patients were confirmed cryptogenic (i.e., nonsymptomatic) localization-related epilepsy with an epileptic focus in the frontal or temporal lobes, no history of status epilepticus, and no other disease that could cause cognitive decline. Healthy controls were family members and acquaintances of the patients 
without a history of brain injury or cognitive problems. All subjects gave written informed consent. Approval for the study by the local Medical Ethical Committee was obtained.

Initially, 52 patients and 27 controls were included, of whom 18 patients and 7 controls were excluded afterwards. Reasons for exclusion were status epilepticus in history $(n=2)$, symptomatic lesion detected by 3.0 Tesla MRI examinations ( $n=6)$, claustrophobia ( $\mathrm{n}=2$ ), decline of participation (6 patients, 5 controls), incomplete neuropsychological assessment ( 2 patients), and abnormal MRI in controls ( $n=2)$. The final study population included 34 patients ( 18 women and 16 men; mean age 40 years, range $22-63$; side of seizure focus: left $n=15$, right $n=6$, bilateral $n=13$; focus location: frontal $n=13$, temporal $n=8$, frontotemporal $n=11 ; 8$ left-handed, 1 ambidextrous) and 20 healthy controls ( 11 women and 9 men; mean age 40 years, range $18-56 ; 2$ left-handed).

The following patient data were collected (for more details, see Table 6.1: age at onset (mean 22 years, range $4-56$ ), total number of secondarily generalized seizures (SGS) experienced and partial seizures during lifetime, current seizure type, current seizure frequency (averaged over the last 12 months), seizure focus, and drug load. Total number of SGS was calculated according to the patient record and seizure diaries. For those patients with relatively low numbers of SGS, the exact number could be withdrawn from the patient record. For those with relatively high numbers of SGS (approximately 20 or more), the number was calculated according to the seizure frequency during subsequent periods, correcting for changes in seizure frequency (for example: weekly seizures during a few months followed by a period of seizurefreedom). Drug load was calculated by the ratio of prescribed daily dose to defined daily dose. ${ }^{22}$

\section{Neuropsychological testing}

For intelligence, the Wechsler Adult Intelligence Scale-III was used. ${ }^{23}$ For handedness, the Annett Handedness Questionnaire was administered. ${ }^{24}$ In a clinical test for word fluency, the participants were asked to name as many animals as possible in 2 minutes (Stichting Afasie Nederland word fluency test. ${ }^{25}$ The discrimination level between normal and abnormal performance was set at the mean score for controls -2 SD. For text reading, the participants were instructed to overtly read a meaningful text (about Dutch sparrows) as quickly as possible. The words correctly read during 1 minute were scored. Poor performance was defined as a text reading score below the median of all patients' scores. 


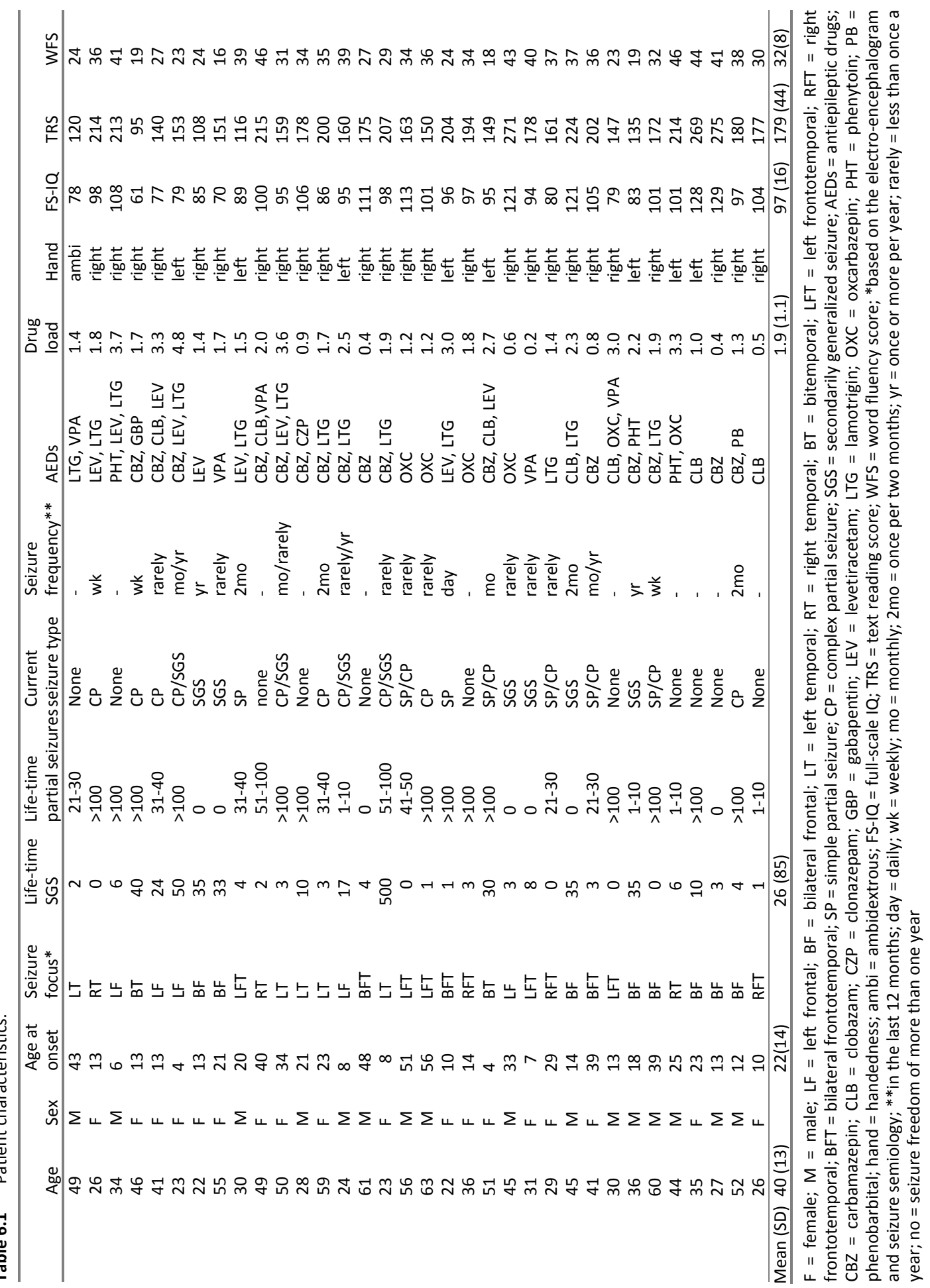




\section{MRI protocol}

All subjects underwent a clinical epilepsy protocol comprising isotropic T1-weighted, fluid-attenuated inversion recovery, inversion recovery, and T2-weighted MRI.

MRI was performed on a 3.0 Tesla unit equipped with an 8-channel head coil. Functional MRI data were acquired using a whole-brain single-shot 3-dimensional blood oxygen level-dependent (BOLD) echoplanar imaging pulse sequence, with repetition time (TR) $2 \mathrm{~s}$, echo time (TE) $35 \mathrm{~ms}$, flip angle $90^{\circ}$, voxel size $2 \times 2 \times 4 \mathrm{~mm}^{3}$, and 196 volumes per acquisition. For anatomic reference, a T1-weighted threedimensional fast gradient echo was acquired with TR $9.91 \mathrm{~ms}$, TE $4.6 \mathrm{~ms}$, inversion time $3 \mathrm{~s}$, flip angle $8^{\circ}$, and voxel size $1 \times 1 \times 1 \mathrm{~mm}^{3}$.

\section{fMRI activation paradigms}

In the word generation paradigm, subjects had to covertly generate as many words as possible starting with a visually presented letter (U-N-K-A-E-P). The paradigm consisted of 6 word generation condition blocks (1 letter per $30 \mathrm{~s}$ block) alternated with baseline rest condition blocks (30 s). Afterwards, all subjects were able to sufficiently reproduce words generated during the task. Previous studies have demonstrated activation in the anterior cingulate and inferior and middle prefrontal cortex. ${ }^{26,27}$

The reading paradigm consisted of a meaningful text with semantic content ( 6 blocks of $30 \mathrm{~s}$ each) which was alternated with nonsense words (7 blocks of $30 \mathrm{~s}$ each). Afterwards, all subjects were able to give a summary of the meaningful text. In previous studies using this reading paradigm, activation of the bilateral fusiform gyrus, middle temporal gyrus (MTG), and anterior temporal pole was observed. ${ }^{26,27}$

\section{Image analysis}

Image preprocessing. Analysis of the time-series data was performed in the Statistical Parametric Mapping (SPM2) software application (Wellcome Department of Cognitive Neurology, London, UK). Dynamic images were slice-timed and realigned to correct for head movement. The corrected images were transformed into the standardized stereotactic coordinate system developed by the Montreal Neurological Institute and smoothed (6-mm kernel).

Activation and functional connectivity analysis. Brain activation was assessed in terms of activation contrast between the task and baseline condition according to the General Linear Model in SPM2. A simple standard random-effects analysis was performed to assess differences in cerebral activation between the groups thresholded at the $p<0.05$ level, corrected for multiple comparisons. ${ }^{28}$ First, the 
activation maps of the 2 groups were compared on a pixel-by-pixel basis and clusters of significantly (family-wise error corrected) activated brain regions were reported. Second, based on the activation maps of the control group, masks were created to select the regions of interest significantly activated. The average individual BOLD response value was expressed as percentage signal change. ${ }^{29}$

The selected brain regions for further analysis were based on the results of previous studies in healthy subjects using the same language paradigms. ${ }^{26,27}$ These regions included for the word generation paradigm left and right inferior frontal gyrus (IFG), left middle frontal gyrus (MFG), and the dorsal part of anterior cingulate cortex (ACC). For the reading paradigm, the regions of interest included left and right MTG, left IFG, and ACC. Within each of these regions, for each subject the 200 voxels with the highest $t$-value were selected to exclude noisy signal time-courses and to focus the assessment of the connectivity of activated brain regions.

For the functional connectivity analysis, the signal time-courses were obtained similar to the procedure described by other investigators. ${ }^{30}$ Time-course data were low-passfiltered to remove the effect of high-frequency signal components corrected for motion effects by using the 6 motion correction parameters as covariates. The correlation coefficients between all regions were calculated using the Fisher- $Z$ transformation. ${ }^{31}$ Finally, for the resulting 6 possible interregional connections, a mean functional connectivity value was calculated per subject.

\section{Statistical analysis}

Statistical data analyses were performed in SPSS (SPSS Inc., Chicago, Illinois, USA). Relevant values were expressed as mean value \pm SD. Mean functional connectivity values were correlated (Pearson) with word fluency and text reading performance. Differences between patients with epilepsy and healthy controls were assessed using Student $t$-tests.

First, differences between patients and controls were assessed for each fMRI paradigm. Second, the functional connectivity for each task was correlated with neuropsychological test results. Third, for both tasks, the functional connectivity values of the patients with poorest neuropsychological test performance were compared with those of controls. To correct for multiple comparisons, a false discovery rate of 0.05 was applied. ${ }^{32}$

Using a one-way analysis of variance test, the potential effect of side of seizure focus on functional connectivity was investigated with 4 groups: patients with left temporal or frontotemporal focus, patients with right temporal or frontotemporal focus, patients with frontal or bilateral (fronto)temporal focus, and healthy controls. 


\section{Results}

\section{Neuropsychological testing}

Patients with epilepsy displayed lower IQ ( $97 \pm 16$, median \pm SD), compared to healthy controls $(113 \pm 15, p<0.01)$. The performance on the word fluency test was also worse than controls ( $32 \pm 8$ for patients and $43 \pm 9$ for controls; $p<0.01$ ). Patients read less words than controls ( $179 \pm 44$ and $207 \pm 34 ; p=0.02$ ). A correlation between performance on word fluency and text reading tests was demonstrated for patients $(r=0.66, p<0.01)$ and controls $(r=0.51, p=0.02)$.

\section{fMRI of word generation}

fMRI and the corresponding statistical results are summarized in Table 6.2.

Table 6.2 fMRI results for patients with epilepsy and controls.

\begin{tabular}{|c|c|c|c|}
\hline & $\begin{array}{c}\text { Patients } \\
\text { (mean } \pm \text { SD) }\end{array}$ & $\begin{array}{c}\text { Controls } \\
\text { (mean } \pm \text { SD) }\end{array}$ & $p$-value \\
\hline \multicolumn{4}{|l|}{ Word generation paradigm } \\
\hline $\mathrm{ACC}$ & $1.74 \pm 0.64$ & $1.85 \pm 0.56$ & 0.54 \\
\hline left IFG & $1.67 \pm 0.69$ & $1.90 \pm 0.53$ & 0.22 \\
\hline right IFG & $1.27 \pm 0.56$ & $1.26 \pm 0.44$ & 0.94 \\
\hline left MFG & $1.69 \pm 0.63$ & $2.02 \pm 0.56$ & 0.06 \\
\hline \multicolumn{4}{|l|}{ Functional connectivity } \\
\hline ACC-left IFG & $1.15 \pm 0.28$ & $1.44 \pm 0.18$ & $<0.01^{*}$ \\
\hline ACC-right IFG & $0.95 \pm 0.31$ & $1.10 \pm 0.21$ & 0.05 \\
\hline ACC-left MFG & $1.28 \pm 0.25$ & $1.43 \pm 0.16$ & $0.02 *$ \\
\hline left IFG-right IFG & $0.97 \pm 0.29$ & $1.09 \pm 0.19$ & 0.12 \\
\hline left IFG-left MFG & $1.32 \pm 0.35$ & $1.55 \pm 0.26$ & $0.02 *$ \\
\hline right IFG-left MFG & $0.92 \pm 0.30$ & $0.98 \pm 0.23$ & 0.43 \\
\hline mean connectivity & $1.10 \pm 0.25$ & $1.27 \pm 0.16$ & $0.01^{*}$ \\
\hline \multicolumn{4}{|l|}{ Text reading paradigm } \\
\hline \multicolumn{4}{|l|}{ Signal change (\%) } \\
\hline ACC & $0.55 \pm 0.40$ & $0.42 \pm 0.17$ & 0.18 \\
\hline left IFG & $0.82 \pm 0.52$ & $0.77 \pm 0.29$ & 0.67 \\
\hline left MTG & $0.88 \pm 0.42$ & $0.86 \pm 0.28$ & 0.83 \\
\hline right MTG & $0.61 \pm 0.31$ & $0.60 \pm 0.20$ & 0.91 \\
\hline \multicolumn{4}{|l|}{ Functional connectivity } \\
\hline ACC-left IFG & $0.71 \pm 0.25$ & $0.79 \pm 0.30$ & 0.28 \\
\hline ACC-left MTG & $0.64 \pm 0.19$ & $0.71 \pm 0.30$ & 0.35 \\
\hline ACC-right MTG & $0.56 \pm 0.23$ & $0.65 \pm 0.29$ & 0.26 \\
\hline left IFG-left MTG & $0.88 \pm 0.25$ & $1.16 \pm 0.86$ & $<0.01^{*}$ \\
\hline left IFG-right MTG & $0.66 \pm 0.27$ & $0.89 \pm 0.29$ & $<0.01^{*}$ \\
\hline left MTG-right MTG & $0.91 \pm 0.30$ & $1.11 \pm 0.34$ & 0.03 \\
\hline mean connectivity & $0.73 \pm 0.20$ & $0.88 \pm 0.22$ & $0.01^{*}$ \\
\hline
\end{tabular}

* statistically significant after controlling for false discovery rate; ACC = anterior cingulate cortex; IFG = inferior frontal gyrus; MFG = middle frontal gyrus; MTG = middle temporal gyrus. 
Activation maps of the word generation paradigm revealed significantly activated clusters in the left inferior and left middle frontal cortex (Broca region), the right middle frontal cortex, and the anterior cingulate cortex for both groups. The activation cluster in the left inferior parietal lobule was significant for the patients, but not for the control group. No significant differences were found between controls and patients with epilepsy (Figure 6.1A and 6.1B).

Functional connectivity values for patients with epilepsy were significantly lower than in healthy controls for 3 connections between the 4 selected regions: ACC-left IFG, ACC-left MFG, and left IFG-left MFG (Figure 6.1C). The mean functional connectivity value in patients was also significantly lower than in controls.
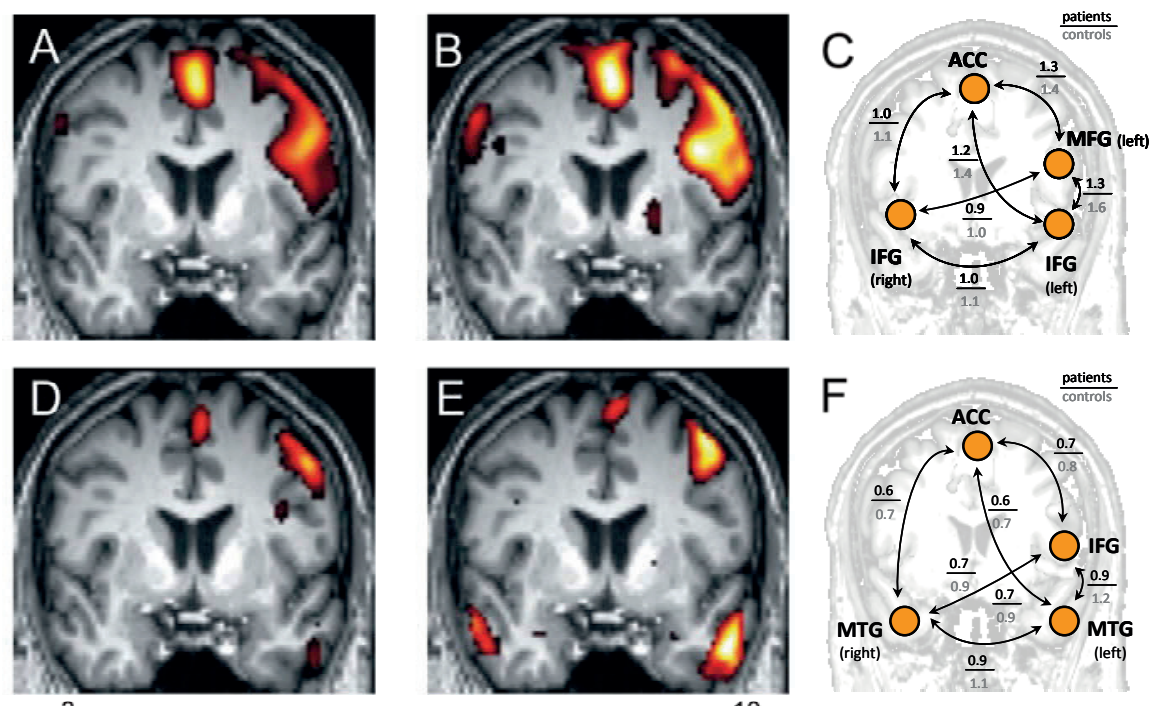

3

10

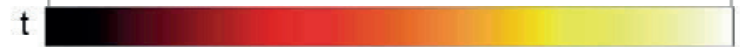

Figure 6.1 Coronal images of group averaged fMRI activation maps superimposed on a normalized T1weighted MR image. Mean activation patterns for patients are shown in $A$ and $D$ and for healthy controls in $\mathrm{B}$ and $\mathrm{E}$. For the word generation paradigm the characteristic bilateral, but left dominant, prefrontal network is shown $(A-C)$. For the text reading paradigm the characteristic activation clusters in the bilateral temporal cortex are shown, with left hemispheric dominance (D-F). The selected regions of interest with the functional connectivity values for patients (in black) and controls (in gray) for all the connections between these regions are schematically illustrated ( $C$ and $F$ ). $A$ thick line in $C$ and $F$ indicates a significant difference between patients and controls. The error bar indicates the $t$-value of the colour-coded activation level. Slice positions are specified in the MNI coordinate system. $A C C=$ anterior cingulate cortex; IFG-I = left inferior frontal gyrus; IFG-r = right inferior frontal gyrus; MFG-I = left middle frontal gyrus; MTG-I = left middle temporal gyrus; $M T G-r=$ right middle temporal gyrus. 
Correlation of functional connectivity with language performance (Table 6.3) demonstrated a significant correlation between mean functional connectivity and word fluency performance in patients. To focus on those patients performing worst on the word fluency test, the patient group was divided in 2 groups based on the performance of the control group (mean-2 SD): 1 subgroup with scores $\leq 34$ words and the other with scores $>34$. When comparing the $\leq 34$ group ( $n=18,53 \%$ ) with controls, the mean functional connectivity value was significantly lower in the patient group (Figure 6.2A). Two (10\%) controls had word fluency scores of 34 or lower.

The relation between mean functional connectivity in word generation and text reading performance demonstrated significantly lower mean functional connectivity with lower reading scores in the patient group. The patient group was divided in 2 groups based on reading scores of $\leq 176$ and $>176$. The reading score of 176 words is the median number of words read by the patients. A comparison between patients with low reading score $(n=17,50 \%)$ and all controls revealed significantly lower mean functional connectivity for the patients. Three $(15 \%)$ controls had a reading score lower than 176.

\section{fMRI of text reading paradigm}

Activation maps of the controls showed significantly activated clusters in the left inferior frontal cortex, the anterior cingulate, and the bilateral temporal cortex. For the patients only the left temporal cortex showed a significantly activated cluster. No significant differences were found between patients and controls (Figure 6.1D and 6.1E).

Functional connectivity values were lower in patients for the connections left IFG-left MTG and left IFG-right MTG. The mean functional connectivity value in patients was significantly lower than in controls (Figure 6.1F).

Correlation of functional connectivity with neuropsychological language performance (Table 6.3) with analysis of text reading performance demonstrated a lower mean functional connectivity value for the patient subgroup with low reading performance (reading score $\leq 176$ ) as compared with controls (Figure 6.2B). A comparison of fMRI results of the text reading paradigm with word fluency performance did not reveal any significant differences for the $\leq 34$ patient group as compared to controls. 


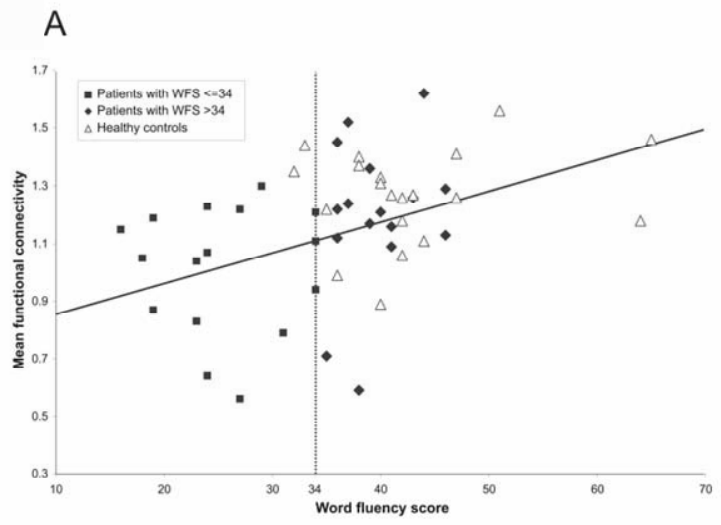

B

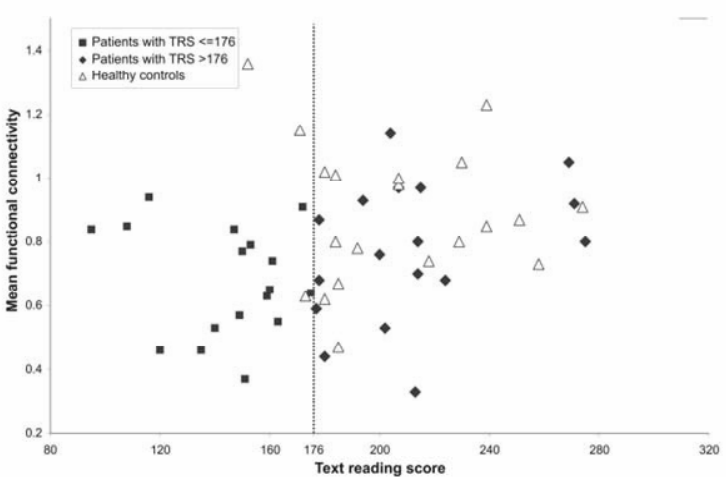

Figure 6.2 (A) Word fluency scores and mean functional connectivity in the word generation fMRI paradigm. Correlation between word fluency scores and mean functional connectivity was significant for the entire patient group as indicated by the (solid) regression line (see Table 6.3). The (dotted) vertical line represents the discrimination level between normal and abnormal performance on word fluency. WFS = word fluency score.

(B) Text reading performance and mean functional connectivity in the text reading fMRI paradigm. The (dotted) vertical line represents the discrimination level between normal and abnormal performance on text reading. No significant relation between reading score and functional connectivity was found for the entire patient group (see Table 6.3). TRS = text reading score.

\section{Clinical characteristics}

No correlation was observed between mean functional connectivity values of the word generation and text reading tasks and epilepsy characteristics, comprising number of partial and generalized seizures, age at onset of epilepsy, and drug load. Also, no effect of seizure focus could be demonstrated $(p>0.05)$. 
Table 6.3 Correlations of $\mathrm{fMRI}$ results with neuropsychological and clinical characteristics.

\begin{tabular}{|c|c|c|c|c|c|}
\hline & & $\begin{array}{l}\text { Functional } \\
\text { connectivity of word } \\
\text { generation }\end{array}$ & $p$-value & $\begin{array}{l}\text { Functional } \\
\text { connectivity of text } \\
\text { reading }\end{array}$ & $p$-value \\
\hline \multirow[t]{3}{*}{ Word fluency score } & Patients & $r_{P}=0.36$ & $0.04^{*}$ & $r_{P}=0.23$ & 0.18 \\
\hline & Patients with WFS $\leq 34$ & $Z_{c}=1.01 \pm 0.21$ & \multirow{2}{*}{$<0.01^{*}$} & $Z_{c}=0.72 \pm 0.217$ & \multirow{2}{*}{0.03} \\
\hline & Controls & $Z_{c}=1.27 \pm 0.16$ & & $\left.Z_{c}=0.88 \pm 0.22\right\rfloor$ & \\
\hline \multirow[t]{3}{*}{ Text reading score } & Patients & $r_{P}=0.44$ & $<0.01^{*}$ & $r_{P}=0.28$ & 0.12 \\
\hline & Patients withTRS $\leq 176$ & $Z_{c}=1.03 \pm 0.22$ & \multirow{2}{*}{$<0.01^{*}$} & $Z_{c}=0.68 \pm 0.17$ & \multirow{2}{*}{$<0.01^{*}$} \\
\hline & Controls & $Z_{c}=1.27 \pm 0.16$ & & $Z_{c}=0.88 \pm 0.22$ & \\
\hline Number of SGS & & $r_{S}=0.06$ & 0.74 & $r_{S}=-0.07$ & 0.70 \\
\hline \multirow{2}{*}{\multicolumn{2}{|c|}{$\begin{array}{l}\text { Number of partial } \\
\text { seizures }\end{array}$}} & & & & \\
\hline & & $r_{S}=-0.23$ & 0.20 & $r_{S}=0.22$ & 0.21 \\
\hline \multicolumn{2}{|l|}{ Age at onset } & $r_{p}=0.08$ & 0.64 & $r_{P}=-0.02$ & 0.90 \\
\hline \multicolumn{2}{|l|}{ Drug load } & $r_{P}=-0.24$ & 0.17 & $r_{P}=-0.06$ & 0.72 \\
\hline
\end{tabular}

Statistically significant after controlling for false discovery rate.

$\mathrm{Z}_{\mathrm{c}}=$ Fisher Z-value for functional connectivity expressed as mean $\pm \mathrm{SD} ; \mathrm{r}_{\mathrm{P}}=$ Pearson's correlation coefficient; $r_{S}=$ Spearman's correlation coefficient; WFS = word fluency score; TRS = text reading score; SGS = secondarily generalized seizures.

\section{Discussion}

For the first time, it was demonstrated that patients with cryptogenic localizationrelated epilepsy display difficulties in language functions, which relate to loss of functional connectivity in the language networks.

Several studies have addressed the relationship of language and epilepsy. Patients with a wide variety of epilepsy syndromes may display interictal language impairment. In a survey of language skills in 60 patients with different epilepsy syndromes, almost $30 \%$ of the patients demonstrated difficulties. ${ }^{33}$ Patients with left TLE exhibited poorer language performance than patients with right TLE, ${ }^{4}$ though it was suggested that both groups display language difficulties. ${ }^{34}$ Except for an early onset of epilepsy, ${ }^{35}$ no other epilepsy parameter has been associated with poorer language performance. Our findings confirm the results from these previous studies demonstrating interictal language difficulties, but without an association with epilepsy characteristics.

Notable differences were observed in the functional connectivity between the involved brain regions, which indicates a reduced synchronization of activity in the language network. This impairment of language circuits manifested both in prefrontal and temporal regions. These findings illustrate that impaired language function may not necessarily be reflected by altered patterns or levels of cerebral activation, but may be characterized by improperly orchestrated activity in the language network. 
The reduction in functional connectivity in the word generation paradigm correlated with lower word fluency performance and reading scores in the epilepsy group. For word generation, the largest difference in functional connectivity between epilepsy patients and controls was found for the connection between the ACC and left-IFG region. This difference was most pronounced for the patients with lowest word fluency scores. This implies a relationship between loss of functional connectivity within cerebral circuits and deficient performance. For the $\mathrm{fMRI}$ reading paradigm, no direct correlation between a lower mean functional connectivity value and reading score or word generation performance could be demonstrated. However, functional connectivity differed significantly between patients and healthy controls, and this effect was even more notable when comparing those patients with worse reading scores to healthy controls. The largest difference in functional connectivity between epilepsy patients and controls was found between the MTG and IFG region in the left hemisphere. The observed differences between the word generation and text reading paradigms may have different explanations. First, the specificity of the applied fMRI paradigms to activate the different regions involved in the language network is probably higher for the word generation task than for the text reading task. Second, temporal brain areas are more prone to suffer from loss of BOLD-signal due to technical issues. ${ }^{36}$ With higher levels of activation, possible differences between patients and healthy controls are more easily detected. This might also explain why the results of the word generation paradigm are correlated with both neuropsychological tests and the text reading paradigm to neither. A third explanation is the involvement of the prefrontal cortex, which is more strongly involved in expressive word generation than in receptive reading. Apparently, the prefrontal cortex and its inherent high number of connections seem more prone to impairment than the temporal cortex in chronic epilepsy, but this suggestion needs independent proof.

Although the current study shows that decline of language function is associated with decreased network connectivity, one cannot exclusively ascribe the observed network changes to impaired language function as other high-order cognitive functions (e.g., attention processes and working memory) may be of influence and have a partly overlapping distribution of active brain areas and connections.

Recently, functional connectivity analysis has been performed in presurgical patients with left TLE. ${ }^{30}$ This study showed a reduced functional connectivity for the language areas in the resting state. The authors suggested a disturbance of the language network; a relation with neuropsychological performance was not investigated. Moreover, patients with left TLE showed an alteration of activation lateralization compared to controls. The current study differs in several aspects, since no differences in activation patterns were demonstrated in 2 language tasks, patients had no symptomatic lesions, and data from the activated state were used to calculate 
functional connectivity emphasizing the connectivity of the activated cortex. The current research extends on this by demonstrating that reduced language connectivity in epilepsy is a pathologic phenomenon that is not dependent on the presence of (temporal) lesions.

Whether the decrease in functional connectivity within the prefrontal network in our patients is associated with a reduction of structural connectivity as well was not investigated in this study. In previous research, resting state functional connectivity in healthy subjects reflected structural connectivity as measured with DTI. ${ }^{37}$ In studies with patients with TLE, altered functional language lateralization was reflected by a structural reorganization of white matter tracts ${ }^{38}$ and stronger white matter tract connections in the language-dominant hemisphere were associated with better naming scores. ${ }^{39}$ In another study with patients with TLE, an association of structural damage of multiple fiber tracts, especially in the left hemisphere, and language impairment was demonstrated as well. ${ }^{14}$

Although the differences in functional connectivity between patients with epilepsy and healthy controls in this study are evident, the underlying mechanisms remain to be elucidated. It is attractive to attribute all differences to whether the participant has epilepsy or not. It can be argued that the 2 groups differ in handedness, and thereby possibly in cerebral language organization. In a post-hoc analysis, however, no significant influence of handedness on our results could be demonstrated. It could nonetheless be interesting to study larger groups of left-handed patients and healthy controls to assess possible differences. Furthermore, it would be interesting to compare our results with those of patients with dyslexia. Changes in cerebral network connectivity, though different from those in our study, have recently been demonstrated. ${ }^{40}$ If our results are maintained even after comparison with other language-impaired patients without evident structural brain damage, the influence of epilepsy can be confirmed.

Further studies are required to assess the individual conditions leading to altered functional connectivity in patients, which would ideally lead to the identification of patients at risk for developing cognitive impairment, issues regarding causality, and the improvement of therapeutic decisions. 


\section{References}

1. Oyegbile TO, Dow C, Jones J, Bell B, Rutecki P, Sheth R, Seidenberg M, Hermann BP. The nature and course of neuropsychological morbidity in chronic temporal lobe epilepsy. Neurology. 2004;62: 1736-42.

2. Mayeux R, Brandt J, Rosen J, Benson DF. Interictal memory and language impairment in temporal lobe epilepsy. Neurology. 1980;30:120-5.

3. Hermann BP, Wyler AR, Steenman H, Richey ET. The interrelationship between language function and verbal learning/memory performance in patients with complex partial seizures. Cortex. 1988;24: 245-53.

4. Hermann BP, Seidenberg M, Haltiner A, Wyler AR. Adequacy of language function and verbal memory performance in unilateral temporal lobe epilepsy. Cortex. 1992;28:423-33.

5. Helmstaedter C, Elger CE. Behavioral markers for self- and other-attribution of memory: a study in patients with temporal lobe epilepsy and healthy volunteers. Epilepsy Res. 2000;41:235-43.

6. Jokeit H, Kramer G, Ebner A. Do antiepileptic drugs accelerate forgetting? Epilepsy Behav. 2005;6: 430-2.

7. Thompson PJ, Duncan JS. Cognitive decline in severe intractable epilepsy. Epilepsia. 2005;46:1780-7.

8. Aldenkamp A, Arends J. The relative influence of epileptic EEG discharges, short nonconvulsive seizures, and type of epilepsy on cognitive function. Epilepsia. 2004;45:54-63.

9. Dodrill CB, Batzel LW. Interictal behavioral features of patients with epilepsy. Epilepsia. 1986;27 Suppl 2:S64-76.

10. Oyegbile TO, Bhattacharya A, Seidenberg M, Hermann BP. Quantitative MRI biomarkers of cognitive morbidity in temporal lobe epilepsy. Epilepsia. 2006;47:143-52.

11. Hermann BP, Seidenberg M, Dow C, Jones J, Rutecki P, Bhattacharya A, Bell B. Cognitive prognosis in chronic temporal lobe epilepsy. Ann Neurol. 2006;60:80-7.

12. Seidenberg M, Geary E, Hermann B. Investigating temporal lobe contribution to confrontation naming using MRI quantitative volumetrics. J Int Neuropsychol Soc. 2005;11:358-66.

13. Hermann B, Hansen R, Seidenberg M, Magnotta V, O'Leary D. Neurodevelopmental vulnerability of the corpus callosum to childhood onset localization-related epilepsy. Neuroimage. 2003;18:284-92.

14. McDonald CR, Ahmadi ME, Hagler DJ, Tecoma ES, Iragui VJ, Gharapetian L, Dale AM, Halgren E. Diffusion tensor imaging correlates of memory and language impairments in temporal lobe epilepsy. Neurology. 2008;71:1869-76.

15. Cole AJ. Is epilepsy a progressive disease? The neurobiological consequences of epilepsy. Epilepsia. 2000;41 Suppl 2:S13-22.

16. Fuerst D, Shah J, Kupsky WJ, Johnson R, Shah A, Hayman-Abello B, Ergh T, Poore Q, Canady A, Watson C. Volumetric MRI, pathological, and neuropsychological progression in hippocampal sclerosis. Neurology. 2001;57:184-8.

17. Berl MM, Balsamo LM, Xu B, Moore EN, Weinstein SL, Conry JA, Pearl PL, Sachs BC, Grandin CB, Frattali C, Ritter FJ, Sato $S$, Theodore WH, Gaillard WD. Seizure focus affects regional language networks assessed by fMRI. Neurology. 2005;65:1604-11.

18. Gaillard WD, Berl MM, Moore EN, Ritzl EK, Rosenberger LR, Weinstein SL, Conry JA, Pearl PL, Ritter FF, Sato S, Vezina LG, Vaidya CJ, Wiggs E, Fratalli C, Risse G, Ratner NB, Gioia G, Theodore WH. Atypical language in lesional and nonlesional complex partial epilepsy. Neurology. 2007;69:1761-71.

19. Mbwana J, Berl MM, Ritzl EK, Rosenberger L, Mayo J, Weinstein S, Conry JA, Pearl PL, Shamim S, Moore EN, Sato S, Vezina LG, Theodore WH, Gaillard WD. Limitations to plasticity of language network reorganization in localization related epilepsy. Brain. 2009;132:347-56.

20. Duncan J, Owen AM. Common regions of the human frontal lobe recruited by diverse cognitive demands. Trends Neurosci. 2000;23:475-83.

21. Swanson SJ, Sabsevitz DS, Hammeke TA, Binder JR. Functional magnetic resonance imaging of language in epilepsy. Neuropsychol Rev. 2007;17:491-504.

22. Lammers MW, Hekster YA, Keyser A, Meinardi H, Renier WO, van Lier H. Monotherapy or polytherapy for epilepsy revisited: a quantitative assessment. Epilepsia. 1995;36:440-6.

23. Wechsler D. Manual for the Wechsler Adult Intelligence Scale-III. San Antonio: The Psychological Corporation; 1997. 
24. Annett M. A classification of hand preference by association analysis. Br J Psychol. 1970;61:303-21.

25. Deelman BG, Liebrand $W B$, Koning-Haanstra $M$, van den Burg W. [Measurements of aphasic disorders. A brief description of the SAN-battery]. Gerontologie. 1980;11:17-21.

26. Deblaere K, Backes WH, Hofman P, Vandemaele P, Boon PA, Vonck K, Boon P, Troost J, Vermeulen J, Wilmink J, Achten E, Aldenkamp A. Developing a comprehensive presurgical functional MRI protocol for patients with intractable temporal lobe epilepsy: a pilot study. Neuroradiology. 2002;44:667-73.

27. Backes WH, Deblaere K, Vonck K, Kessels AG, Boon P, Hofman P, Wilmink JT, Vingerhoets G, Boon PA, Achten R, Vermeulen J, Aldenkamp AP. Language activation distributions revealed by fMRI in postoperative epilepsy patients: differences between left- and right-sided resections. Epilepsy research. 2005;66:1-12.

28. Nichols TE, Holmes AP. Nonparametric permutation tests for functional neuroimaging: a primer with examples. Hum Brain Mapp. 2002;15:1-25.

29. Riecker A, Grodd W, Klose U, Schulz JB, Groschel K, Erb M, Ackermann H, Kastrup A. Relation between regional functional MRI activation and vascular reactivity to carbon dioxide during normal aging. J Cereb Blood Flow Metab. 2003;23:565-73.

30. Waites AB, Briellmann RS, Saling MM, Abbott DF, Jackson GD. Functional connectivity networks are disrupted in left temporal lobe epilepsy. Ann Neurol. 2006;59:335-43.

31. Zar JH. Biostatistical analysis. 3rd ed. Upper Saddle River: Prentice-Hall; 1996:376.

32. Benjamini $Y$, Hochberg Y. Controlling the false discovery rate - a practical and powerful approach to multiple testing. Journal of the Royal Statistical Society Series B-Methodological. 1995;57:289-300.

33. Davey D, Thompson P. Interictal language functioning in chronic epilepsy. J Neurolinguistics. 1991;6:381-99.

34. Hermann BP, Bell B, Seidenberg M, Woodard A. Learning disabilities and language function in epilepsy. Epilepsia. 2001;42 Suppl 1:21-3; discussion 8.

35. Schoenfeld J, Seidenberg M, Woodard A, Hecox K, Inglese C, Mack K, Hermann B. Neuropsychological and behavioral status of children with complex partial seizures. Dev Med Child Neurol. 1999;41: 724-31.

36. Powell HW, Koepp MJ, Richardson MP, Symms MR, Thompson PJ, Duncan JS. The application of functional MRI of memory in temporal lobe epilepsy: a clinical review. Epilepsia. 2004;45:855-63.

37. Greicius MD, Supekar K, Menon V, Dougherty RF. Resting-state functional connectivity reflects structural connectivity in the default mode network. Cereb Cortex. 2009;19:72-8.

38. Powell HW, Parker GJ, Alexander DC, Symms MR, Boulby PA, Wheeler-Kingshott CA, Barker GJ, Koepp MJ, Duncan JS. Abnormalities of language networks in temporal lobe epilepsy. Neuroimage. 2007;36:209-21.

39. Powell HW, Parker GJ, Alexander DC, Symms MR, Boulby PA, Barker GJ, Thompson PJ, Koepp MJ, Duncan JS. Imaging language pathways predicts postoperative naming deficits. J Neurol Neurosurg Psychiatry. 2008;79:327-30.

40. Wolf RC, Sambataro F, Lohr C, Steinbrink C, Martin C, Vasic N. Functional brain network abnormalities during verbal working memory performance in adolescents and young adults with dyslexia. Neuropsychologia. 2010;48:309-18. 


\section{Chapter}

Loss of network efficiency associated with cognitive decline in chronic epilepsy

MCG Vlooswijk*, MJ Vaessen*, JFA Jansen, MCFTM de Krom, HJM Majoie, PAM Hofman, AP Aldenkamp, WH Backes

*both authors contributed equally

Neurology, 2011;77:938-944 


\section{Abstract}

\section{Objective}

To study the relation between possibly altered whole brain topology and intellectual decline in chronic epilepsy, a combined study of neurocognitive assessment, and fMRI with graph theoretical network analysis was performed.

\section{Methods}

Forty-one adult patients with cryptogenic localization-related epilepsy and 23 healthy controls underwent an intelligence test and $\mathrm{FMRI}$ with a silent word generation paradigm. A set of undirected graphs was constructed by cross-correlating the signal time-series of 893 cortical and subcortical regions. Possible changes in cerebral network efficiency were assessed by performing graph theoretical network analysis.

\section{Results}

Healthy subjects displayed efficient small world properties, characterized by high clustering and short path lengths. On the contrary, in patients with epilepsy a disruption of both local segregation and global integration was found. An association of more pronounced intellectual decline with more disturbed local segregation was observed in the patient group. The effect of antiepileptic drug use on cognitive decline was mediated by decreased clustering.

\section{Conclusions}

These findings support the hypothesis that chronic localization-related epilepsy causes cognitive deficits by inducing global cerebral network changes instead of a localized disruption only. Whether this is the result of epilepsy per se or the use of antiepileptic drugs remains to be elucidated. For application in clinical practice, future studies should address the relevance of altered cerebral network topology in prediction of cognitive deficits and monitoring of therapeutic interventions. 


\section{Introduction}

In chronic epilepsy, patients often experience cognitive problems ${ }^{1}$ extending from memory deficits ${ }^{2}$ to language problems ${ }^{3}$ and intellectual impairment. ${ }^{4}$ Clinical factors such as antiepileptic drugs (AED), ${ }^{5}$ and high seizure frequency ${ }^{6}$ cannot always predict the individual cognitive course.

To understand the neurobiological mechanisms of cognitive dysfunction in localization-related epilepsy, functional MRI (fMRI) research has focused on changes in activation patterns. Most $\mathrm{fMRI}$ studies report an association of cognitive dysfunction with either decreased activation ${ }^{7-9}$ or a shift of activation. ${ }^{10-12}$

The focus of fMRI research in epilepsy and cognition has changed to analyzing dysfunctional networks. Most studies measure functional connectivity by correlating signal time-courses of different cerebral regions. ${ }^{13}$ Typically, higher functional connectivity is associated with better cognitive performance. ${ }^{14-16}$

With conventional functional connectivity methods applying a priori selection of specific networks unexpected abnormalities outside these networks can remain undetected. With graph theoretical analysis the organization of the whole brain network can be investigated (for a review, see ${ }^{17}$ ). A distinction can be made between a 'small-world' and a random topology. Most studies ${ }^{18}$ demonstrate that brain networks are organized as 'small-world' networks which are more efficient than 'random' networks. ${ }^{19}$

We aimed to investigate the changes in functional networks using graph theoretical network analysis in patients with epilepsy in relation to intellectual performance and possible decline. We hypothesize that: i. patients will have lower intellectual performance; ii. patients will have a less efficient organized network than healthy controls; and iii. network abnormalities will be more pronounced in those patients with lowest IQ and distinct intellectual decline.

\section{Methods}

\section{Participants}

Inclusion criteria for the patients were: localization-related epilepsy with an epileptic focus in the frontal or temporal lobe, absence of structural cerebral lesions other than mesiotemporal sclerosis (MTS), no history of status epilepticus and no other disease that could cause cognitive decline. The final study population included 41 patients 
( 21 women; mean age 40 years; range $22-63$ ) and 23 healthy controls (14 women; mean age 40 years; range 18-58). See Table 7.1 for an overview of the clinical characteristics of the patients and healthy controls.

\section{Standard protocol approvals, registrations, and patient consents}

This study was approved by the Institutional Review Board of the Maastricht University Medical Center. All subjects gave written informed consent.

\section{Neuropsychological testing}

For intelligence, the third Wechsler Adult Intelligence Scale (WAIS-III) was used. ${ }^{20}$ An estimate of premorbid intelligence levels was made according to the formula proposed by Schoenberg et al. ${ }^{21}$ Intelligence discrepancy scores were calculated by subtracting premorbid full-scale IQ (FSIQ) estimates from actual FSIQ, resulting in IQ discrepancy scores (see Appendix 7.1 for details).

\section{MRI protocol}

All subjects underwent a clinical epilepsy protocol on a 3.0 Tesla unit with an 8channel head coil. Functional MRI data were acquired using echo-planar imaging pulse sequence (repetition time (TR) $2 \mathrm{~s}$, echo time (TE) $5 \mathrm{~ms}$, flip angle $90^{\circ}$, voxel size $2 \times 2 \times 4 \mathrm{~mm})$ and 196 volumes per acquisition. For anatomic reference, a T1-weighted three-dimensional (3D) fast gradient echo was acquired (TR $9.91 \mathrm{~ms}$, TE $4.6 \mathrm{~ms}$, inversion time (TI) $3 \mathrm{~s}$, flip angle $8^{\circ}$, voxel size $1 \times 1 \times 1 \mathrm{~mm}$ ).

\section{fMRI activation paradigm}

In the word generation paradigm, subjects had to covertly generate as many words as possible starting with a visually presented letter (U-N-K-A-E-P). The paradigm consisted of six word generation condition blocks (one letter per $30 \mathrm{~s}$ block) alternated with baseline rest condition blocks (30 s). Afterwards, all subjects were able to sufficiently reproduce words generated during the task.

\section{Image analysis}

\section{Image preprocessing}

Analysis of the time-series data was performed in the Statistical Parametric Mapping (SPM2) software application (Wellcome Department of Cognitive Neurology, London, UK). Dynamic images were slice-timed and realigned to correct for head movement. The corrected images were transformed into standard MNI space and spatially smoothed (6-mm kernel). 


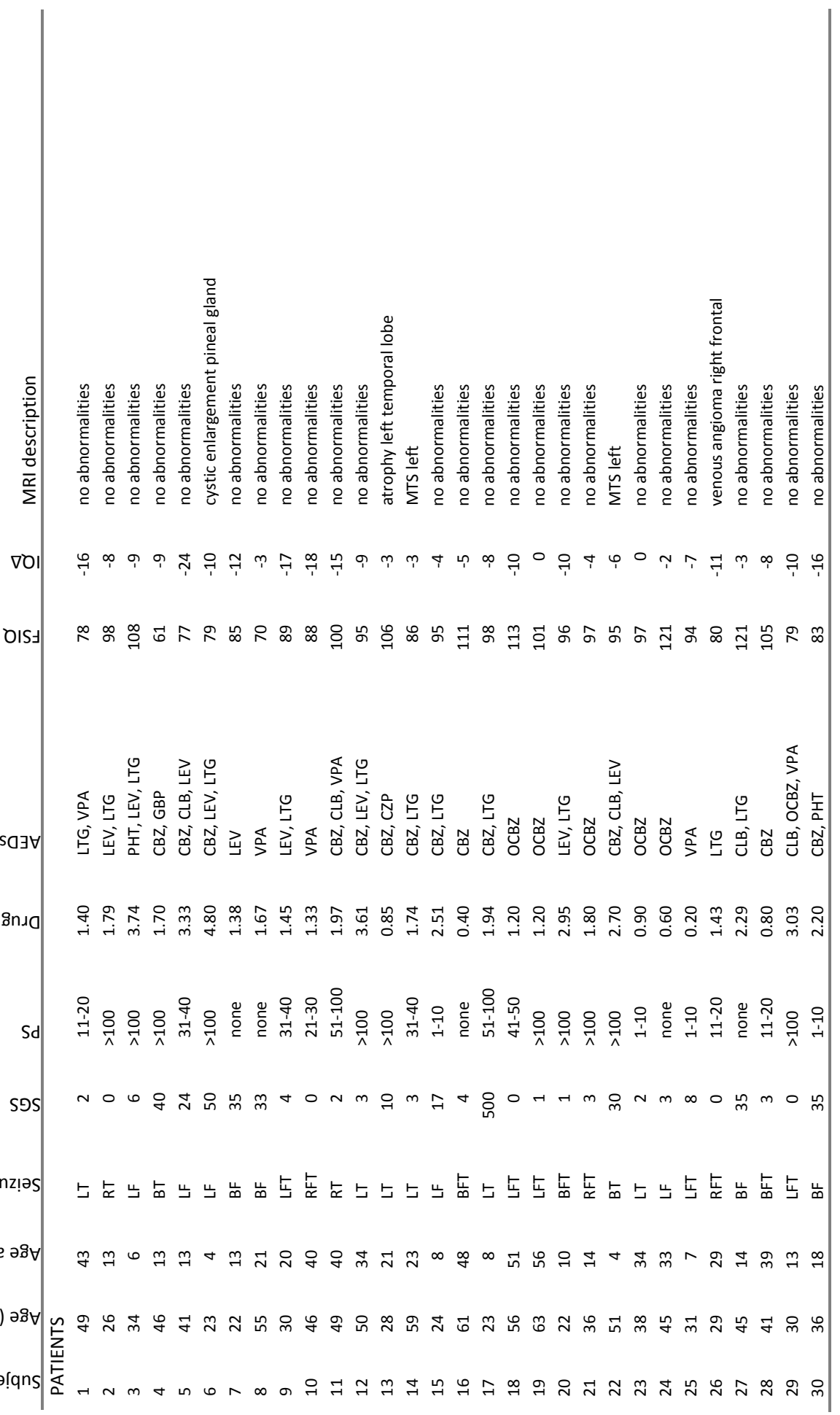


118 Chapter 7

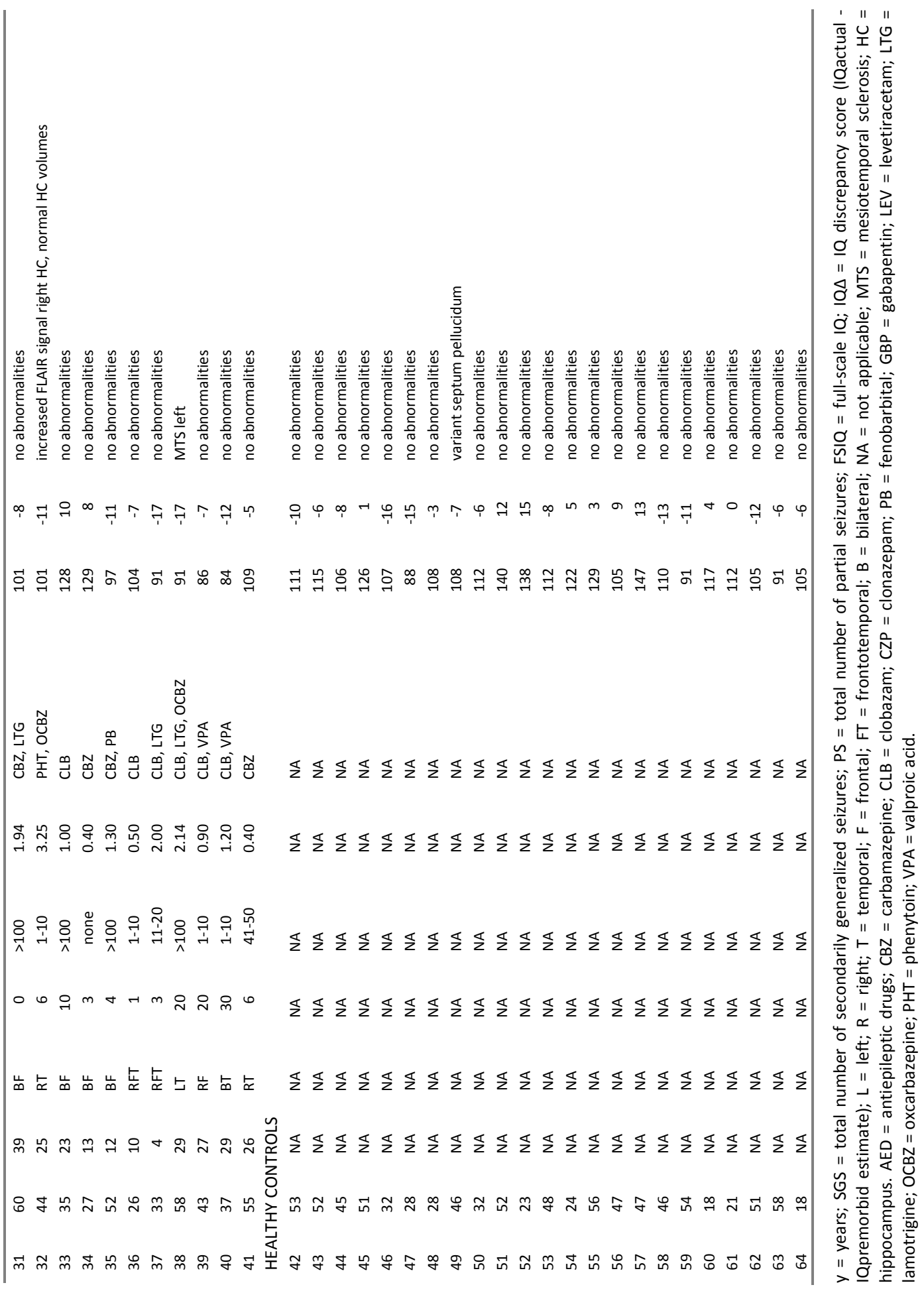




\section{Whole brain network construction}

The preprocessed and normalized $\mathrm{fMRI}$ images were parcellated into a high resolution network consisting of $N=893$ cortical and subcortical brain regions (see Appendix 7.2 for details on the parcellation scheme). Characteristic time-series were calculated by averaging the signal intensities from all voxels in a region. To reduce the effect physiological noise and movement related noise, ${ }^{22}$ the time-series were filtered by applying standard linear regression with the movement parameters as a co-variate and by applying a band pass filter $(0.01-0.1 \mathrm{~Hz})$.

\section{Network parameters}

Graph theoretical parameters were used to evaluate the functional networks. ${ }^{17,23,24} \mathrm{~A}$ connection matrix $A$ was formed by calculating Pearson correlation coefficients between all pairs of brain regions. In the brain graph, a node is related to a brain region, an edge is a connection between brain areas $i$ and $j$.

The brain graph of each individual was thresholded to create graphs with an equal number of nodes and edges across subjects. ${ }^{25}$ This was achieved by selecting the $T_{k}$ connections with the highest correlation coefficient and removing all other connections. The threshold value $T_{k}$ was expressed as a sparsity value relating the number of edges maintained in the network to the total number of edges possible $\left(N^{2}-N\right) .{ }^{26}$ Let $T_{k}$ be the number of edges maintained in the network, then the sparsity $(s)$ is defined as:

$$
\text { Sparsity }=\left(N^{2}-N-T_{k}\right) /\left(N^{2}-N\right)
$$

As there is no theoretical criterion for which sparsity value is the most biologically meaningful, here we explored network parameters over a range of sparsity values. To guarantee high correlation coefficients of the remaining connections, the sparsity range was chosen to be higher than 0.87 , which yielded an average correlation coefficient of 0.66 .

The graph theoretical metrics characteristic path length $(L)$ and cluster coefficient $(C)$ as well as local and global efficiency were calculated to perform analysis on the constructed brain graphs. The characteristic path length is a measure of how well connected a network is. The cluster coefficient of a network is a measure of how many local clusters exist in the network. Parameters related to characteristic path length are global efficiency $\left(E_{\text {global }}\right)$ and local efficency $\left(E_{\text {local }}\right)$. $E_{\text {global }}$ is defined as the average inverse shortest path length, $E_{\text {local }}$ is defined as the mean of the global efficiencies of subgraphs consisting of the immediate neighbours of a particular node. $^{26}$ To be able to determine whether a network has small-world properties, the 
values of $L$ and $C$ must be scaled to values from generated random networks. ${ }^{24}$ Small world networks are characterized by having $L$ close to random: $\lambda=L / L_{\text {random }} \approx 1$, but with $C$ higher than random: $y=C / C_{\text {random }}>1$. See Appendix 7.2 for a more elaborate description of these metrics.

\section{Statistical analysis}

Group differences of graph theoretical network parameters were assessed with the Student's $t$-test. The association between clinical variables (age, age at onset and drug load), cognitive variables (FS-IQ and IQ discrepancy) and network parameters were analyzed using Pearson's correlation coefficients. The relation between clinical seizure variables and graph theoretical parameters with intellectual decline was examined using a mediator-model approach. ${ }^{27}$ With this model, it is investigated whether network parameters mediate the relation between clinical and cognitive characteristics. Analyses were performed with graph theoretical network parameters and drug load (the clinical variable with the strongest link to cognition in this dataset) as independent and intellectual decline as the dependent variable. If the relation between drug load and intellectual decline becomes non-significant when network parameters are entered in the model, this parameter can be considered a mediator of the relation between drug load and intellectual decline.

\section{Results}

\section{Neuropsychological assessment}

Patients had lower FSIQ than controls (96 \pm 15 vs. $113 \pm 15, p<0.01$ ). In the patient group, IQ discrepancy scores were significantly lower than in the controls, indicating intellectual decline in the patient group as a whole (mean \pm standard deviation IQ discrepancy score $-8.6 \pm 6.5$ in patients vs $-3.6 \pm 8.8$ in controls, $p=0.02$ ). For the individual patients, seven had lower IQ discrepancy scores than the minimum score in the control group; they had evident intellectual decline (impaired group).

\section{Functional MRI results}

\section{Activation map results}

Activation maps of the word generation paradigm revealed significantly activated clusters in the left inferior and left middle frontal cortex (Broca region), the right middle frontal cortex, and the anterior cingulate cortex for both groups. No significant differences were found between controls and patients with epilepsy (see ${ }^{3}$, for details). 


\section{fMRI graph theoretical network parameters}

Both patient and control networks showed a topology in the small-world regime with values for $\lambda$ close to 1 and values for $y$ higher than 1 . Patients displayed significantly lower values $(p<0.05)$ for $\gamma, E_{\text {global }}$ and $E_{\text {local }}$ over almost the entire sparsity range (Figure 7.1). A trend towards higher $\lambda$ values was observed in the patient group, which was significantly higher for high sparsity values $(s=0.96-0.97)$. For $C$, patients also had lower values for the highest sparsity values. The network parameters of the impaired group revealed significant lower values for $C$ over a broader sparsity range as compared with unimpaired patients and controls. Impaired patients displayed significantly higher values than controls for $L(s=0.94-0.95)$ and $\lambda(s=0.94-0.96)$ (Figure 7.2).

Summarizing, all the encountered differences point toward a disruption of network integrity characterized by a more random network topology in the patient group.
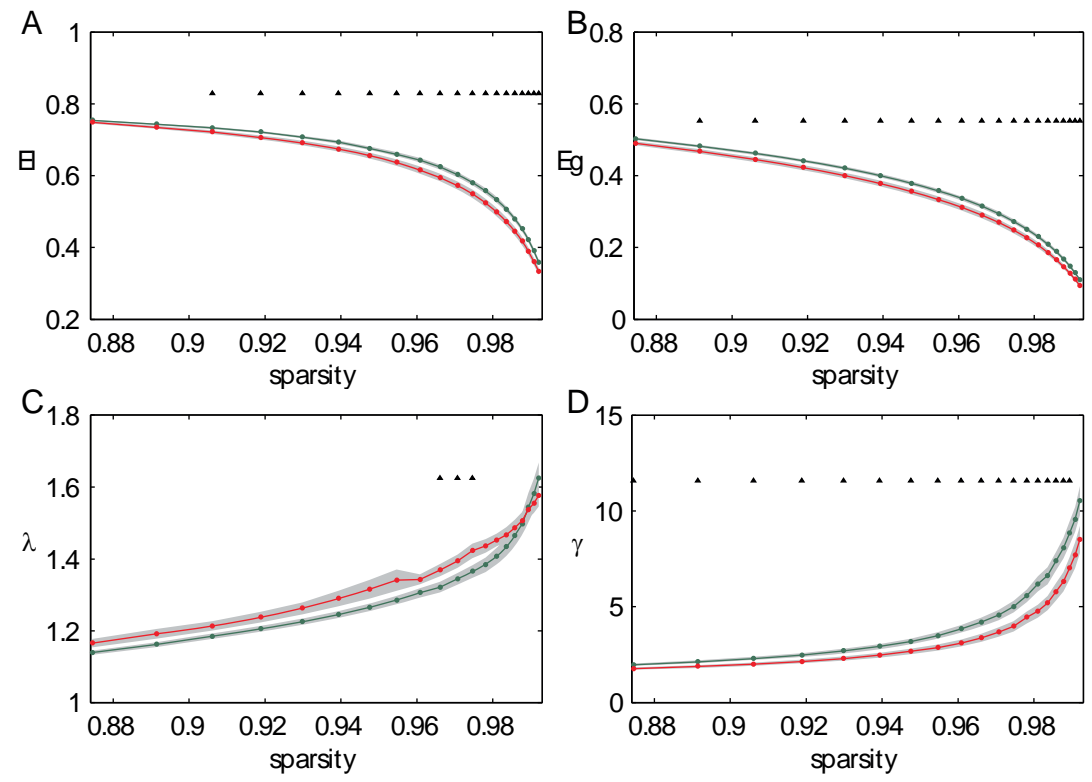

Figure 7.1 Network parameters for the whole brain networks of the patient group (red lines) and the control group (green lines) as function of sparsity. Black triangles indicate for which sparsity values the groups differed significantly, demonstrating consistently lower values for $E_{\text {global, }}$, $E_{\text {local }}$ and $\gamma$ and higher values for $\lambda$ in the patient group. Grey areas indicate standard error of the mean. (A) Local efficiency, (B) global efficiency, (C) $\lambda$, and (D) $\gamma$. 
A

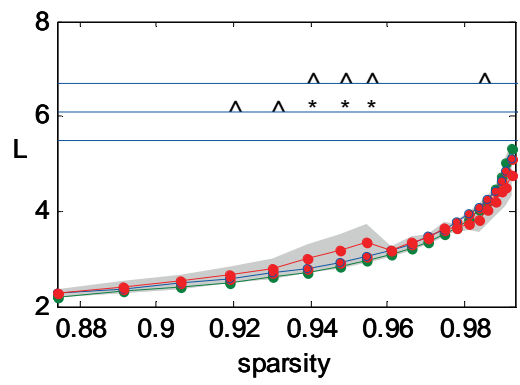

B

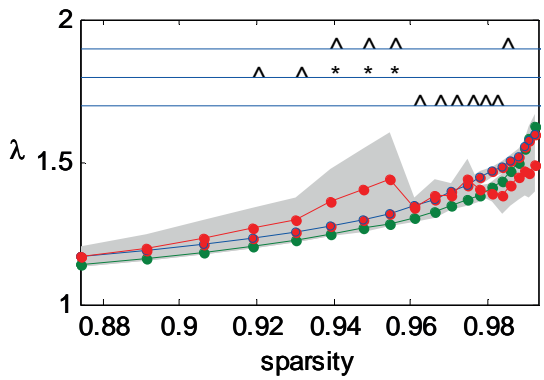

Figure 7.2 Path length and lambda parameters for the whole brain network including cognitively impaired patients. Networks of the impaired patient group (red lines), the unimpaired patient group (blue lines) and the control group (green lines) as function of sparsity. (A) Path length $(L)$, (B) lambda $(\lambda)$. Grey areas indicate standard error of the mean for each of the analyzed groups.

* Single asterisks indicate for which sparsity values the impaired group showed a statistical trend $(p<0.1)$ difference from the unimpaired group (see line with ' $(i-u)$ ' in the figure), demonstrating higher values for lambda in the impaired patient group.

** Double asterisks indicate for which sparsity values the impaired group showed a statistical trend $(p<0.1)$ difference from the control and double triangles indicate for which sparsity values the impaired group differed significantly $(p<0.05)$ from the control group, (see line with '( $i-c)$ ' in the figure). This shows higher path length and lower lambda (normalized path lengths) for the impaired patient group.

*** Triple asterisks indicate for which sparsity values the unimpaired group showed a statistical trend $(p<0.1)$ difference from the control, see line with ' $(u-c)$ ' in the figure. Here, the unimpaired patient group displays higher path lengths and lower lambdas compared to the control group.

\section{Regional analysis of network parameters}

Additional analysis was carried out to investigate whether the majority of identified network abnormalities are localized in certain brain regions or networks. Results are only shown for $\gamma$, since $\gamma$ was the network parameter with the most pronounced difference between patients and controls. As can be observed from Figure 7.3, a number of regions did show significant differences $(p<0.005)$. However, these regions were evenly distributed throughout the whole brain, without evident grouping within a specific lobe or recognizable network. When controls were compared to the impaired patients only, similar results were obtained. No associations of side of seizure focus with distribution of affected regions were observed. 

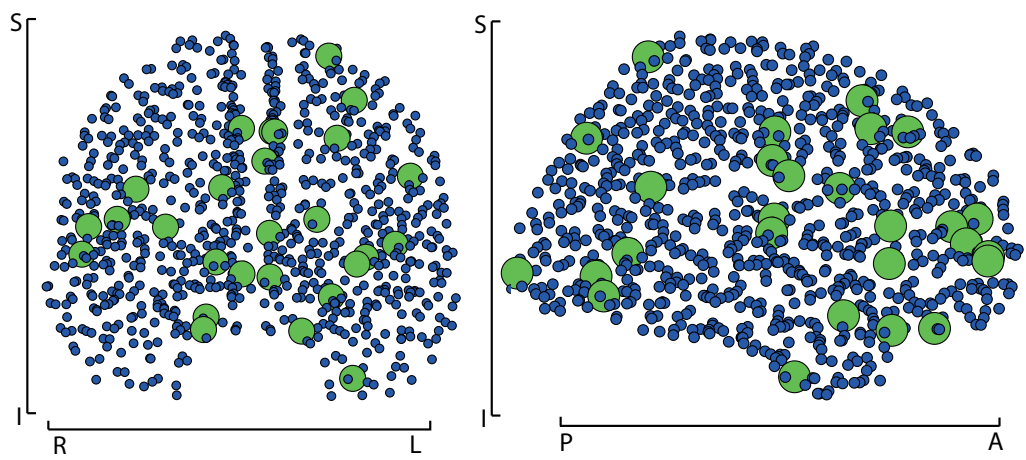

Figure 7.3 3D projection of the network nodes. Green circles indicate where the nodal values of $\gamma$ were significantly lower in the patient group $(p<0.005)$. The affected nodes are distributed throughout the whole brain, not restricted to specific lobes or functional networks. $\mathrm{S}=$ superior; $\mathrm{I}=$ inferior; $\mathrm{L}=$ left; $\mathrm{R}=$ right; $\mathrm{A}=$ anterior; $\mathrm{P}=$ posterior.

\section{fMRI graph theoretical network parameters in correlation with neuropsychological parameters}

The cluster coefficient was found to be positively associated with FSIQ over a range of sparsity values $(s=0.87-0.97)$. This same effect was seen for the association between $C$ and IQ discrepancy $(s=0.93-0.98)$. Hence, a decreased amount of clustering in the activated patient brain indicates a reduced IQ, and a decreased clustering relates to a more pronounced intellectual decline. For the other network parameters, no correlation with FSIQ and IQ discrepancy was demonstrated. In the control group, there was no association between any network parameter and FSIQ.

\section{Correlation of fMRI graph theoretical network parameters with clinical factors}

From the clinical factors available, drug load was negatively associated with $C, y$ and $E_{\text {local, }}$ indicating a more random and less efficient network with higher drug load (for $\mathrm{C}$ : $p<0.03$ for sparsity range $0.97-0.99$; for $\gamma$ : $p$-values range from 0.03 to 0.06 in the entire sparsity range; for $\mathrm{E}_{\text {local }}: p<0.05$ for sparsity range $\left.0.87-0.93\right)$. For the other clinical characteristics (age and age at onset), no significant associations were found.

\section{Model for the relation between clinical characteristics, network parameters and intellectual decline}

The relation of clinical epilepsy variables and graph theoretical network parameters to intellectual decline was examined using the mediator analysis. Since drug load had the 
strongest correlation with intellectual decline $(p=0.03)$, this factor was entered in the mediator analysis. The other clinical characteristics did not meet the criteria to be entered in a mediator analysis. ${ }^{27}$ Drug load was no longer a significant predictor of intellectual decline when the clustering coefficient $C$ was included in the model (Figure 7.4). On the contrary, $C$ was the mediating factor between drug load and intellectual decline, indicating that abnormalities in graph theoretical network parameters, particularly the clustering coefficient $C$, mediated the impact of drug load on cognition ( $s=0.96-0.98)$.
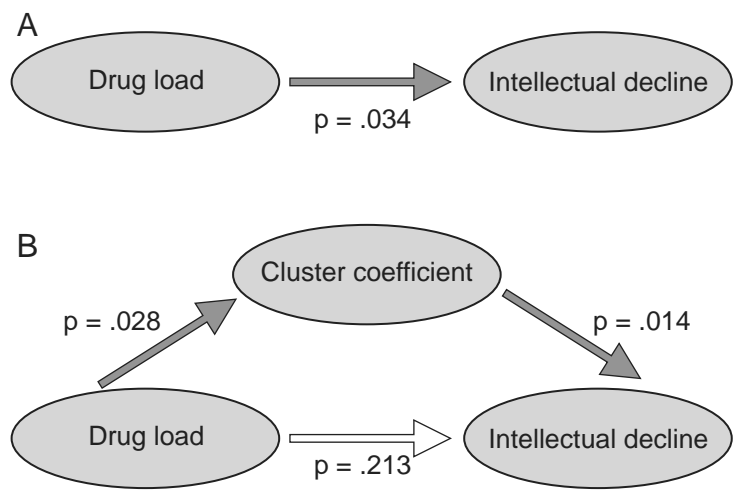

Figure 7.4 The mediator analysis. A. Drug load as a predictor of intellectual decline when graph theoretical network parameters are not considered. B. When graph theoretical network parameters are considered, the cluster coefficient $(C)$ mediates the relation between drug load and intellectual decline. $P$-values represent the values for sparsity value 0.97 .

\section{Discussion}

\section{Current findings}

In the present study, fMRI time-series data from a language paradigm were used to evaluate functional brain networks in patients with chronic epilepsy and healthy controls. Graph theoretical network parameters were compared between patients and controls and compared with cognitive performance. First, the patients with epilepsy displayed disturbed network parameters, such as a lower normalized clustering coefficient $(\gamma)$, and lower local and global efficiencies. Second, for the subgroup of patients with most pronounced cognitive decline, lower absolute clustering coefficient, and higher absolute and normalized path length were observed. Third, cognitive status (IQ) and the degree of intellectual decline (IQ discrepancy score) were correlated with graph theoretical network parameters, which revealed 
that poorer cognitive status and more pronounced intellectual decline were associated with lower absolute clustering coefficients. This is the first study that describes a relation between disturbed graph theoretical network parameters and cognitive dysfunction in patients with chronic epilepsy. Fourth, as expected, ${ }^{19,28}$ the topological parameters were consistent with a small-world organization of the cerebral networks in the control group.

\section{Previous findings}

Lower clustering coefficients ${ }^{28}$ and an increased path length ${ }^{25,28}$ have been demonstrated in neuropsychiatric disorders such as Alzheimer's disease and schizophrenia. These disorders are accompanied with pronounced cognitive deficits, although graph theoretical network parameters have not previously been linked directly with cognitive measures.

Previously, in patients with bilateral mesial temporal lobe epilepsy whole-brain graph network analysis demonstrated lower absolute and normalized path lengths together with a decrease in absolute clustering coefficients. ${ }^{29}$ This was interpreted as a disruption of the whole brain network with a more random topology, which is comparable to our results. The apparent contradictive results concerning path length are difficult to explain due to differences in study population and methodology. For example, patients in the latter study were on average much younger which might have influenced the results. One might hypothesize that brain networks of younger patients respond differently to disease. For instance, young patients might be able to compensate better by acquiring alternate brain regions for cognitive processing, while older patients might have lost this ability. This could lead to a different expression of networks parameters between control and patient groups in different age categories.

\section{Network analysis}

Cognitive functioning depends on several cerebral networks instead of isolated brain regions. It is reasonable to assume that in patients with epilepsy a disruption of whole brain networks is involved in the development of cognitive deficits, instead of a localized disruption at the site of seizure focus only. This is supported by MR studies demonstrating volumetric loss, ${ }^{30,31}$ microstructural white matter abnormalities, ${ }^{32}$ cortical thinning ${ }^{33}$ and functional abnormalities ${ }^{3,15}$ outside the epileptic focus. The application of methods focusing on localized abnormalities seems to be limited in patient populations with heterogeneous seizure foci and cognitive deficits. In our study population, this is supported by the fact that no localized disruptions in the task activation maps were found in the patient group compared to the controls. The silent word generation task does not allow for objective assessment of task performance. Theoretically, differences in task performance could affect the network parameters. 
Nevertheless, based on post-task performance assessment and careful inspection of individual activation maps it is unlikely that salient differences on task performance have influenced the fMRI data. Graph theoretical network analysis allows for analysis of whole brain networks rather than at a local level. Hence, it might be better capable of detecting patient specific abnormalities in functional brain organization and reorganization than more conventional (single connection) analysis methods. Indeed, the disruption of small-world characteristics in our patient group could not be localized within one or more specialized brain regions, which could represent diffuse disruptions throughout the whole brain. No global technical differences between patients and controls, such as the amount of movement, could be identified in this study. The possibility that differences in epileptogenic zone or the use of antiepileptic drugs contribute to the non-localized abnormalities cannot be ruled out.

\section{Interpretation of altered network topology}

In normal brain networks, high clustering coefficients ( $C$ and $\gamma$ ) and local efficiency ( $\left.E_{\text {local }}\right)$ are parameters which reflect a high local specialization (segregation) of information processing. Contrarily, low path length ( $L$ and $\lambda)$ and high global efficiency ( $\left.E_{\text {global }}\right)$ express a great ability to integrate information from the whole brain. Optimal brain networks possess both features with a balanced segregation and integration of information processing. ${ }^{18,34}$ In contrast, a decrease in clustering coefficient with a decrease in $\mathrm{IQ}$, as found in the patient population, is characteristic for more random networks and can be interpreted as a loss in network organization.

\section{Relation with intellectual decline}

In healthy controls, graph theoretical network parameters are related with level of intelligence $^{19}$ supporting the theory that cognitive processes depend on an optimal organization of segregation and integration. To our knowledge, this relation has not been investigated before in patients with epilepsy, who are prone to suffer from a range of cognitive deficits. It is an interesting observation that network topology changes in the presence of epilepsy, and that this alteration is associated with a decline of intellectual abilities.

\section{Clinical factors}

Of the clinical factors studied, only drug load was associated with network parameters. Antiepileptic drugs (AEDs) inhibit the spread of abnormal neuronal firing to distant sites, thereby suppressing the occurrence of clinical seizures. ${ }^{35}$ As AEDs might also have a more generalized suppressive effect, ${ }^{35}$ an alteration of neuroexcitability may affect local and global efficiency parameters such as found in the present study. There is too much overlap in AEDs used (Table 7.1) to provide any information on the effect of specific AEDs on network topological parameters, which 
would be of high interest for future studies. In an epilepsy population, it is complicated to disentangle the effects of AED use and epilepsy per se on network parameters. It would therefore be interesting to investigate whether AED use in other patient populations than those with epilepsy (e.g. in patients with migraine or neuropathic pain) is also associated with change of network parameters.

Although no other clinical factor could be identified to influence the network characteristics, this does not imply that no relation exists. For example, decreased local clustering and small-worldness within the epileptogenic temporal lobe has been associated with longer duration of temporal lobe epilepsy. ${ }^{36}$ Moreover, clinical epilepsy factors cannot be viewed as totally independent factors. For example, drug load is likely to increase when patients do not achieve seizure control. In that case, higher drug load can be a marker for a more severe form of epilepsy. Maybe it is not the seizures as such that disturb the cerebral networks, but simply the epilepsy itself. This could imply that there is a shared mechanism leading to both epilepsy and a disruption of cerebral networks that is associated with cognitive decline.

\section{Future perspectives}

Network parameters may obtain a role in identifying patients at risk for developing cognitive problems. If clinical factors can be identified, this can be beneficial in decision making: for example, patients with advantageous network organization may need less stringent seizure control than patients with disadvantageous network organization. Additionally, network topology might be a more sensitive marker for disease progression: if network parameters change before intellectual decline can be measured (normally clinically relevant decline can be observed only after intervals of several years), this may also call for more strict treatment of seizures, and/or earlier referral for epilepsy surgery. Finally, network topological characteristics might be of value in predicting cognitive outcome after epilepsy surgery. 


\section{Appendix 7.1. Premorbid FSIQ estimate calculation}

Based on the OPIE-3(4ST) from the Oklahoma Premorbid Intelligence Estimate for the WAIS-III Calculation Worksheet. ${ }^{21}$

Premorbid $\mathrm{FSIQ}=35.348+.368 *($ Vocabulary raw score $)+.682 *($ Information raw score $)+.987 *$ (Matrix Reasoning raw score $)+.737 *$ (Picture Completion raw score $)+$ $.175 *($ Age $)+.656 *($ Education code $)+.578 *($ Ethnicity code $)$.

Coding variables:

Age - in years

Education $-1=0-8$ years; $2=9-11$ years; $3=12$ years; $4=13-15$ years; $5=16+$ years

Ethnicity -1 = African-American; 2 = Hispanic; 3 = Other; 4 = Caucasian.

Note that all participants were Caucasian. 


\section{Appendix 7.2 Methods}

\section{Parcellation scheme}

The final brain parcellation into 892 regions was derived from the AAL template, consisting of 90 regions. We started by dividing each AAL region into two more or less equally sized regions by principal component analysis. The first principal component (a 3D vector), together with the center of gravity of the voxels within the region (a 3D point), defines a plane in 3D space which divides the region into two subregions according to the maximum spatial variance of the region (e.g. a "stretched" region will be divided along its main longitudinal axis).

Regions were subsequently subdivided with the criterion that a division must not yield a subregion with a size smaller than 125 voxels. The final result was a parcellation of 892 regions with comparable sizes (of at least 125 voxels).

\section{Sparsity}

Functional connection strengths between two areas $i$ and $j$ were obtained by calculating the Pearson correlation coefficients between the filtered time series of those regions. A connection matrix $A$ was formed by calculating correlation coefficients between all pairs of brain regions. The connection matrix $A$ is a numerical representation of a graph, which is an abstract data structure, consisting of nodes connected by edges. In the graph, a node is related to a brain region and is equal to a row or column from the connection matrix. An edge $e_{i, j}$ in the graph is a connection between brain areas $i$ and $j$, provided that $A_{i, j}>0$.

The brain graph of each individual was thresholded to create graphs with an equal number of nodes and edges across subjects. ${ }^{25}$ This was achieved by selecting the $T_{k}$ strongest connections (connections with the highest correlation coefficient and removing all other connections. The threshold value $T_{k}$ was expressed as a sparsity value relating the number of edges maintained in the network to the total number of edges possible $\left(N^{2}-N\right)$, where $N$ is the number of nodes (brain regions) in the network. ${ }^{26}$ Let $T_{k}$ be the number of edges maintained in the network, then the sparsity is defined as:

$$
\text { Sparsity }=\left(N^{2}-N-T_{k}\right) /\left(N^{2}-N\right)
$$

Theoretically, sparsity can range between 0 (a fully connected graph) and 1 (no edges). A low sparsity value (many connections in the network) implies that connections with a low correlation coefficient are also allowed to exist in the network, which might yield some false positive connections. With a high sparsity value, only edges with the highest correlation coefficients (i.e. strongest connections) remain. As 
a consequence, edges with intermediate correlation coefficients might be removed albeit being biologically plausible.

\section{Network metrics}

The degree $k$ of node $i$ is the number of connections to other areas:

$$
k_{i}=\sum_{j=1, j \neq i}^{N} A_{i, j},
$$

where $N$ is the total number of nodes (i.e. considered brain regions) in the graph.

The graph theoretical metrics characteristic path length $(L)$ and cluster coefficient $(C)$ as well as local and global efficiency were calculated to perform analysis on the constructed brain graphs.

Characteristic path length is defined as the average geodesic distance, in number of edges, connecting any two nodes in the graph:

$$
L=\frac{1}{N(N-1)} \sum_{i, j \in G, i \neq j} d_{i, j},
$$

Where $a_{i, j}$ is the length of the shortest path between nodes $i$ and $j$. The characteristic path length is a measure of how well connected a network is. Small characteristic path length indicates an average short distance between any two nodes, i.e. they can be reached through a small number of steps.

The cluster coefficient is defined as the number of actual edges connecting the neighbours of a node divided by the maximum number of edges possible between neighbouring nodes,

$$
C=\frac{1}{N} \sum_{i} \frac{\sum_{j, m} A_{i, j} A_{j, m} A_{m, i}}{k_{i}\left(k_{i}-1\right)} .
$$

The cluster coefficient of a network is a measure of how many local clusters exist in the network. A high cluster coefficient indicates that the neighbours of a node are often also directly connected to each other, i.e. they form a cluster. Parameters related to characteristic path length are global efficiency $\left(E_{\text {global }}\right)$ and local efficency $\left(E_{\text {local }}\right) . E_{\text {global }}$ is defined as the average inverse shortest path length, $E_{\text {local }}$ is defined as 
the mean of the global efficiencies of subgraphs consisting of the immediate neighbours of a particular node. These parameters may be meaningfully computed on disconnected graphs and are a superior measure of integration according to some authors. $^{26}$

\section{Random networks}

To be able to determine whether a network has small-world properties, the values of $L$ and $C$ must be scaled to values from generated random networks. ${ }^{24}$ Small world networks are characterized by having $L$ close to random: $\lambda=L / L_{\text {random }} \approx 1$, but with $C$ higher than random: $\gamma=C / C_{\text {random }}>1$. The value $\sigma=\gamma / \lambda$ can be used to signify the 'small-worldness' of a network and is typically larger than 1 for small-world networks. $^{37}$

Random networks were generated by considering each existing edge in the original network between the nodes $i$ and $j, e_{i, j}$ and connecting it to another randomly chosen node $j_{2}$, with the condition that $e_{i, j 2}$ was not present in the original network. ${ }^{38}$ This process ensures that the node degree and node distribution of the random network is similar to the original network. Network parameters were calculated with routines from the brain connectivity toolbox. ${ }^{39}$ 


\section{References}

1. Fisher RS, Vickrey BG, Gibson P, et al. The impact of epilepsy from the patient's perspective I. Descriptions and subjective perceptions. Epilepsy Res 2000;41:39-51.

2. Helmstaedter $C$, Kurthen M. Memory and epilepsy: characteristics, course, and influence of drugs and surgery. Curr Opin Neurol 2001;14:211-6.

3. Vlooswijk MC, Jansen JFA, Majoie HJM, et al. Functional connectivity and language impairment in cryptogenic localization-related epilepsy. Neurology 2010;75:395-402.

4. Oyegbile TO, Dow C, Jones J, et al. The nature and course of neuropsychological morbidity in chronic temporal lobe epilepsy. Neurology 2004;62:1736-42.

5. Jokeit H, Kramer G, Ebner A. Do antiepileptic drugs accelerate forgetting? Epilepsy Behav 2005;6: 430-2.

6. Thompson PJ, Duncan JS. Cognitive decline in severe intractable epilepsy. Epilepsia 2005;46:1780-7.

7. Cheung MC, Chan AS, Chan YL, Lam JM, Lam W. Effects of illness duration on memory processing of patients with temporal lobe epilepsy. Epilepsia 2006;47:1320-8.

8. Meletti S, Benuzzi F, Rubboli G, et al. Impaired facial emotion recognition in early-onset right mesial temporal lobe epilepsy. Neurology 2003;60:426-31.

9. Powell HW, Richardson MP, Symms MR, et al. Reorganization of verbal and nonverbal memory in temporal lobe epilepsy due to unilateral hippocampal sclerosis. Epilepsia 2007;48:1512-25.

10. Dupont S, Samson Y, Van de Moortele PF, et al. Delayed verbal memory retrieval: a functional MRI study in epileptic patients with structural lesions of the left medial temporal lobe. Neuroimage 2001;14:995-1003.

11. Weber B, Wellmer J, Schur S, et al. Presurgical language $\mathrm{fMRI}$ in patients with drug-resistant epilepsy: effects of task performance. Epilepsia 2006;47:880-6.

12. Vlooswijk MC, Jansen JF, de Krom MC, et al. Functional MRI in chronic epilepsy: associations with cognitive impairment. Lancet Neurol 2010;9:1018-27.

13. Waites $A B$, Briellmann RS, Saling MM, Abbott DF, Jackson GD. Functional connectivity networks are disrupted in left temporal lobe epilepsy. Ann Neurol 2006;59:335-43.

14. Addis DR, Moscovitch M, McAndrews MP. Consequences of hippocampal damage across the autobiographical memory network in left temporal lobe epilepsy. Brain 2007;130:2327-42.

15. Bettus G, Guedj E, Joyeux F, et al. Decreased basal fMRI functional connectivity in epileptogenic networks and contralateral compensatory mechanisms. Hum Brain Mapp 2009;30:1580-91.

16. Zhang Z, Lu G, Zhong $Y$, et al. Impaired attention network in temporal lobe epilepsy: a resting fMRI study. Neurosci Lett 2009;458:97-101.

17. Stam CJ, Reijneveld JC. Graph theoretical analysis of complex networks in the brain. Nonlinear Biomed Phys 2007;1:3.

18. Bullmore E, Sporns O. Complex brain networks: graph theoretical analysis of structural and functional systems. Nat Rev Neurosci 2009;10:186-98.

19. van den Heuvel MP, Stam CJ, Kahn RS, Hulshoff Pol HE. Efficiency of functional brain networks and intellectual performance. J Neurosci 2009;29:7619-24.

20. Wechsler D. Manual for the Wechsler Adult Intelligence Scale-III. San Antonio: The Psychological Corporation, 1997.

21. Schoenberg MR, Scott JG, Duff K, Adams RL. Estimation of WAIS-III intelligence from combined performance and demographic variables: development of the OPIE-3. Clin Neuropsychol 2002;16: 426-37.

22. Van Dijk KR, Hedden T, Venkataraman A, Evans KC, Lazar SW, Buckner RL. Intrinsic functional connectivity as a tool for human connectomics: theory, properties, and optimization. J Neurophysiol 2010;103:297-321.

23. Bassett DS, Bullmore E. Small-world brain networks. Neuroscientist 2006;12:512-23.

24. Watts DJ, Strogatz SH. Collective dynamics of 'small-world' networks. Nature 1998;393:440-2.

25. Stam CJ, Jones BF, Nolte G, Breakspear M, Scheltens P. Small-world networks and functional connectivity in Alzheimer's disease. Cereb Cortex 2007;17:92-9.

26. Achard S, Bullmore E. Efficiency and cost of economical brain functional networks. PLoS Comput Biol 2007;3:e17. 
27. Baron RM, Kenny DA. The moderator-mediator variable distinction in social psychological research: conceptual, strategic, and statistical considerations. J Pers Soc Psychol 1986;51:1173-82.

28. Liu Y, Liang M, Zhou Y, et al. Disrupted small-world networks in schizophrenia. Brain 2008;131: 945-61.

29. Liao W, Zhang Z, Pan Z, et al. Altered functional connectivity and small-world in mesial temporal lobe epilepsy. PLoS One;5:e8525.

30. Hermann B, Seidenberg M, Sears L, et al. Cerebellar atrophy in temporal lobe epilepsy affects procedural memory. Neurology 2004;63:2129-31.

31. Oyegbile TO, Bhattacharya A, Seidenberg M, Hermann BP. Quantitative MRI biomarkers of cognitive morbidity in temporal lobe epilepsy. Epilepsia 2006;47:143-52.

32. Focke NK, Yogarajah M, Bonelli SB, Bartlett PA, Symms MR, Duncan JS. Voxel-based diffusion tensor imaging in patients with mesial temporal lobe epilepsy and hippocampal sclerosis. Neuroimage 2008;40:728-37.

33. Bernhardt BC, Bernasconi N, Concha L, Bernasconi A. Cortical thickness analysis in temporal lobe epilepsy: reproducibility and relation to outcome. Neurology 2010;74:1776-84.

34. Sporns O, Chialvo DR, Kaiser M, Hilgetag CC. Organization, development and function of complex brain networks. Trends Cogn Sci 2004;8:418-25.

35. Rogawski MA, Loscher W. The neurobiology of antiepileptic drugs. Nat Rev Neurosci 2004;5:553-64.

36. van Dellen E, Douw L, Baayen JC, et al. Long-term effects of temporal lobe epilepsy on local neural networks: a graph theoretical analysis of corticography recordings. PLoS One 2009;4:e8081.

37. Humphries MD, Gurney K, Prescott TJ. The brainstem reticular formation is a small-world, not scalefree, network. Proc Biol Sci. 2006;273:503-11.

38. Maslov S, Sneppen K. Specificity and stability in topology of protein networks. Science. 2002;296: 910-3.

39. Rubinov $\mathrm{M}$, Sporns O. Complex network measures of brain connectivity: Uses and interpretations. Neuroimage. Neuroimage. 2010;52:1059-69. 



\section{Chapter 8}

General discussion 
136 Chapter 8 


\section{Rationale}

In daily practice, patients with epilepsy often report cognitive problems. ${ }^{1}$ Sometimes these are specific cognitive problems such as memory difficulties. These can often be related to underlying lesions. More frequently there are global problems such as attention problems, slowing of thought or even (mild) cognitive deterioration. Some describe seizure-related transient declines, occasionally even after a single seizure; others attribute their attention deficits to the use of antiepileptic drugs. The epilepsy syndrome may also be a crucial factor with more risk for localization-related epilepsies than idiopathic generalized epilepsies. ${ }^{2}$ Although extensive research has been done to unravel the connection of clinical epilepsy parameters with the occurrence of cognitive deficits, no clear-cut explanation or prognosis can be provided to the individual patient with non-symptomatic epilepsy.

The need for researchers to understand the cerebral processes responsible for the development of such cognitive problems was the basis for the research project described in this thesis. Since functional magnetic resonance imaging (fMRI) seems highly suitable to investigate functional processes such as cognition, its possibilities to study cognitive dysfunction in non-symptomatic or cryptogenic localization-related epilepsy were explored extensively.

\section{Approach}

First, an explorative study with 16 patients with epilepsy was performed. The patients had heterogeneous etiologies, but inclusion was based on the occurrence of secondarily generalized tonic-clonic seizures. This study aimed to explore the relationship of total number of secondarily generalized seizures with intellectual levels and $\mathrm{fMRI}$ activation patterns. The total number of secondarily generalized seizures is recognized as a risk factor for cognitive deterioration.

For the main study, it was decided to include only patients with cryptogenic localization-related epilepsies, to obtain a more homogeneous population and minimize the influence of structural cerebral abnormalities on the development of cognitive dysfunction. For the same reason, patients who had experienced a status epilepticus were excluded as well.

The participants underwent extensive examinations with acquisition of anatomic T1weighted MR images, four cognitive $\mathrm{fMRI}$ paradigms, diffusion-weighted MRI, and T2 relaxometry. A complete Wechsler Adult Intelligence Scale third edition (WAIS-III) was performed as well as additional neuropsychological tests for reading speed, memory, information processing speed, and handedness. 
In total, 56 patients and 27 healthy controls consented to participate in this project. For the separate analyses, data of fewer subjects were presented, since not all participants were able to undergo all subtests, or unexpected structural abnormalities on the 3.0 Tesla MRI images required exclusion from the analyses. Data analysis and presentation was divided according to different cognitive domains: memory, language, and global intelligence and intellectual decline.

\section{Cognitive deficits}

On all cognitive domains assessed with the extensive neuropsychological test battery - language, memory, information processing speed, general intelligence-, the patient group performed worse compared to the healthy control group. None of the separate domains was evidently more disturbed. As was expected, not all patients appeared evidently cognitively impaired, whereas a small subgroup exhibited obvious cognitive deficits.

\section{Effect of clinical epilepsy factors}

A wide variety of clinical factors was collected for all patients. In all analyses, the role of the number of secondarily generalized and partial seizures was assessed, as well as antiepileptic drug load, epilepsy duration, age at onset of seizures and site of seizure onset. In the first explorative study, a correlation of high number of generalized seizures with low intellectual level and with a change of $\mathrm{fMRI}$ activation patterns could be demonstrated. In the main study, this effect was lost due to the change of population. In this group of patients with cryptogenic epilepsy no major influence of the other clinical factors on the fMRI results could be demonstrated either. However, for the graph theoretical network analysis only, a correlation of antiepileptic drug use and less efficacious network parameters was observed.

Most of the clinical epilepsy factors are highly related and entangled which complicates straightforward investigation of the role of each individual factor. For example, high seizure frequency is often accompanied by higher drug load. Earlier age at onset is probably related with a more severe form of epilepsy, with higher seizure frequency. The fact that clinical factors were of no or minor influence in this patient population, does not imply that they play no role at all, but there role is subdued. Probably they act as a mediator or catalyst to the observed changes in neuronal networks. 


\section{Neuronal correlate of cognitive decline: from structural abnormalities to functional activation patterns and functional networks}

\section{Structural abnormalities}

This study aimed to explore the cerebral processes accompanying cognitive deficits in a population with cryptogenic localization-related epilepsy. By definition of the inclusion, no major effect of structural abnormalities on the most important outcome measures was expected. Hippocampal volumetry based on manual delineation on T1weighted images and comparison of total intracranial volume did not reveal significant differences between patients and controls. Furthermore, no relation with cognition was observed. This confirms the assumption that structural abnormalities as measurable with this MRI technique - do not underlie the observed cognitive differences between patients and controls in this particular population.

\section{Functional connectivity within predefined networks}

From a review of the currently available literature on $\mathrm{fMRI}$ activation studies focusing on cognition in localization-related epilepsy, the following can be concluded: (1) studies focusing on functional activation patterns could not identify one single overlapping key cerebral region to be involved in major cognitive processes such as memory and language, and (2) most of these studies describe a shift of activation. It is likely that the cerebral processes associated with cognitive dysfunction in epilepsy engage cerebral networks rather than isolated cerebral regions.

This was precisely what was observed in our study. With the first analyses of the fMRI data from the main study, the primary expectation was to find differences in activation patterns per se. However, activation patterns on all studied language and memory processing fMRI paradigms did not differ significantly between patients and controls according to whole brain activation analysis.

On the contrary, additional analyses assessing functional connectivity demonstrated relevant outcomes. For language processing, functional connectivity measures in the prefrontal and frontotemporal regions were lower in the patient group as compared to controls. For both memory and language processing, functional connectivity values in the patient group correlated with neuropsychological subtests for the respective cognitive domains. That is, lower functional connectivity is associated with worse neuropsychological performance.

For both cognitive processes, network functionality was investigated by comparing functional connectivity values, i.e. a measure expressing signal-time course similarity 
expressed by the correlation coefficient, for a set of predefined regions. These regions were selected based on their activation during the particular fMRI tasks.

It can be hypothesized that the outcomes in the language study were more pronounced due to the difference between the cognitive processes involved. Both language and (working) memory processes are engaged in most continuous daily cognitive processes, therefore theoretically requiring a thoroughly linked network. ${ }^{3}$ Especially the word generation task revealed the strongest effects, possibly due to the relatively higher (expressive) cognitive demand for this task as compared to other language and memory tasks.

Interestingly, for both the functional memory and language study it was observed that the fMRI paradigms engaging the prefrontal networks (word generation and Sternberg (verbal working memory)) revealed more pronounced differences between patients and controls than those involving the frontotemporal networks (text reading and picture encoding). This might be due to the fact that the prefrontal cortex and its inherent high number of connections are more prone to impairment than the temporal cortex in chronic epilepsy. Furthermore, it is acknowledged that it is more straightforward to activate the prefrontal regions than the temporal regions with the currently available fMRI paradigms. In part this may also be explained by technical issues with temporal brain regions being more prone to suffer from loss of BOLDsignal than prefrontal regions.

\section{Graph theoretical network analysis}

Graph theoretical network analysis offers the advantage over analysis of predefined networks that it investigates the topology of the whole brain network. ${ }^{4}$ Characteristics of a so-called 'small-world' network - which is acknowledged to be the most efficient network organization for all kinds of networks, including cerebral networks - include widespread local clustering in combination with some long-distance connections for rapid information transfer throughout the whole network. ${ }^{4}$ By its approach, this methodology does not rely on spatial overlap of functional activation or focal connection abnormalities, but assesses the whole brain functional connectivity.

The results in this study population point in the same direction as the outcomes of the functional network analyses with predefined regions of interest: whole-brain network topology is disturbed (less efficient) in patients with epilepsy. More remarkably, it was demonstrated that disturbance of graph theoretical network parameters was associated with lower intelligence levels and even with intellectual decline. 


\section{Localization-related epilepsy?}

If cognitive deficits in localization-related epilepsy were straightforward and directly linked to the anatomic site where seizures initiate, one would expect disturbance of memory functions in temporal lobe epilepsy, and deficits in executive functioning in frontal lobe epilepsy. Things are obviously more complicated. First, it has long become clear that cognitive processes are not confined to isolated cerebral regions and engage larger cerebral networks. Second, the results of this thesis demonstrate that functional abnormalities are diffuse and not constrained to the region of seizure onset.

The underlying mechanisms for these widespread abnormalities remain uncertain. The clinical factors assessed in these studies appeared not or were of minor influence on the functional abnormalities. A variety of factors may be involved: repetitive seizure spread, antiepileptic drugs, or focal interictal discharges may have a more distal effect. ${ }^{5}$ Alternatively, there may be pathological processes occurring before the onset of epilepsy that induce both the extensive functional abnormalities and the seizure disorders. ${ }^{6}$

\section{Clinical implications and future perspectives}

At present, the available $\mathrm{fMRI}$ techniques to measure functional connectivity values or determine single graph theoretical network parameters do not seem ready for interpretation for individual clinical patients. Currently, cognitive consequences of epilepsy can only be detected with neuropsychological assessment in a stage where the patient is already experiencing problems in daily life. Based on clinical variables, clinicians are unable to predict which patients are going to develop cognitive dysfunction. Ideally, one would like to perform MR imaging in combination with network analysis to provide a prognosis based on the neurological substrate to determine the individual patient's vulnerabilitiy for (further) cognitive decline.

The results presented in this thesis demonstrate that the mechanisms underlying or at least associated with cognitive dysfunction in cryptogenic localization-related epilepsies are neither based on structural abnormalities, nor on localized functional dysfunction as measured with functional MRI. The essential neuronal correlate rather appears to be a disruption of larger networks. Graph theoretical analysis of fMRI data seems especially suited to explore the role of whole-cerebrum networks in the development of cognitive deficits. Potentially this opens a window towards early detection of neuronal changes that increase the risk for the development of cognitive impairment. Early detection of patients at risk (compared to neuropsychological 
assessment of patients with existing cognitive impairments) may be valuable, especially in chronic epilepsy.

Research in the field of functional connectivity analysis has only recently started and techniques are rapidly evolving. Important issues to resolve include the definition of the ideal analyzing settings for functional MRI data and functional connectivity, and if possible, of standardized pre- and post-processing protocols. Important subjects are for example preference for resting-state versus task-related data, cut-off values for graph theoretical network parameters, and the influence of higher magnetic field strengths. In line with current functional connectivity studies, research is now focusing on the structural correlates of functional connectivity disturbances applying diffusiontensor imaging with visualization of fiber tracts and measurement of graph theoretical network parameters as well.

Many unanswered questions remain. For functional networks it is known that they can change in the course of epilepsy. Therefore it is important to detect the clinical factors affecting the network parameters negatively. One option, not explored in our study, is that frequent interictal epileptiform EEG discharges interfere with neuronal organization and lead to the observed disturbed networks. Conversely, it would be interesting to investigate whether neurocognitive training improves network parameters and thereby reduces the risks of cognitive dysfunction. If a window of opportunity could be identified during which the prognosis of cognitive dysfunction may be altered positively, and if we have more information on the interaction between clinical factors and neuronal disorganization, this would affect medical decision making. Longitudinal studies are needed to investigate these issues and provide a broader context for the findings presented in this thesis. Ideally, large cohorts would be followed even before onset of epilepsy, yet this is practically impossible. Research should therefore start at onset of epilepsy, and patients should be followed over large time periods. Eventually, identification of those patients at risk for developing cognitive decline based on the neurological substrate (e.g. including MRI network analysis) might lead to earlier therapeutic interventions and hopefully to a better neurocognitive outcome. 


\section{References}

1. Baker GA, Jacoby A, Buck D, Stalgis C, Monnet D. Quality of life of people with epilepsy: a European study. Epilepsia. 1997;38:353-62.

2. Elger CE, Helmstaedter C, Kurthen M. Chronic epilepsy and cognition. Lancet Neurol. 2004;3:663-72.

3. Raichle ME, MacLeod AM, Snyder AZ, Powers WJ, Gusnard DA, Shulman GL. A default mode of brain function. Proc Natl Acad Sci U S A. 2001;98:676-82.

4. Stam CJ, Reijneveld JC. Graph theoretical analysis of complex networks in the brain. Nonlinear biomedical physics. 2007;1:3.

5. Cole AJ. Is epilepsy a progressive disease? The neurobiological consequences of epilepsy. Epilepsia. 2000;41 Suppl 2:S13-22.

6. Taylor J, Kolamunnage-Dona R, Marson AG, Smith PE, Aldenkamp AP, Baker GA. Patients with epilepsy: Cognitively compromised before the start of antiepileptic drug treatment? Epilepsia. 2010;51:48-56. 

Summary 


\section{Summary}

The cognitive comorbidity often encountered in patients with localization-related epilepsy has long been studied by focusing on clinical factors that could underlie its development. Yet, an explanatory model for the cognitive problems could not be derived from such studies. The need for researchers and clinicians to understand the cerebral processes responsible for the development of cognitive problems in these patients, was the basis for the research project described in this thesis. The possibilities to study cognitive dysfunction in cryptogenic localization-related epilepsy with functional MRI (fMRI) and MR volumetry were explored extensively.

In chapter 2 results of previous $\mathrm{FMRI}$ studies in relation with cognitive functioning in patients with epilepsy are presented and interpreted. It is extremely difficult to compare individual fMRI studies because they differ on a variety of important aspects, such as the included patient population, the applied cognitive paradigms, and the fMRI data analysis techniques. The results of most studies though can be summarized in two possible processes: (1) there is a decreased activation in those regions normally activated in healthy controls, which is associated with cognitive dysfunction, or (2) there is a shift of activation from the normally activated regions to other cerebral regions. This shift of activation is usually considered as a dysfunctional process, i.e. associated with cognitive impairment.

Given that (1) those studies focusing on functional activation patterns could not identify one single key cerebral region to be involved in major cognitive processes such as memory and language, and (2) most of these studies describe a shift of activation, it follows that the cerebral processes associated with cognitive dysfunction in epilepsy engage larger cerebral networks rather than isolated cerebral regions.

In the second part of chapter 2, currently applied analysis techniques that address network functionality were discussed. These techniques all offer advantages and disadvantages, and the applicability in the investigation of cognitive problems in epilepsy is still being further explored.

In chapter 3, the results of the exploratory study are presented. This study was set out to investigate the effects of number of life-time secondarily generalized tonic-clonic seizures, one of the hallmark clinical factors, on cognitive function. fMRI activation patterns were analyzed in a rather global manner, focusing on relative activation levels in prefrontal and frontotemporal areas. This revealed a relative shift of activation from the frontotemporal to prefrontal brain regions in patients with higher number of life-time generalized seizures, as well as lower intelligence scores. The study design, the small number of patients, and the heterogeneity of epilepsy 
syndromes demand careful interpretation of these results, and a causal relationship between these three parameters cannot be concluded.

For the subsequent studies a new patient group was investigated, consisting only of patients with a non-symptomatic (cryptogenic) epilepsy.

Our goal in chapter 4 was to develop a practical, reproducible, and reliable protocol for the determination of hippocampal volumes by manual delineation. Guidelines and recommendations for image acquisition, manual delineation, delineation software, volume calculation and reliability measures were provided. It was demonstrated that the application of this protocol in our patient population resulted in reliable hippocampal volumes. In contrast to current practice, there was no additional value for correction for total intracranial volume. Although manual delineation remains very time-consuming, there is no (semi) automated delineation method that gives comparable reliable results. The protocol developed in chapter 4 was applied in chapter $\mathbf{5}$ to investigate hippocampal volumes in relation with memory assessment and $\mathrm{fMRI}$ of memory functions.

Both memory (chapter 5), as well as language functions (chapter 6), were evidently impaired in the patient group as compared to controls, although in general this group was characterized by milder cognitive impairment when compared to the former study. Activation patterns during fMRI tasks for memory and language processing did not differ significantly between both groups. For memory in particular, hippocampal volumes could not explain the cognitive differences. For both cognitive processes, network functionality was investigated by comparing functional connectivity values, i.e. a measure expressing signal-time course correlation, for a set of predefined regions. These regions were selected based on their activation during the particular fMRI tasks.

In the memory study (chapter 5), functional connectivity values did not differ between patients and controls. However, when focusing on the patient group, a correlation between a neuropsychological subtest for transient working memory and strength of functional connectivity within the selected prefrontal network was observed. That is, higher functional connectivity values correlated with better cognitive performance.

For fMRI of language processing (chapter 6), similar and even more pronounced results were obtained. Functional connectivity values in the prefrontal and frontotemporal regions were lower in the patient group as compared to controls. Functional connectivity values, especially within the prefrontal network, also correlated with language function. This effect increased when concentrating on those patients with worst performance on the neuropsychological language tests. 
In contrast to the approach in chapters $\mathbf{5}$ and $\mathbf{6}$ to study predefined functional networks including the regions recognized to participate in specific cognitive functions, graph theoretical network analysis offers the opportunity to investigate the topology of the whole-brain network. Characteristics of a so-called 'small-world' network - which is acknowledged to be a highly efficient network organization for all kinds of networks, including cerebral networks - include a high amount of clustering in combination with long-distance connections which enable efficient information transfer throughout the whole network. In chapter 7, graph theoretical network parameters were calculated for patients and controls, and correlated with a measure for intellectual decline. Chronic epilepsy interfered with normal small-world characteristics as were observed in healthy controls, with patients displaying both less clustering (a measure for segregation) and a lower path length (a measure for integration). It could be demonstrated that the severity of intellectual decline was associated with more disturbed local segregation. Although patients' networks still fulfill the criteria for a small-world network, a deviation towards more random networks, especially in those with more pronounced intellectual decline, was observed.

In chapter $\mathbf{8}$ the implications and applicability of the main findings of this thesis were discussed, as well as the possibilities for future research. 

Samenvatting 
152 


\section{Samenvatting}

Onderzoek naar cognitieve comorbiditeit bij patiënten met lokalisatiegebonden epilepsie is lang gericht geweest op de klinische factoren die hieraan ten grondslag zouden kunnen liggen. Dit heeft echter niet geleid tot een verklarend model voor die cognitieve problemen. De behoefte van onderzoekers en clinici om de cerebrale processen te begrijpen die betrokken zijn bij cognitieve problemen vormde de basis voor het onderzoeksproject dat heeft geleid tot dit proefschrift. De mogelijkheden om cognitieve dysfunctie in cryptogene lokalisatiegebonden epilepsie te onderzoeken middels functionele MRI ( $\mathrm{FMRI}$ ) en MR volumetrie werden uitgebreid onderzocht.

In hoofdstuk $\mathbf{2}$ werden de resultaten gepresenteerd en geïnterpreteerd van voorgaande $\mathrm{fMRI}$ studies die zich richtten op cognitief functioneren bij mensen met epilepsie. Het is buitengewoon moeilijk om afzonderlijke fMRI studies met elkaar te vergelijken, aangezien ze in vele aspecten van elkaar verschillen, zoals de patiëntenpopulatie, de cognitieve fMRI-testen en de technieken om de fMRI resultaten te analyseren. De resultaten van de meeste studies zijn echter samen te vatten in twee mogelijke processen: (1) er is minder activatie in die gebieden die bij gezonde controlepersonen geactiveerd worden, iets watgeassocieerd is met cognitieve dysfunctie, of (2) er is verschuiving van activatie waarbij andere dan de normaal geactiveerde gebieden actief worden. Deze activatieverschuiving wordt meestal beschouwd als een dysfunctioneel proces, d.w.z. geassocieerd met cognitieve stoornissen.

Op basis van de observaties dat (1) de studies die zich richten op functionele activatiepatronen niet één enkel hersengebied kunnen identificeren dat betrokken is bij de belangrijkste cognitieve processen zoals geheugen en taal, en dat (2) de meeste studies een activatieverschuiving beschrijven, kan worden opgemaakt dat niet de geïsoleerde hersengebieden, maar juist de grotere hersennetwerken betrokken moeten zijn bij die hersenprocessen die geassocieerd zijn met cognitieve stoornissen bij epilepsie.

In het tweede deel van hoofdstuk 2 werden huidige technieken voor analyse van netwerk functionaliteit besproken. De verschillende technieken hebben alle zo hun voor- en nadelen en de toepasbaarheid in het onderzoek naar cognitieve problemen bij epilepsie wordt momenteel nog nader onderzocht.

In hoofdstuk 3 werden de resultaten van het pilot onderzoek gepresenteerd. Deze was opgezet om de invloed van het aantal doorgemaakte secundair gegeneraliseerde epileptische aanvallen - een van de belangrijkste klinische factoren - op fMRI activatiepatronen en intelligentie te exploreren. De fMRI data werden geanalyseerd met de nadruk op relatieve activatieniveaus in prefrontale en frontotemporale 
gebieden. Hierbij werd een relatieve activatieverschuiving van de frontotemporale naar de prefrontale hersengebieden gezien bij patiënten met een hoog aantal doorgemaakte gegeneraliseerde aanvallen. Deze patiënten hadden ook lagere intelligentiescores. De studie-opzet, het kleine aantal patiënten en de heterogeniteit van epilepsiesyndromen vereisen een voorzichtige interpretatie van deze resultaten en een oorzakelijk verband tussen de drie parameters (aantal aanvallen, fMRI activatiepatroon en intelligentie) werd dan ook niet eenduidig aangetoond.

Voor de navolgende studies werd een nieuwe patiëntengroep onderzocht die uitsluitend bestond uit patiënten met niet-symptomatische (cryptogene) epilepsie.

Het doel van hoofdstuk 4 was de ontwikkeling van een praktisch, reproduceerbaar en betrouwbaar protocol om het hippocampale volume te bepalen middels manueel intekenen van MRI beelden. Richtlijnen en aanbevelingen voor beeldadquisitie, manueel intekenen, software, volumeberekening en betrouwbaarheidsmaten worden gegeven. Toepassing van dit protocol bleek tot betrouwbare hippocampale volumes te leiden in onze patiëntenpopulatie. In tegenstelling tot de gangbare praktijk was correctie voor totaal intracranieel volume niet van toegevoegde waarde. Hoewel manueel intekenen tijdrovend blijft, is er tot op heden geen (semi) geautomatiseerde methode die vergelijkbare resultaten geeft. Het protocol dat is beschreven in hoofdstuk 4 werd toegepast in hoofdstuk 5 om hippocampale volumes en de relatie met testresultaten voor geheugen en fMRI van geheugenfuncties te bestuderen.

Zowel het geheugen (hoofdstuk 5) als taalfuncties (hoofdstuk 6) waren duidelijk gestoord in onze patiëntengroep in vergelijking met de controlepersonen. De activatiepatronen tijdens $\mathrm{fMRI}$ taken voor geheugen en taal verschilden niet tussen patiënten en controles. Variaties in hippocampale volume konden de geheugenverschillen niet verklaren. Voor zowel geheugen als taal werd de netwerkfunctie bestudeerd door functionele connectiviteitswaarden - een maat voor correlaties van het signaalverloop over de tijd- tussen de groepen te vergelijken binnen een aantal hersengebieden. Deze gebieden waren geselecteerd op basis van hun activatie tijdens de desbetreffende cognitieve fMRI taken.

In de geheugenstudie (hoofdstuk 5) verschilden de functionele connectiviteitswaarden niet tussen patiënten en controles. Bij nader onderzoek van de patiëntengroep werd een verband gevonden tussen de neuropsychologische subtesten voor het vluchtige werkgeheugen en de functionele connectiviteitssterkte binnen het geselecteerde prefrontale netwerk. Een hogere functionele connectiviteit was gecorreleerd met een betere cognitieve prestatie.

In de fMRI studie naar taalprocessen (hoofdstuk 6) werden vergelijkbare resultaten gezien, maar deze waren nog meer uitgesproken. Functionele connectiviteitswaarden 
in de prefontale en frontotemporale gebieden waren lager in de patiëntengroep dan in de controlegroep. De functionele connectiviteitswaarden, vooral die binnen het prefrontale netwerk, correleerden ook met taalfunctie. Dit effect was het sterkst in die groep patiënten met de slechtste prestaties op de neuropsychologische taaltesten.

In tegenstelling tot de benadering in hoofdstukken 5 en 6 waarbij vooraf geselecteerde functionele netwerken werden onderzocht, biedt graaftheoretische netwerk analyse' de kans om de opbouw van het gehele breinnetwerk te bestuderen. Karakteristiek voor een zogenaamd 'small-world' netwerk - dat wordt beschouwd als de meest efficiente netwerkorganisatie voor allerlei netwerken, waaronder ook het brein - is uitgebreide lokale clustering in combinatie met enkele langeafstandsverbindingen voor snelle informatie-overdracht door het hele netwerk. In hoofdstuk 7 werden de 'graaftheoretische netwerk' parameters berekend voor patiënten en controles. Deze parameters correleerden met een maat voor intellectuele achteruitgang. Chronische epilepsie interfereert met normale 'smallworld' kenmerken zoals die in gezonde controles worden gezien, waarbij patiënten zowel minder clustering (een maat voor segregatie) als een kortere padlengte (een maat voor integratie) vertoonden. De ernst van intellectuele achteruitgang bleek geassocieerd met een gestoorde lokale segregatie. Hoewel de hersennetwerken van patiënten nog steeds voldoen aan de criteria voor een 'small-world' netwerk, wordt een afwijking richting een meer ongestructureerd opbouw van het netwerk gezien, met name bij die patiënten met een meer uitgesproken intellectuele achteruitgang.

In hoofdstuk 8 worden de betekenis en toepasbaarheid van de belangrijkste bevindingen van dit proefschrift besproken, evenals de mogelijkheden voor toekomstig wetenschappelijk onderzoek. 



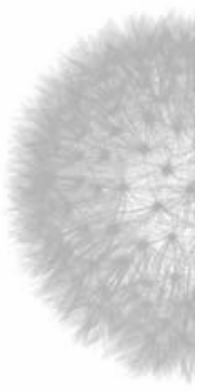

Dankwoord 
158 


\section{Dankwoord}

Het zit erop. Zes jaar geleden ging ik in zee met een enthousiaste groep mensen. In deze periode vorderde het onderzoek - mede door de combinatie met de klinische opleiding en gezinsuitbreiding - met hollen en stilstaan. Dankzij de tomeloze inzet van mijn collega's die het onderzoek draaiende hielden wanneer ik daar de tijd niet voor had, is het ons gelukt om de klus te klaren. Mijn dank hiervoor is groot.

Allereerst een woord van dank voor mijn promotieteam.

Prof. dr. A.P. Aldenkamp, beste Bert. Altijd enthousiast en optimistisch. Jij ziet door de bomen het bos en hebt duidelijk voor ogen waar het onderzoek naartoe moet, daar heb ik bewondering voor. Ik heb veel van je geleerd over de wetenschappelijke wereld. Dankzij jouw inspanningen kreeg ik tijd en ruimte voor onderzoek en schrijven. En jouw inzicht in de juiste thema's op de juiste tijd en plaats heeft ons een mooie publicatie opgeleverd!

Prof. dr. J.E. Wildberger. Sinds uw komst naar Maastricht is de aandacht voor het neuroradiologisch wetenschappelijk onderzoek binnen de afdeling radiologie verder toegenomen. Deze goede samenwerking tussen onze afdelingen is uitermate belangrijk voor het slagen van zo'n multidisciplinair onderzoek. Ik hoop dat we onze samenwerking de komende jaren kunnen voortzetten.

Dr. Ir. W.H. Backes, beste Walter. Jij hebt een buitengewoon grote bijdrage geleverd aan dit onderzoek. Altijd ongelooflijk snel en consciëntieus in je reacties op de vele versies van onze artikelen. En vaak genoeg heb je me uit de brand geholpen door zelf de kar te trekken. Je (onleesbare) schema's op het whiteboard schepten orde in de chaos in mijn hoofd. Wie weet wat we in de toekomst nog voor mooie projecten kunnen verzinnen en uitvoeren.

Dr. H.J.M. Majoie, beste Marian. Als studente mocht ik al met je meekijken in Kempenhaeghe en leerde je me de basis voor het opzetten van een database. Jij bent een echte workaholic en als ik dan eens trots was dat ik iets op zondagavond laat kon opsturen, had ik altijd binnen vijf minuten een superenthousiaste reactie! Het is duidelijk dat ook je patiënten je waarderen, aangezien ze voor jou graag de moeite namen om deel te nemen aan ons onderzoek. Ik zie uit naar onze samenwerking in de zorg voor patiënten met epilepsie.

Dr. J.F.A. Jansen, beste Jaap. Jij was 'de techneut' en ik 'de clinicus'. We zaten in hetzelfde schuitje en het klikte. Jij hebt alle patiënten gescand, meestal in de weekenden en avonduren, wat een klus. En dan nog het analyseren van de data waarvoor de computers soms het hele weekend moesten rekenen. Jij hebt me veel geleerd, zowel over de technieken - ondanks mijn terugkerende begripsstoornis bleef 
jij geduldig - als over het schrijven zelf. Samen schrijven was leerzaam en gezellig. Dankzij je keuze om na je eigen promotie nog een jaar te blijven, konden we de inclusie afronden. Ook tijdens je post-doc jaren in New York bleef je nauw betrokken bij ons onderzoek. Het is goed om je weer terug te hebben in Maastricht. Ik ben trots dat je - vier (!) jaar na je eigen promotie - nu als paranimf aan mijn zijde staat.

Dr. M.C.F.T.M. de Krom, beste Marc. Jij maakte me enthousiast voor het vak neurologie in het algemeen en voor de epileptologie in het bijzonder. Ik heb mooie herinneringen aan de epilepsiecongressen die we hebben bezocht, natuurlijk vooral aan het eerste congres in Parijs waar we elke dag een beter restaurant bezochten uit je Michelingids, samen met Ellen, Caroline en Joost. Ik heb er bewondering voor dat je na vele jaren ervaring nog steeds een grote interesse kon opbrengen voor je patiënten, assistenten en studenten.

Dr. P.A.M. Hofman, beste Paul. Bedankt voor je hulp bij het beoordelen van de structurele MRI-beelden en voor het aanleren van de beoordeling van de hippocampus. Dank ook voor het meelezen met de artikelen. Hopelijk kan ik nog eens in je voetsporen treden en op werkbezoek gaan in Melbourne.

Maarten Vaessen, mijn 'nieuwe Jaap'. Ik heb veel waardering voor jouw handigheid met netwerkanalyses en de toepassing ervan. Ik hoorde regelmatig mijn hersens kraken als we aan het brainstormen waren over ons small-world onderzoek! Je hebt bergen werk verzet om alle data te analyseren en het was een groot genoegen - niet alleen inhoudelijk, maar ook gewoon omdat we er lol in hadden - om het artikel samen te schrijven. Succes met de afronding van jouw promotietraject!

Cécile Crompvoets-Jeukens, dank voor je inspanningen om alle hippocampi in te tekenen. Het was misschien maar goed dat we vantevoren niet wisten hoeveel werk het zou zijn. Gelukkig konden we er wel om lachen en heeft het ook nog tot een mooie publicatie geleid. Dank ook voor je bijdrage aan het geheugenartikel.

Rianne Reijs, bedankt voor de database van de pilot study. En vooral ook dank voor je wijze lessen over promoveren en tips om staande te blijven!

Prof. dr. J.T. Wilmink, dank voor het beoordelen van de structurele MRI-beelden toen Paul Hofman in Australië zat. Tevens dank voor het voorzitten van de leescommissie.

De overige leden van de leescommissie, Prof. dr. P.A.J.M. Boon, Prof. dr. J.S.H. Vles en Dr. R.J. Van Oostenbrugge wil ik ook hartelijk danken voor de tijd en moeite die u hebt genomen om mijn proefschrift te beoordelen. 
Leonie Diepman, Annemarie Buermans, Caroline van der Linden en andere collega's van de gedragswetenschappelijke dienst in Kempenhaeghe: bedankt voor jullie hulp bij de logistiek en de neuropsychologische testen van alle patiënten. Ook de afdeling medische psychologie van het azM, met name Annelien Duits, bedankt voor de bijdrage aan het neuropsychologisch onderzoek.

Linda Linssen, dank voor je hulp bij de logistiek rond de afspraken met Marian en de geïncludeerde patiënten.

Collega-neurologen uit Kempenhaeghe en andere ziekenhuizen, dank voor de verwijzing van potentiële kandidaten voor de studie.

De patiënten en gezonde vrijwilligers verdienen een speciaal woord van dank. Het was een behoorlijke inspanning om voor de MRI naar Maastricht te komen - soms zelfs meerdere keren als de scanner defect was - en voor het overige onderzoek naar Kempenhaeghe. Dank ook voor het geduld om op de uitslagen te wachten!

Prof. P. Rieckmann and Prof. dr. M.H. De Baets, thank you for your help with the telencephalin analysis in the pilot study.

Tamara Martha en de medewerkers van het laboratorium in Kempenhaeghe, bedankt voor de analyse van het cytokinenprofiel voor de immunologische tak van het onderzoeksproject.

Koen Stakenborg, dank voor je hulp bij de analyse van de fMRI data.

Mijn collega's bij de neurologie. Jullie zag en zie ik soms vaker dan mijn familie of vrienden. Gelukkig is de samenwerking goed en gezellig. De neurologen wil ik bedanken voor de goede opleidingssfeer. Een aantal (oud-)collega's wil ik in het bijzonder noemen.

Ariane, Iris en Jordie, jullie waren en zijn de levende voorbeelden dat het kan: de combinatie van opleiding, onderzoek en moederschap. Jullie verdienen alledrie een medaille voor de prestaties die jullie hebben geleverd! We hebben grote lol gehad tijdens onze ladies nights. Hoewel jullie nu een stuk verder weg wonen, Jordie en Iris, blijft de interesse bestaan en dat waardeer ik. Ariane, wij wonen nog steeds bij elkaar om de hoek en kunnen eindeloos kletsen over allerlei belangrijke en onbelangrijke zaken, heerlijk. Ik vind het fantastisch dat je me wil bijstaan als paranimf.

De nieuwe ladies: Brigitte, Evelyn en Miesje. Ik voel me meer dan welkom bij jullie. Dank voor jullie openheid en vriendschap. 
De assistenten van het epilepsie-onderzoek: Hilde, Jordie, Kim en Nienke. Bedankt voor de gezelligheid tijdens congressen, symposia en etentjes. Succes met de afronding van jullie promotietrajecten.

Martien Limburg en Robert van Oostenbrugge, mijn opleiders, bedankt voor het faciliteren van de combinatie van wetenschappelijk onderzoek en opleiding. Ondanks de roerige tijden heb ik een gedegen opleiding genoten. Ik hoop er straks zelf ook een positieve bijdrage aan te leveren!

Kitty Verwoerdt, dank voor je eindeloze inspanningen om het rooster door te spitten op zoek naar mogelijkheden om onderzoeksvrij in te plannen!

De medewerkers in de kelder bij MRI-3, onder andere Marc, Jos, Max, Sanne, Doenja, Monique, Robbert, Robert-Jan, Eline, Eline, Vera, Suzanne, Frank, Karolien, Marlies, Bas, René, Kim. In zes jaar tijd mocht ik jullie af en toe een paar maanden versterken. Bedankt voor de moeiteloze integratie, de lunch, de vlaai, de praatjes bij de koffieautomaat, maar vooral ook voor alle hulp bij computer-, netwerk- en printproblemen (bedankt Marc!).

De geneesco-vrienden: Bregje, Sanne, Max, Wout, Leon, Marianne, Stance en Laurette. We zijn een zooitje ongeregeld maar ik zou onze weekenden - en de vriendschappen - voor geen goud willen missen.

Lieve Patrick en Cornelis, ik trad in jullie beider voetsporen, geografisch gezien dan. Geen Harvard, maar Maastricht en later als Erasmus-student naar Madrid. We zijn alledrie helemaal anders, maar door onze gezamenlijke achtergrond en de interesse in elkaar blijft de familieband sterk. Hopelijk lukt het om jullie prachtige families straks weer wat vaker te gaan zien!

Lieve papa en mama, dank voor alles wat jullie me hebben meegegeven in dit leven. $\mathrm{Nu}$ ik zelf een gezin heb, is de waardering alleen maar gegroeid. Van jullie brede interesses en maatschappelijke betrokkenheid kan ik nog wat leren! Dank voor de mogelijkheden om mij te ontwikkelen tot wie ik ben, dank voor de vrijheid om de wereld te verkennen en dank voor de liefde en interesse die ik dagelijks ervaar.

Liefste Alexander, jouw komst heeft me geleerd de zaken beter in perspectief te zien. En geloof het of niet, jij was een goede motivatie om dit proefschrift af te maken. Jij leert me genieten van het moment, van kleine mooie dingen. Ik houd van jou.

Oscar, vida de mi vida. Tú eres la base, el fundamento. Mi mejor amigo, mi apoyo, mi alegría. Gracias por estar a mi lado, siempre. Nuestro amor hace que todo vale la pena. Te amo. 


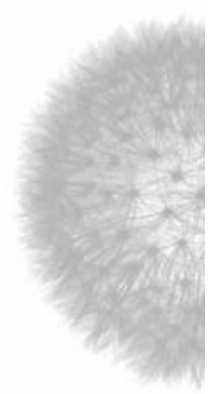

List of publications 
164 


\section{List of publications}

Vlooswijk MC, Vaessen MJ, Jansen JF, de Krom MC, Majoie HJ, Hofman PA, Aldenkamp AP, Backes WH. Loss of network efficiency associated with cognitive decline in chronic epilepsy. Neurology. 2011;77:938-944.

Vlooswijk MC, Klinkenberg S, Hageman G, Dellemijn PL, Roeleveld AB, Vles JS. [Epidural hematoma in children after falling from a relatively low height]. TNN. Accepted.

Vlooswijk MC, Jansen JF, Jeukens CR, Majoie HJ, Hofman PA, de Krom MC, Aldenkamp AP, Backes WH. Memory processes and prefrontal network dysfunction in cryptogenic epilepsy. Epilepsia 2011;52(8):1467-75.

Vlooswijk MC, Jansen JF, de Krom MC, Majoie HM, Hofman PA, Backes WH, Aldenkamp AP. Functional MRI in chronic epilepsy: associations with cognitive impairment. Lancet Neurol. 2010 Oct;9(10):1018-27.

Vlooswijk MC, Jansen JF, Majoie HJ, Hofman PA, de Krom MC, Aldenkamp AP, Backes $\mathrm{WH}$. Functional connectivity and language impairment in cryptogenic localizationrelated epilepsy. Neurology. 2010 Aug 3;75(5):395-402.

Jeukens CR, Vlooswijk MC, Majoie HJ, de Krom MC, Aldenkamp AP, Hofman PA, Jansen JF, Backes WH. Hippocampal MRI volumetry at 3 Tesla: reliability and practical guidance. Invest Radiol. 2009 Sep;44(9):509-17.

Jansen JF, Vlooswijk MC, Majoie HM, de Krom MC, Aldenkamp AP, Hofman PA, Backes WH. White matter lesions in patients with localization-related epilepsy. Invest Radiol. 2008 Aug;43(8):552-8.

Jansen JF, Vlooswijk MC, de Baets $\mathrm{MH}$, de Krom MC, Rieckmann P, Backes WH, Aldenkamp AP; SEGAED Study Group. Cognitive fMRI and soluble telencephalin assessment in patients with localization-related epilepsy. Acta Neurol Scand. 2008 Oct;118(4):232-9.

Vlooswijk MC, Jansen JF, Reijs RP, de Krom MC, Kooi ME, Majoie HJ, Hofman PA, Backes WH, Aldenkamp AP. Cognitive fMRI and neuropsychological assessment in patients with secondarily generalized seizures. Clin Neurol Neurosurg. 2008 May;110(5):441-50.

Vlooswijk MC, Majoie HJ, De Krom MC, Tan IY, Aldenkamp AP. SUDEP in the Netherlands: a retrospective study in a tertiary referral center. Seizure. 2007 Mar;16(2):153-9. 
Curriculum Vitae 
168 


\section{Curriculum Vitae}

Mariëlle Vlooswijk werd geboren op 4 augustus 1980 in Linschoten. In 1998 behaalde zij het VWO-diploma aan het Minkema Collega in Woerden. Aansluitend begon zij aan haar studie geneeskunde aan de Universiteit Maastricht. In het derde studiejaar volgde zij een semester aan de geneeskundefaculteit van de Universidad Complutense in Madrid. In 2001-2002 was zij lid van de opleidingscommissie van geneeskunde. In het vijfde studiejaar deed zij haar co-schappen in het Hospital Clínico San Carlos in Madrid. Daarnaast deed zij stages en co-schappen in Paramaribo (Suriname), Arequipa (zuid-Peru) en lquitos (Amazonegebied in Peru). Tijdens haar co-schap neurologie in het Maastricht Universitair Medisch Centrum (MUMC) werd zij enthousiast voor dit vak. Aan het einde van haar studie deed zij onder begeleiding van Prof. Dr. A. Aldenkamp, Dr. M. De Krom en Dr. M. Majoie onderzoek in het azM en epilepsiecentrum Kempenhaeghe naar het vóórkomen van acuut onverklaard overlijden bij epilepsie (SUDEP). Vanaf 2004 is zij werkzaam als arts-assistent bij de vakgroep neurologie in het azM alwaar zij vanaf maart 2005 in opleiding is tot neuroloog (opleiders Prof. Dr. M. Limburg, Dr. M. de Krom en sinds 2010 Dr. R. van Oostenbrugge). Tijdens deze opleiding startte zij met het onderzoek naar fMRIbevindingen bij cognitieve problemen in epilepsie dat heeft geresulteerd in dit proefschrift. Half september 2011 zal zij de opleiding neurologie afronden en per 1 november 2011 zet zij haar carrière voort als neuroloog in het MUMC.

Mariëlle Vlooswijk was born on August $4^{\text {th }} 1980$ in Linschoten, the Netherlands. In 1998, she finished her secondary school at the Minkema Collega in Woerden. Subsequently, she enrolled in her medical training at Maastricht University. In her third year of medical school she studied a semester at the Complutense University in Madrid. In 2000-2001 she was a member of the Programme Committee of the Medical Faculty. In her fifth year, she returned to Madrid for internships at the Hospital Clínico San Carlos. She also did internships in Paramaribo (Surinam), Arequipa (south-Peru) and Iquitos (Amazon region in Peru). Her internship at the department of neurology at the Maastricht University Medical Center (MUMC) was the basis of her enthusiasm for this specialty. At the end of her medical training, she studied the prevalence of sudden unexpected death in epilepsy (SUDEP) at the MUMC and epilepsy center Kempenhaeghe under supervision of Prof. Dr. A. Aldenkamp, Dr. M. de Krom, and Dr. M. Majoie. Afterwards, she started as a non-resident at the Department of Neurology in Maastricht, where she continued as a resident from March 2005 (Prof. Dr. M. Limburg, Dr. de Krom, and from 2010 Dr. R. van Oostenbrugge). During her residency, she started with the research on fMRI findings in cognitive impairment in epilepsy which has resulted in this thesis. She will complete her residency in September 2011 and continue her career as a neurologist at the MUMC. 
